\title{
Zinc Anode for Mild Aqueous Zinc-Ion Batteries: Challenges, Strategies, and Perspectives
}

Cite as

Nano-Micro Lett.

(2022) $14: 42$

Received: 14 October 2021

Jinzhang Yang ${ }^{1}$, Bosi Yin ${ }^{1}$, Ying Sun ${ }^{1}$, Hongge Pan ${ }^{2,3}$, Wenping Sun ${ }^{3}$, Baohua Jia ${ }^{4}$,

Accepted: 24 November 2021

Siwen Zhang ${ }^{\natural}$, Tianyi Ma ${ }^{4}$

Published online: 3 January 2022

(C) The Author(s) 2022

\section{HIGHLIGHTS}

- This article mainly summarizes the challenges and the latest research progress of highly reversible zinc anodes in mild aqueous zincion batteries and proposes corresponding recommendations.

ABSTRACT The rapid advance of mild aqueous zinc-ion batteries (ZIBs) is driving the development of the energy storage system market. But the thorny issues of $\mathrm{Zn}$ anodes, mainly including dendrite growth, hydrogen evolution, and corrosion, severely reduce the performance of ZIBs. To commercialize ZIBs, researchers must overcome formidable challenges. Research about mild aqueous ZIBs is still developing. Various technical and scientific obstacles to designing $\mathrm{Zn}$ anodes with high stripping efficiency and long cycling life have not been resolved. Moreover, the performance of $\mathrm{Zn}$ anodes is a complex scientific issue determined by various parameters, most of which are often ignored, failing to achieve the maximum performance of the cell. This review proposes a comprehensive overview of existing $\mathrm{Zn}$ anode issues and the corresponding strategies, frontiers, and development trends to deeply comprehend the essence and inner connection of degradation mechanism and performance. First, the formation mechanism of dendrite growth, hydrogen evolution, corrosion, and their influence on the anode are analyzed. Furthermore, various

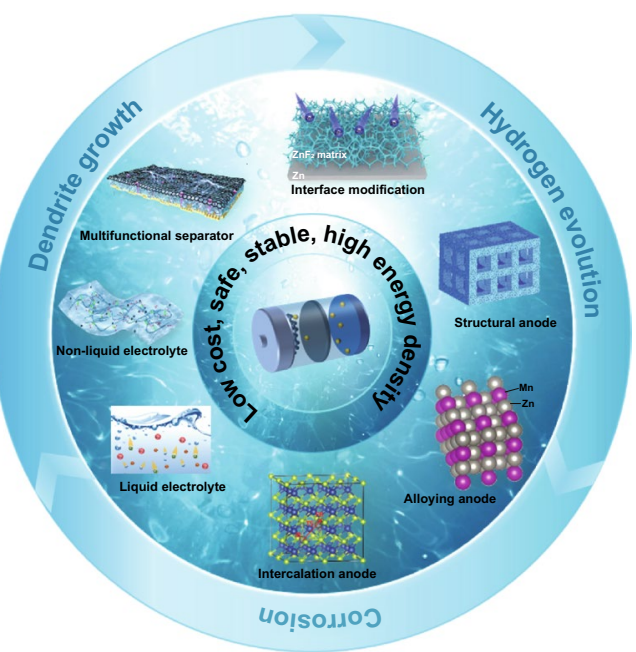
strategies for constructing stable $\mathrm{Zn}$ anodes are summarized and discussed in detail from multiple perspectives. These strategies are mainly divided into interface modification, structural anode, alloying anode, intercalation anode, liquid electrolyte, non-liquid electrolyte, separator design, and other strategies. Finally, research directions and prospects are put forward for $\mathrm{Zn}$ anodes. This contribution highlights the latest developments and provides new insights into the advanced $\mathrm{Zn}$ anode for future research.

KEYWORDS Zn-ion batteries; Zn metal anode; Dendrite; Hydrogen evolution; Corrosion

Siwen Zhang, zhangsiwen@lnu.edu.cn; Tianyi Ma, tianyima@swin.edu.au

1 Key Laboratory for Green Synthesis and Preparative Chemistry of Advanced Materials of Liaoning Province, Institute of Clean Energy Chemistry, College of Chemistry, Liaoning University, Shenyang 110036, People's Republic of China

2 Institute of Science and Technology for New Energy, Xi'an Technological University, Xi'an 710021, People's Republic of China

3 State Key Laboratory of Clean Energy Utilization, School of Materials Science and Engineering, Zhejiang University, Hangzhou 310027, People's Republic of China

4 Centre for Translational Atomaterials, Swinburne University of Technology, Hawthorn, VIC 3122, Australia 


\section{Introduction}

With the sharp increase in energy demand, in response to the global challenges of the increased depletion of traditional fossil energy and associated environmental issue [1-3], many countries and regions have increased their investment in renewable energy such as solar energy, wind energy, and hydropower energy [4-6]. Although there are low- or noncarbon clean energy sources, the generation and conversion of the renewable power supply systems are intermittent, unstable, and uncontrollable, which makes energy storage and transportation difficult $[7,8]$. Therefore, as a medium to regulate electricity output and improve the tolerance ability of the power grid to renewable energy, the development of ESS technology is highly essential to enable a clean energy transition [9]. Among the various ESSs, non-aqueous lithium-ion batteries (LIBs) are currently the most widely used rechargeable electrochemical devices $[10,11]$. However, owing to flammable organic electrolytes and highly reactive lithium substances, the increasing concerns about the potential safety issues hinder the application of LIBs on a large scale [12-14]. Besides, the high cost and also the low abundance of lithium resources on the earth limited the long-term development of LIBs $[15,16]$.

Compared with traditional organic electrolytes-based LIBs, aqueous metal-ion batteries have proved promising for large-scale energy storage since the adopted aqueous electrolyte possesses the characteristic of more safety, lower cost, easier processing, and higher ionic conductivity. Currently, various aqueous metal-ion batteries have been developed, such as zinc-ion batteries (ZIBs), sodium-ion batteries (SIBs), potassium-ion batteries (PIBs), aluminum-ion batteries (AIBs), magnesium-ion batteries (MIBs), and calcium-ion batteries (CIBs) [17]. Compared to other active metals, $\mathrm{Zn}$ metal can be directly used as an anode due to its proper redox potential (-0.76 V vs. standard hydrogen electrode (SHE)) and excellent $\mathrm{Zn} / \mathrm{Zn}^{2+}$ reversibility aqueous media. The high natural abundance (approximately 300 times higher than that of lithium) and good resistance to the environment allow $\mathrm{Zn}$ to be purchased and processed inexpensively. Very importantly, the $\mathrm{Zn}$ anode also has the inherent advantage of high theoretical capacity $\left(820 \mathrm{mAh} \mathrm{g}^{-1}\right.$ and $5854 \mathrm{mAh} \mathrm{cm}^{-3}$ ). Therefore, aqueous ZIBs have attracted sufficient attention $[18,19]$. According to the $\mathrm{pH}$ of electrolytes, aqueous ZIBs can generally be divided into alkaline ZIBs and mild (neutral or mildly acidic) ZIBs. The research of zinc-based batteries traces its history back to the nineteenth century. It was not until the 1980s that Yamamoto et al. first studied rechargeable $\mathrm{Zn}-\mathrm{MnO}_{2}$ batteries in $2 \mathrm{M} \mathrm{ZnSO}_{4}$ electrolyte, which created a precedent for the development of mild aqueous ZIBs $[20,21]$. It has been confirmed that the substitution of mild electrolyte for alkaline electrolyte can usually exhibit better reversibility due to many advantages, such as eliminating passivation and alleviating dendrite growth on the anode surface. Thus, these reports about mild aqueous ZIBs have emerged endlessly in recent years.

As an integral part of ZIBs, it is well known that anodes are particularly important to the performance and lifespan of batteries. However, although $\mathrm{Zn}$ anodes have inherent advantages, as mentioned above, there are also some thorny problems, which may be devastating to ZIBs. Besides, considerable research efforts have been devoted to the cathode side, such as manganese-based and vanadium-based materials [22, 23], while the research focus on the anode side is still in the primitive stage [24]. Inadequate exploration of $\mathrm{Zn}$ anodes leads to a vague understanding of $\mathrm{Zn}$ anode failure. It remains a challenge to solve the problems of $\mathrm{Zn}$ dendrite growth, hydrogen evolution, and corrosion on the $\mathrm{Zn}$ anode side in mild aqueous ZIBs. Based on the previous reports, dendrite growth significantly reduces $\mathrm{Zn}$ anode's capacity and Coulomb efficiency (CE). Large dendrites can even pierce the battery separator and cause a short circuit due to the high mechanical strength and Young's modulus of Zn. Besides, compared with the traditional alkaline electrolyte, the weakly acidic environment provides a stronger thermodynamic trend for hydrogen evolution. The irreversible consumption of electrolytes during hydrogen evolution and corrosion impairs the long-term cycling performance of ZIBs, and the generated hydrogen increases the risk of battery splitting and electrolyte leakage. Even worse, the mutual reinforcement among these issues causes further deterioration of anode performance [25]. Therefore, in-depth study and optimization of $\mathrm{Zn}$ anodes are necessary for ZIBs to move toward practical applications.

To construct highly stable $\mathrm{Zn}$ anodes, researchers have proposed various strategies in mild aqueous ZIBs. Considering that it is essential to improve the comprehension of anode challenges and grasp the direction of anode development via recognizing diverse strategies, a detailed summary is necessary. Some previous reviews generally divided these strategies into the following categories: anode matrix 
modification, electrolyte optimization, and separator design. However, with an in-depth study on $\mathrm{Zn}$ anodes, new discoveries and novel modification strategies continue to emerge, and the previous generalizations can no longer keep pace with the latest progress, especially unable to give in-depth and clear explanations for some basic issues. Therefore, this review summarizes recent research from a broad perspective, including more comprehensive basic knowledge and the latest work on $\mathrm{Zn}$ anodes. At the same time, based on a large number of published studies, this work elaborates some specific mechanisms in depth, which were mentioned in other reviews but failed to give a detailed explanation, such as how the following facts profoundly affect $\mathrm{Zn}$ deposition: the electronic conductivity and $\mathrm{Zn}$ affinity of modification layer, $\mathrm{Zn}$ alloying, and so on.

This review aims to provide a comprehensive summary of the recent development of $\mathrm{Zn}$ anode in mild aqueous $\mathrm{ZIBs}$ (as shown in Fig. 1). First, the main challenges, involving dendrite growth, hydrogen evolution, corrosion, and the interaction relationship between them, are systematically analyzed to identify $\mathrm{Zn}$ deposition behavior at $\mathrm{Zn}$ anode. Then, various latest strategies to enhance the anode performance are categorized and discussed in detail, including interface modification (redistribution of concentration field, redistribution of electric field, and regulation of surface binding energy),

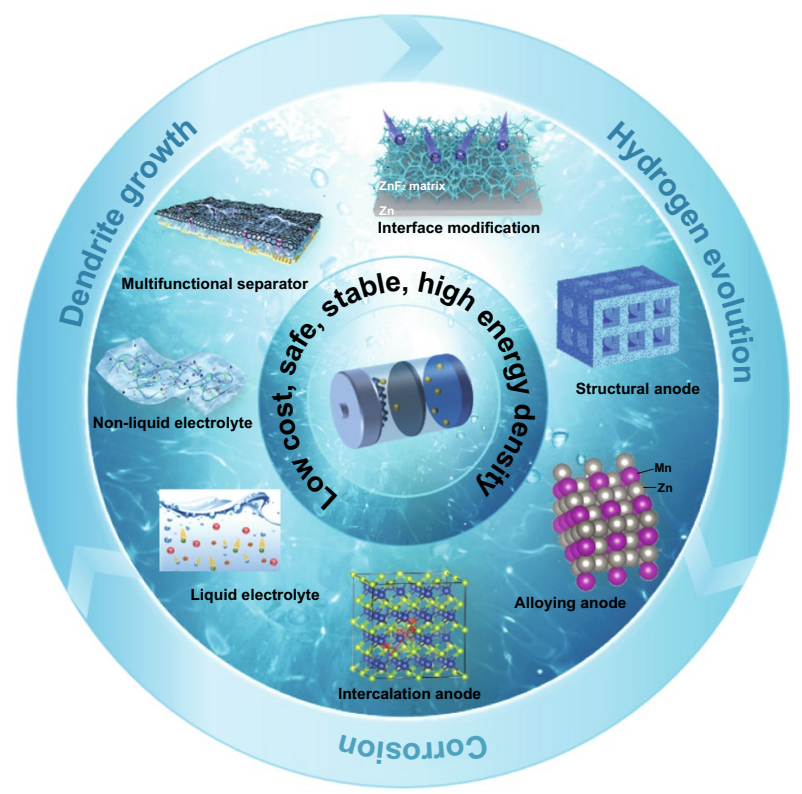

Fig. 1 Schematic of strategies to enhance the performance of $\mathrm{Zn}$ anodes for mild aqueous ZIBs structural anode, alloying anode, intercalation anode, liquid electrolyte (weakening of solvation effect, suppression of $2 \mathrm{D}$ diffusion, formation of electrostatic shielding layer, and formation of in situ solid electrolyte interphase (SEI) layer), non-liquid electrolyte (solid-state electrolyte, hydrogel electrolyte, and other non-liquid electrolytes), separator design, and other strategies. Finally, by analyzing the latest research achievement, Zn anode's remaining challenges and perspectives are proposed to produce more reliable aqueous ZIBs with rationally improved performance.

\section{Challenges of Reversible Zn Anode in Mild Aqueous ZIBs}

The possible reactions at the anode/electrolyte interface play significant roles during the energy storage and release of ZIBs. Generally, unlike alkaline systems with zincates as charge carriers, the mild aqueous ZIBs involve the reversible plating/stripping of $\mathrm{Zn}^{2+}$ ions on anode surface accompanying the charging/discharging operations. The reaction mechanism of $\mathrm{Zn}$ anode can be summarized as

Discharge process:

$\mathrm{Zn} \rightarrow \mathrm{Zn}^{2+}+2 \mathrm{e}^{-}$

Charge process:

$\mathrm{Zn}^{2+}+2 \mathrm{e}^{-} \rightarrow \mathrm{Zn}$

Simultaneously, given that $\mathrm{Zn}$ has a high electrochemical activity and thermodynamical instability in mild aqueous electrolytes, leading to side reactions, there are also anode-related disadvantages, such as dendrite growth, hydrogen evolution and corrosion (Fig. 2a), which will be analyzed as follows.

\subsection{Dendrite Growth}

Currently, it is generally accepted that $\mathrm{Zn}$ dendrite growth is the major problem in mild aqueous ZIBs. Zn dendrites affect battery performance in several ways. On the one hand, due to the loose structure, flake-like $\mathrm{Zn}$ dendrites easily fall off the electrode and form "dead Zn," thus reducing the CE and shortening the battery lifespan (Fig. 2b). On the other hand, the vertical growth of dendrites increases the thickness of the anode, and large dendrites may pierce the separator, 
(a)

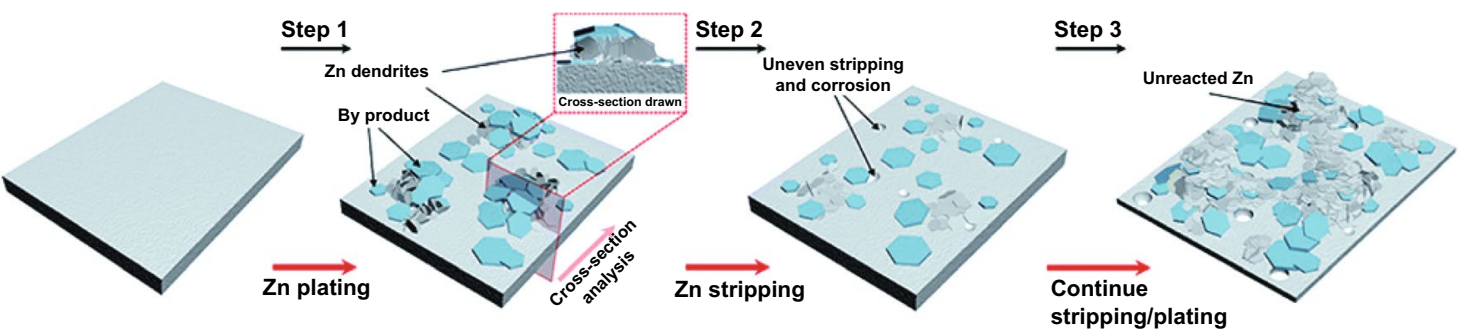

(b)

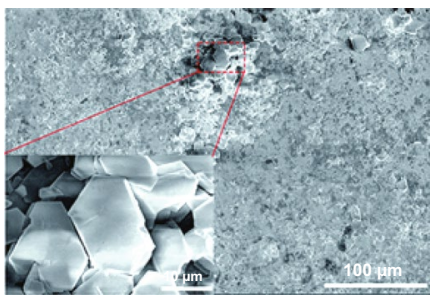

(c)

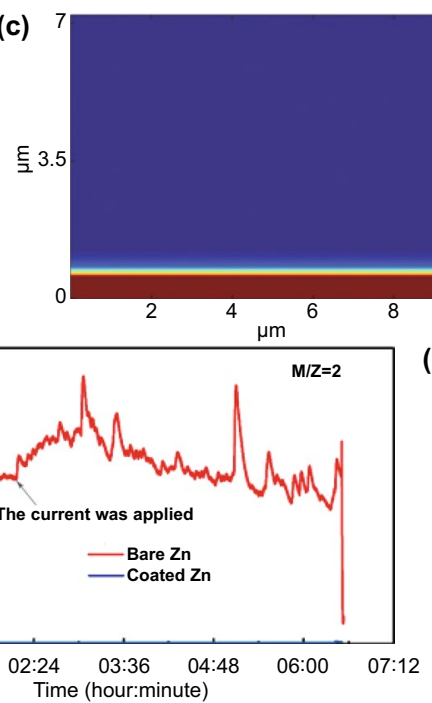

(d)
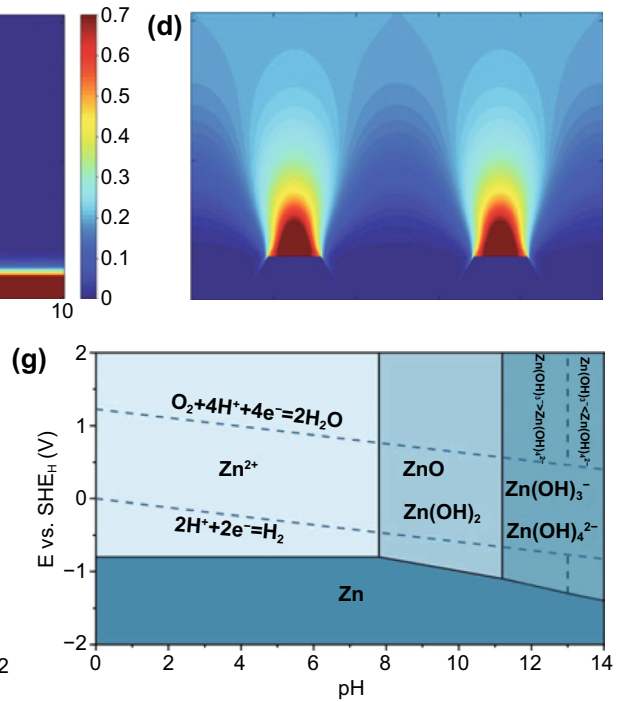

(h)

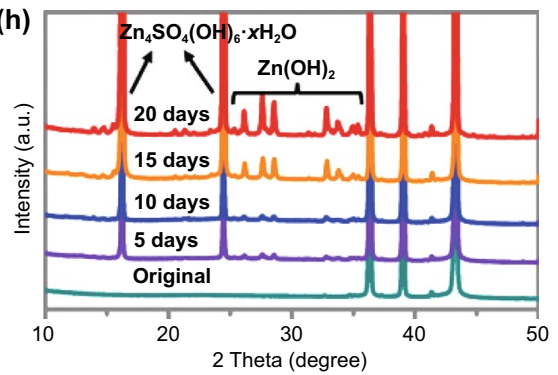

(i)

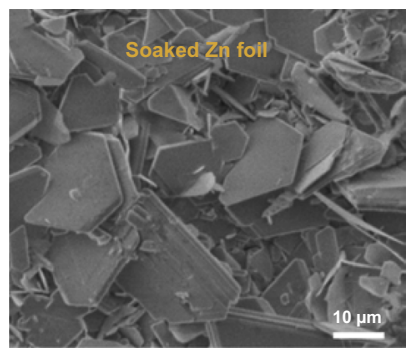

(j)

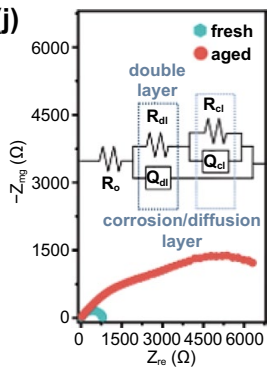

Fig. 2 a Schematic illustration of the formation of inactive Zn; b Top-view SEM image of the Zn electrode after short circuit. Inset: flake-like dendrites [137]. Copyright 2021, Royal Society of Chemistry. Simulation of the diffusion and distribution of Zn ions along the 2D surface of the electrode with the conditions of $\mathbf{c}$ a flat surface and $\mathbf{d} 2$ large dendritic seeds [29]. Copyright 2019, Wiley-VCH. e In situ optical microscope images of $\mathrm{H}_{2}$ gas evolution during the $\mathrm{Zn}$ electrodeposition process at $0.2 \mathrm{~mA} \mathrm{~cm}{ }^{-2}$ [42]. Copyright 2019, Elsevier. f Online DEMS data for symmetrical $\mathrm{Zn}$ batteries with the bare $\mathrm{Zn}$ in $2 \mathrm{M} \mathrm{ZnSO}_{4}$ electrolyte, reflecting the hydrogen evolution of the anode during rest and charging/discharging process [43]. Copyright 2019, Royal Society of Chemistry. g Pourbaix diagram of $\mathrm{ZnSO}_{4}-\mathrm{H}_{2} \mathrm{O}$ system at $25{ }^{\circ} \mathrm{C}$ [44]. Copyright 2021, American Chemical Society. $\mathbf{h}$ The in situ XRD patterns of bare $\mathrm{Zn}$ immersed in $2 \mathrm{M} \mathrm{ZnSO}_{4}$ electrolyte [48]. Copyright 2021, Wiley-VCH. i SEM image of $\mathrm{Zn}$ foil after soaking in $1 \mathrm{M} \mathrm{ZnSO}_{4}$ electrolyte for 7 days [41]. Copyright 2020, Wiley-VCH. $\mathbf{j}$ Nyquist plots of the fresh and aged $\mathrm{Zn}$ electrode. The inset shows the equivalent circuit [49]. Copyright 2021, Wiley-VCH

causing a short circuit in the batteries. Even worse, faulty batteries may trigger safety incidents such as explosions or fires.

As we all know, Zn dendrites are caused by uneven deposition during the charging process. Specifically, $\mathrm{Zn}^{2+}$ ions transfer to the anode surface under the dual effect of the electric field and concentration gradient at the beginning of $\mathrm{Zn}$ plating, followed by acquiring electrons and nucleating [26]. Theoretically, nucleation positions should be randomly distributed on an anode surface. But the electrode surface morphology is challenging to be infinitely smooth, and there is a certain degree of roughness. In particular, due to the "tip effect," compared to other locations, the protrusion with large curvature features higher surface charge density, which stimulates 
stronger local electric field intensity [27]. Furthermore, even on an infinitely smooth plane, the $\mathrm{Zn}$ nucleus formed earlier will act like a "protrusion" to affect the subsequent deposition of $\mathrm{Zn}^{2+}$ ions [28]. As a result, driven by the effect of electric field and concentration gradient, $\mathrm{Zn}^{2+}$ ions accelerate to accumulate and deposit on the tip (Fig. 2c, d) [29].

It can be known that many factors affect the growth of dendrites in mild aqueous ZIBs, and the connections between them are intricate. It has been proven that the $\mathrm{Zn}$ deposition process is affected by electrode polarization, especially concentration polarization. At a specific current density and electrode concentration, $\mathrm{Zn}^{2+}$ ions in the electrolyte continuously migrate to the anode reaction interface, where $\mathrm{Zn}^{2+}$ ions are consumed. Due to the limited migration speed, the subsequent $\mathrm{Zn}^{2+}$ ions cannot be supplemented to the reaction interface in time, resulting in a large concentration gradient perpendicular to the anode surface. The polarization caused by this concentration difference is the concentration polarization. The large concentration polarization is not conducive to the rapid transfer kinetics of $\mathrm{Zn}^{2+}$ ions. Besides, concentration polarization can lead to an increase in the overpotential on the anode surface. According to the ultra-thin electric double-layer model on the $\mathrm{Zn}$ surface, a large overpotential will seriously disturb the uniformity of the electric field, causes uneven $\mathrm{Zn}$ deposition, and destroys the stability and reversibility of the anode [20]. Therefore, the concentration polarization should be as small as possible. In addition, the current density has an important influence on the performance of the $\mathrm{Zn}$ anode, which can directly affect the $\mathrm{Zn}$ deposition rate. In the diffusion model (Eq. 3), the "Sand's time $\tau$ " is empirically related to the transfer properties of $\mathrm{Zn}^{2+}$ ions and electrons as Eq. 3 [30]:

$\tau=\pi D \frac{e C_{0}\left(\mu_{a}+\mu_{Z n^{2+}}\right)^{2}}{2 J \mu_{a}}$

where $\tau$ is the time when $\mathrm{Zn}$ dendrites start to grow, and $D$ is the diffusion coefficient. $e$ is the electronic charge. $C_{0}$ is the initial concentration of $\mathrm{Zn}$ salt. $\mu_{a}$ and $\mu_{\mathrm{Zn}^{2+}}$ are the anionic and $\mathrm{Zn}^{2+}$ mobility, respectively. $J$ is the effective electrode current density. The smaller effective electrode current density $(J)$ and larger $\mathrm{Zn}^{2+}$ mobility $\left(\mu_{\mathrm{Zn}^{2+}}\right)$ result in a larger Sand's time $(\tau)$, which indicates that the battery has a long lifespan before $\mathrm{Zn}$ dendrites grow. This can be explained by the fact that the small current density can lead to a more uniform local current density distribution and a smaller surface electric field distortion [31, 32]. Correspondingly, in a neutral/mildly acidic electrolyte, Yang et al. [29] observed that $\mathrm{Zn}$ dendrites gradually appeared and accelerated their growth with the increase in current density. It is proposed that a large current density will increase electrode polarization and cause uneven $\mathrm{Zn}$ deposition. On the contrary, a small current density is beneficial to alleviate the dendrite problem. In order to obtain a small current density, a reasonable reduction in the applied current and an increase in the specific surface area of the anode can be considered. At the same time, the capacity (the area capacity corresponding to the area of a symmetric battery or the mass load of the cathode material in an asymmetric battery) has also been found to be another critical factor affecting the growth of $\mathrm{Zn}$ dendrites. Large capacity will require more time to complete a single charge process, which leads to more severe dendrite formation [29]. Additionally, perfect metal manufacturing process and polished metal surface/edges can reduce anode defects [33]; the improved $\mathrm{Zn}$ affinity of the matrix can lower the nucleation energy barrier [34]; the high operating temperature can lead to increased diffusion coefficient, reduced concentration polarization, large nuclei size, low nucleation density, and compact growth of $\mathrm{Zn}$ metal $[35,36]$. An appropriately low $\mathrm{pH}$ of the electrolyte can enable metal $\mathrm{Zn}$ to deposit in a hexagonal structure, instead of the inclined pyramidal structure at high $\mathrm{pH}$ [37]. Appropriate external pressure can offset the stress response caused by the intrinsic strain triggered by $\mathrm{Zn}$ deposition $[38,39]$. By manipulating these factors, the problem of $\mathrm{Zn}$ dendrite growth can be alleviated. Based on the above analysis, the appearance of $\mathrm{Zn}$ dendrites in neutral or mildly acidic electrolytes largely depends on the battery configuration and charge/discharge protocol.

\subsection{Hydrogen Evolution}

In addition to $\mathrm{Zn}$ deposition, other species may be involved in side reactions on the anode, such as dissolved oxygen in the electrolyte and the electroactive materials of soluble cathodes. But the primary side reaction is the hydrogen evolution reaction (HER) caused by water (Fig. 2e, f) [40]. In fact, due to the influence of various factors such as temperature, applied voltage, electrode surface roughness, and electrolyte concentration, HER is a complicated process, which can be described as follows [41]:

Anode:

$\mathrm{Zn} \leftrightarrow \mathrm{Zn}^{2+}+2 e^{-}$ 
Cathode hydrogen evolution:

$2 \mathrm{H}_{2} \mathrm{O}+2 \mathrm{e}^{-} \leftrightarrow 2 \mathrm{OH}^{-}+\mathrm{H}_{2} \uparrow$

The hydrogen evolution at the anode occurs during the rest and operation of the battery [42, 43]. Given that the equilibrium potential of $\mathrm{Zn}^{2+} / \mathrm{Zn}(-0.76 \mathrm{~V}$ vs. SHE) is lower than that of $\mathrm{H}_{2} \mathrm{O} / \mathrm{H}_{2}(0 \mathrm{~V}$ vs. SHE) in the entire $\mathrm{pH}$ range (Fig. 2g), the coexistence of $\mathrm{Zn}$ and $\mathrm{H}_{2} \mathrm{O}$ is thermodynamically unstable, which means that the two will react spontaneously and release hydrogen [44]. During the plating process, there is a competitive reaction between metal deposition and hydrogen evolution; hydrogen evolution is theoretically preferred over $\mathrm{Zn}$ deposition. However, according to the Tafel equation, the $\mathrm{Zn}$ mental anode presents a high HER overpotential, which is not conducive to hydrogen evolution kinetics; thus, the rate of HER is limited to a certain degree [45].

The mild aqueous ZIBs suffer from HER, which can irreversibly consume electrodes and electrolytes, thereby reducing battery life and CE. Also, with the gradual accumulation of hydrogen, the battery expands due to the increase in internal pressure until it bursts, ultimately causing electrolyte leakage [46]. In addition, the continuous evolution of hydrogen will cause changes in the local $\mathrm{pH}$ of electrolytes (Eq. 5), which is related to the formation of corrosion, exacerbating the negative effect on the battery. It is still an urgent challenge to propose an ideal strategy to solve the hydrogen evolution problem based on the above issues.

\subsection{Corrosion}

Simultaneously, the corrosion phenomenon accompanying the hydrogen evolution has also attracted long-term attention. Corrosion in mild systems is inseparable from HER, so electrochemical corrosion is also a thorny problem. Specifically, many micro-galvanic cells are formed at the phase interface of $\mathrm{Zn}$ metal/electrolyte. $\mathrm{Zn}$ metal at the corrosion site is dissolved due to the loss of electrons, and $\mathrm{H}_{2} \mathrm{O}$ in the neutral solution obtains electrons to generate hydrogen and $\mathrm{OH}^{-}$[47]. It has been confirmed that the continuously accumulated $\mathrm{OH}^{-}$will further react with the electrolyte to form by-products on the anode surface. However, different types of electrolytes and operating environments may have different byproducts. As we all know, $\mathrm{Zn}(\mathrm{OH})_{2}$ and $\mathrm{Zn}_{4} \mathrm{SO}_{4}(\mathrm{OH})_{6} \cdot \mathrm{xH}_{2} \mathrm{O}$ with a hexagonal structure can be formed in $\mathrm{ZnSO}_{4}$ electrolyte (Fig. 2h), and the main reactions involved are as follows [48]:

$4 \mathrm{Zn}^{2+}+6 \mathrm{OH}^{-}+\mathrm{SO}_{4}^{2-}+x \mathrm{H}_{2} \mathrm{O} \leftrightarrow \mathrm{Zn}_{4} \mathrm{SO}_{4}(\mathrm{OH})_{6} \cdot x \mathrm{H}_{2} \mathrm{O}$

Similarly, other mild electrolytes also participate in $\mathrm{Zn}$ corrosion and form by-products, such as TFSI-based complexes which can be formed in $\mathrm{Zn}(\mathrm{TFSI})_{2}$ electrolyte. Unfortunately, due to the loose structure of these by-products, they cannot act as a SEI layer to escape from the corrosion reaction (Fig. 2i) [41]. Moreover, the increased interphase impedance caused by the by-product layer restricts the diffusion of electrons/ions, which triggers a high energy barrier for $\mathrm{Zn}$ deposition. It severely reduces the performance of the battery (Fig. 2j) [49].

It is worth noting that dendrite growth, hydrogen evolution, and corrosion are inseparable. The three promote each other. The loose and porous $\mathrm{Zn}$ dendrites increase the contact area between the electrode and electrolyte, which provides more reaction sites and reduces the current density to achieve a low overpotential, leading to accelerated hydrogen evolution and corrosion. The adhesion of hydrogen bubbles on the anode surface can hinder the nucleation of $\mathrm{Zn}$, resulting in an increased overpotential and uneven $\mathrm{Zn}$ deposition. At the same time, the accumulation of $\mathrm{OH}^{-}$anions caused by HER accelerates the corrosion process. The rough $\mathrm{Zn}$ surface triggered by corrosion may also further aggravate dendrite formation. The by-product layer with large curvature and irregularity also increases the contact area, accelerating the HER. Therefore, a specific anode modification strategy is usually beneficial to alleviate the three problems simultaneously.

\section{Design and Optimization of High-performance $\mathrm{Zn}$ Anode in Mild Aqueous ZIBs}

According to the above analysis, the anode problems, including dendrite growth, hydrogen evolution, and corrosion, hinder the commercialization of mild aqueous ZIBs. It is imperative to explore and develop efficient and stable $\mathrm{Zn}$ anode protection strategies. The mild aqueous ZIBs are advancing rapidly; especially in recent years, numerous novel and unique research ideas and achievements continue to emerge. This section summarizes and discusses recent developments from multiple perspectives, including interface modification, 
structural anode, alloying anode, intercalation anode, liquid electrolyte, non-liquid electrolyte, separator design, and other strategies.

\subsection{Interface Modification}

In the process of $\mathrm{Zn}^{2+}$ ion diffusion, reduction, nucleation, and $\mathrm{Zn}$ crystal growth, considering that dendrite growth and HER mainly occur at the anode/electrolyte interface, it seems a direct and effective strategy to construct a surface modification layer. The modified layer usually plays multiple roles, and this section focuses on redistribution of concentration field, redistribution of the electric field, and regulation of surface binding energy. These, respectively, directly manipulate the migration behavior of ions and molecules on the electrolyte side, the electron distribution on the anode side, and the nucleation barrier at the reaction interface to guide uniform $\mathrm{Zn}$ nucleation and growth and inhibit side reactions.

\subsubsection{Redistribution of Concentration Field}

The redistribution of concentration field mainly adjusts the spatial distribution and migration behavior of $\mathrm{Zn}^{2+}$ ions, $\mathrm{H}_{2} \mathrm{O}$ molecules, hydrated $\mathrm{Zn}^{2+}$ ions, and anions at the anode reaction interface, thereby improving subsequent $\mathrm{Zn}$ deposition and inhibiting side reactions. As mentioned earlier, restricting the migration behavior of $\mathrm{Zn}^{2+}$ ions during the deposition process has a profound impact on dendrite growth. Besides, it is already known that the participation of $\mathrm{H}_{2} \mathrm{O}$ molecules in the anode interface reaction will severely reduce the electrode performance. Specifically, in the electrolyte containing a large amount of free water, $\mathrm{Zn}^{2+}$ ion $(0.74 \AA)$ can be solvated to form a bulky hydrated structure $\left[\mathrm{Zn}\left(\mathrm{H}_{2} \mathrm{O}\right)_{6}\right]^{2+}(4.3 \AA)$, which is not conducive to the rapid ion transfer [50]. Moreover, $\mathrm{Zn}^{2+}$ ions surrounded by $\mathrm{H}_{2} \mathrm{O}$ molecules are difficult to effectively contact the reaction interface, causing a low electrochemical reaction activity, and $\left[\mathrm{Zn}\left(\mathrm{H}_{2} \mathrm{O}\right)_{6}\right]^{2+}$ must be desolvated to participate in the subsequent reaction. Thus, there is an additional demand for energy to overcome the strong interaction between $\mathrm{Zn}^{2+}$ ions and $\mathrm{H}_{2} \mathrm{O}$ solvation sheath. Even worse, the free $\mathrm{H}_{2} \mathrm{O}$ molecules transferred from the electrolyte or generated after the desolvation process may participate in side reactions, causing hydrogen evolution and corrosion [51]. Anions in the electrolyte are also related to anode performance. On the one hand, since anions will not be solvated, they have high ion mobility and contribute a lot to ionic conductivity, but this reduces the effective ion conductivity provided by $\mathrm{Zn}^{2+}$ ions. On the other hand, anions may participate in the formation of by-products [52]. Based on the above considerations, the regulation mechanisms of modified layers, involving inhibiting the $2 \mathrm{D}$ diffusion of $\mathrm{Zn}^{2+}$ ions, reducing concentration polarization, reducing the degree of hydration, and restricting $\mathrm{H}_{2} \mathrm{O}$ molecules and anions from entering the anode reaction interface, are conducive to achieving uniform and rapid $\mathrm{Zn}$ deposition without hydrogen evolution and corrosion. Therefore, designing a suitable interface modification layer to directly redistribute concentration field is a feasible strategy to enhance the performance of $\mathrm{Zn}$ anodes. Generally, a modified layer has multiple interrelated means to achieve these goals. Next, the regulation mechanism of these modified layers will be described separately from the mechanical guidance of confined channels, adsorption guidance of polar groups, and directional electric field guidance of aligned dipoles.

As a buffer layer that separates the active $\mathrm{Zn}$ from the bulk electrolyte, especially electronic insulation properties, the concentration field can generally be mechanically adjusted by constructing the confined channel, such as porous or layered structure. According to the size-selective exclusion effect, to enable the stable migration of $\mathrm{Zn}^{2+}$ ions and block other larger-sized molecules and ions, the confined channel structure should be well organized and have an appropriate channel size [53]. For instance, the Nafion-Zn-X modified layer formed by the complexation of Nafion and Zn-X zeolite (Fig. 3a) [54] or the hydrogen-substituted graphodiyne (HsGDY) layer constructed by cross-coupling of $\mathrm{C}_{12} \mathrm{H}_{6}$ monomer (Fig. 3b) [55] and their ion tunnels can only selectively transfer $\mathrm{Zn}^{2+}$ ions. In contrast, $\mathrm{H}_{2} \mathrm{O}$ molecules, hydrated $\mathrm{Zn}^{2+}$ ions, and $\mathrm{SO}_{4}{ }^{2-}$ ions with larger sizes cannot pass through the modified layer, so the effective mass transfer is significantly improved. Furthermore, the porous channel structure can serve as a physical barrier to inhibit the 2D diffusion of $\mathrm{Zn}^{2+}$ ions. Regardless of the initial uneven electric field on the anode surface, abundant sub-nanometer ion tunnels of HsGDY affect the migration path of $\mathrm{Zn}^{2+}$ ions (Fig. 3b): Since $\mathrm{Zn}^{2+}$ ions cannot move between tunnels, they can only be transferred along the tunnels and then nucleate and grow on the corresponding anode surface under the tunnels, rather than a thermodynamically 
(a) Surface of Nafion-Zn-X
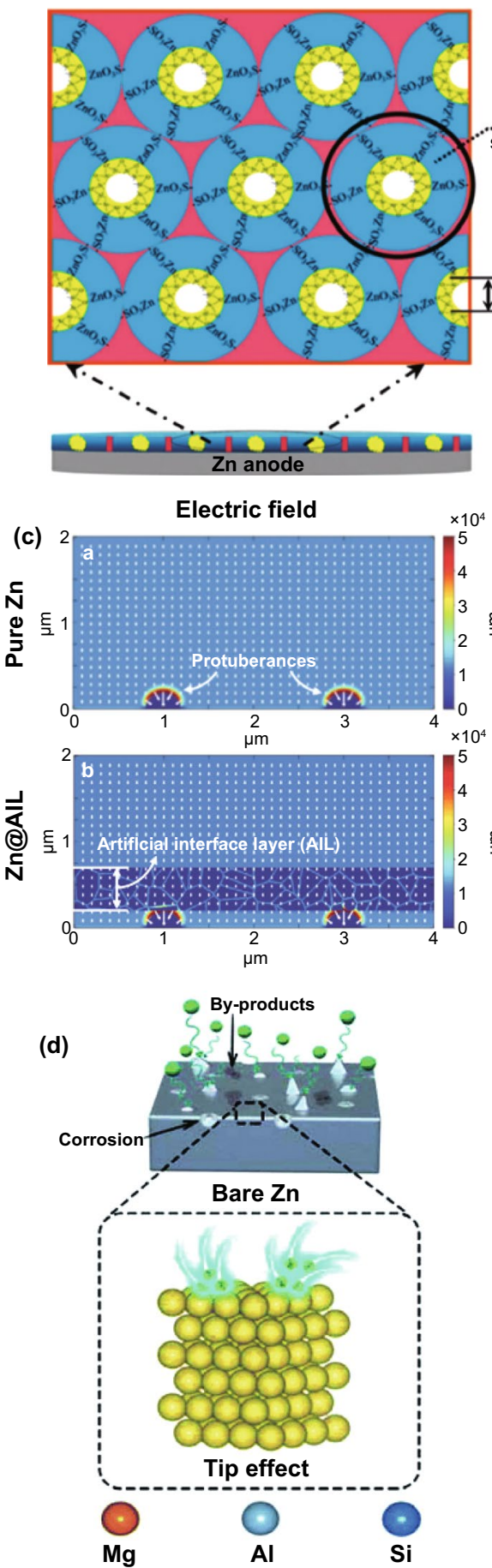
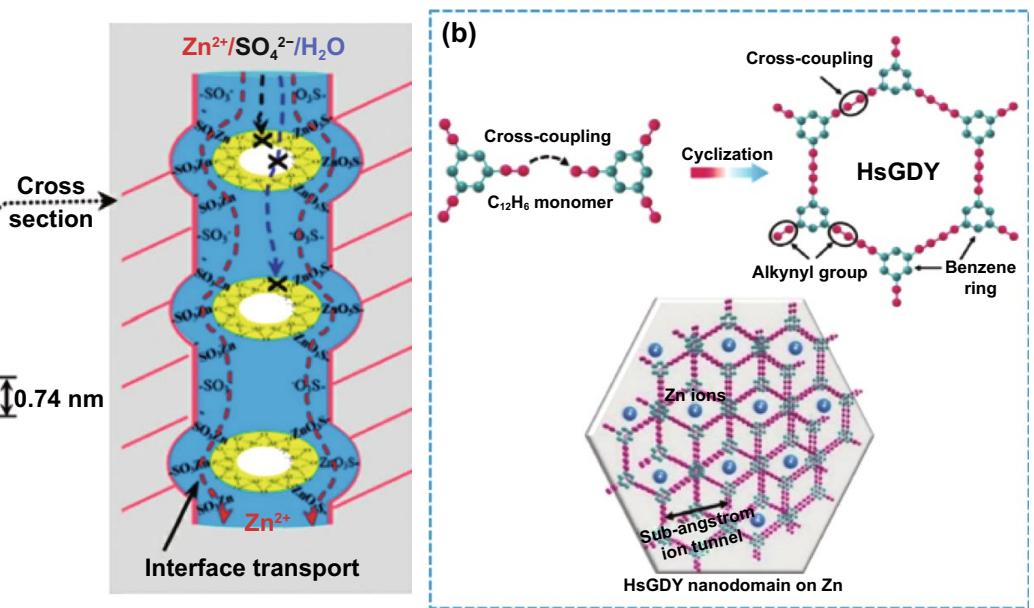

Concentration field
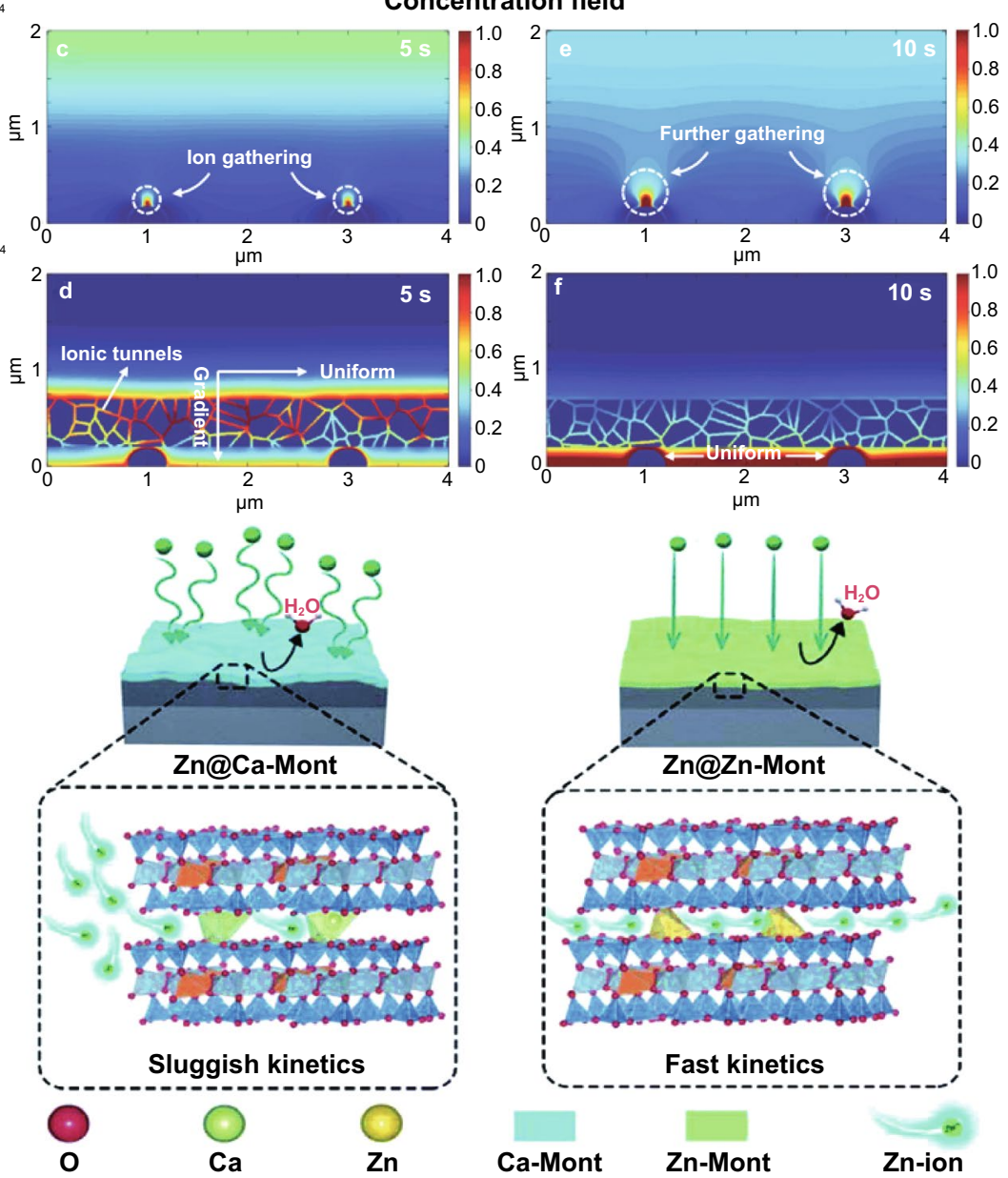

Fig. 3 a Ion transport mechanisms in Nafion-Zn-X protective layers [54]. Copyright 2020, Wiley-VCH. b Schematic illustration of the synthesis of HsGDY and the sub-ångström ion tunnel of HsGDY; $\mathbf{c}$ Electric and concentration field simulation with protuberances of bare $\mathrm{Zn}$ and coated Zn [55]. Copyright 2020, Wiley-VCH. d Schematic illustration of the Zn deposition process on bare Zn, Zn@Ca-Mont and Zn@ZnMont anodes [58]. Copyright 2021, Elsevier 
favorable adsorption site with a low energy barrier. The inhibited 2D diffusion of $\mathrm{Zn}^{2+}$ ions promotes the formation of a uniform $\mathrm{Zn}^{2+}$ ion concentration field along with the HsGDY-Zn interface. The simulation of the concentration field well demonstrated the ion redistribution effect of the ion tunnel (Fig. 3c). It has been proved that the channel structure's high porosity and small pore size are more conducive to accelerating the mass transfer capacity and forming a uniform $\mathrm{Zn}$ nucleus. The broadly and densely distributed pores ensure universal and uniform initial nucleation sites on the $\mathrm{Zn}$ surface. For some inorganic metal-based compounds, such as nanoporous $\mathrm{CaCO}_{3}$ [56], nanoporous $\mathrm{SiO}_{2}$ [56], kaolin [57], Zn-based montmorillonite [58, 59], and $\mathrm{Mg}-\mathrm{Al}$-layered double hydroxide (LDH) [60], they are also chemically inert and electronically insulating; therefore, the concentration field redistribution in these modified layers is mainly through their confined channel (Fig. 3d), thereby inhibiting dendrites, hydrogen evolution, and corrosion. Obviously, due to the rigid structure of these materials, the lack of mechanical flexibility is not conducive to adapting to changes in the volume of the negative electrode, resulting in limited enhancement of battery performance.

In addition to constructing intuitive confined channels to mechanically change the concentration field, many organic materials can directly and selectively manipulate the migration of $\mathrm{Zn}^{2+}$ ions to redistribute concentration field with the assistance of their specific polar groups (Fig. 4a), such as the amide group in polyamide (PA) [43], the poly(vinyl (a)

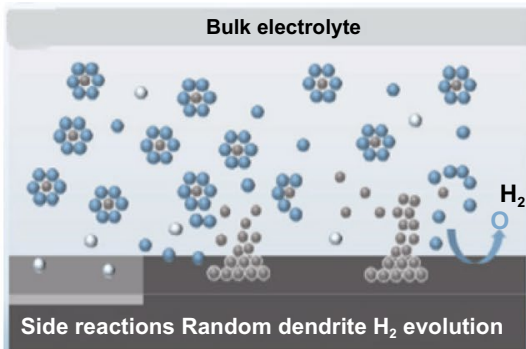

$\mathrm{O}_{2} \odot \mathrm{H}_{2} \mathrm{O}$

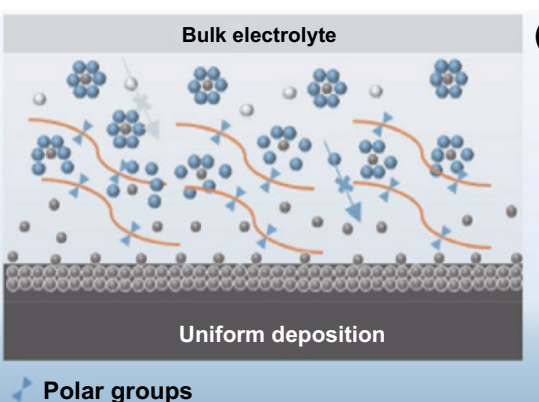

(b)

(d)
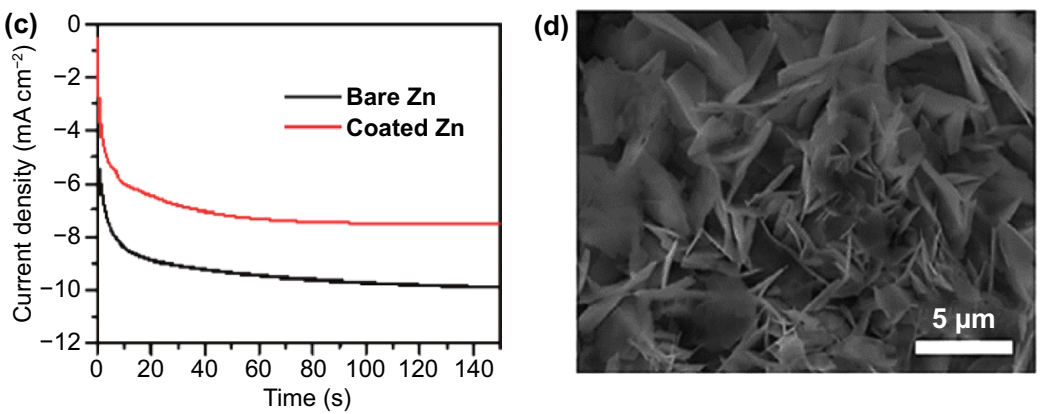

(e)

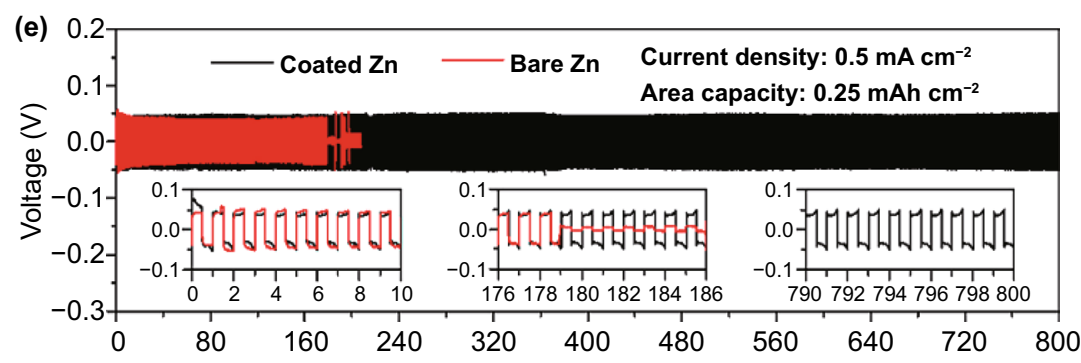

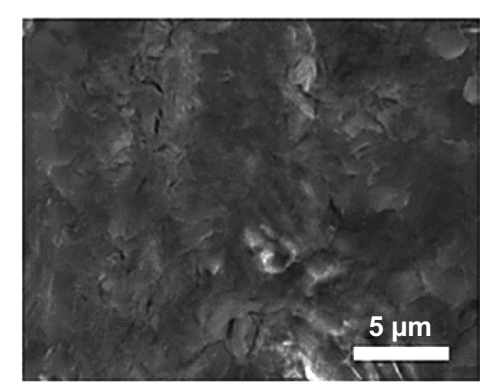

(f) $\mathrm{Zn}^{2+}$ ion flux homogenization

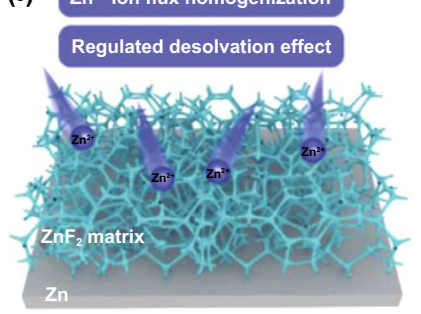

Fig. 4 a Schematic diagram of the mechanism of cyanoacrylate for suppressing $\mathrm{Zn}$ dendrite; $\mathbf{b}$ The illustrations of electronic cloud distribution of cyanoacrylate monomer; $\mathbf{c}$ CAs of bare $\mathrm{Zn}$ and coated $\mathrm{Zn}$ at a $150 \mathrm{mV}$ overpotential; $\mathbf{d}$ Morphology of bare $\mathrm{Zn}$ foil and 502-decorated $\mathrm{Zn}$ foil obtained from symmetric $\mathrm{Zn}$ cells after $\mathrm{Zn}$ stripping/plating for 100 cycles at $0.5 \mathrm{~mA} \mathrm{~cm}{ }^{-2}$ for $0.25 \mathrm{mAh} \mathrm{cm}^{-2}$; e long-term cycling stability for the symmetrical cells at $0.5 \mathrm{~mA} \mathrm{~cm}{ }^{-2}$ for $0.25 \mathrm{mAh} \mathrm{cm}^{-2}$ with the inset showing detailed voltage profile [62]. Copyright 2020, Elsevier. f Schematic illustration of the $\mathrm{Zn} @ \mathrm{ZnF}_{2}$ electrode [67]. Copyright 2020, Wiley-VCH 
alcohol) group in poly(vinyl butyral) (PVB) [41], the carbonyl group in polyimide [49], the amide group and pyrrolidone group in polyacrylamide (PAM)/polyvinylpyrrolidone (PVP) [61], and the cyano group in cyanoacrylate [62] or polyacrylonitrile (PAN) [63]. These polar groups can donate electron pairs, which can guide coordination adsorption through strong interaction with $\mathrm{Zn}^{2+}$ ions (Fig. 4b). Due to the lower energy barrier, $\mathrm{Zn}^{2+}$ ions are transferred along the long organic chain containing polar groups, rather than freely diffused, and a large number of long chains create denser and more uniform nucleation sites for $\mathrm{Zn}$ deposition, which can be sensitively reflected by the variation in current versus time at a constant potential during the deposition process (Fig. 4c) [62]. At the same time, considering the electrostatic repulsion, the electronegative polar groups can block a certain amount of anion ions. It means that $\mathrm{Zn}^{2+}$ ions have a stronger driving force to gather and migrate in the modified layer than other ions or molecules. The priority and selectivity promote the rapid transfer of $\mathrm{Zn}^{2+}$ ions and the decrease in the concentration gradient, which can significantly reduce concentration polarization. As a result, stable $\mathrm{Zn}$ deposition and stripping can be achieved (Fig. 4d, e). Furthermore, polar groups can change the solvated structure of $\left[\mathrm{Zn}\left(\mathrm{H}_{2} \mathrm{O}\right)_{6}\right]^{2+}$ ions to lower desolvation energy. On the one hand, owing to the strong interaction between polar groups and solvated $\mathrm{Zn}^{2+}$ ions, polar groups can replace part or all of the $\mathrm{H}_{2} \mathrm{O}$ molecules; on the other hand, the $\mathrm{N}, \mathrm{O}$, and $\mathrm{F}$ atoms in some polar groups may fix the $\mathrm{H}_{2} \mathrm{O}$ molecules of the solvent sheath by forming hydrogen bonds, resulting in a destroyed sheath structure and reduced coordination number of $\mathrm{Zn}^{2+}$ [64-66]. The enhanced desolvation process can increase the corrosion potential and reduce the corrosion current, indicating less tendency and rate of corrosion and hydrogen evolution. In addition to organic materials, some inorganic materials can also interact strongly with $\mathrm{Zn}^{2+}$ ions, which has a similar effect on concentration field adjustment. For example, a three-dimensional (3D) interconnected $\mathrm{ZnF}_{2}$ matrix could be obtained by electrodeposition in $\mathrm{NH}_{4} \mathrm{~F}$ aqueous solution [67]. Compared to bare $\mathrm{Zn}$ mental, the $\mathrm{ZnF}_{2}$ matrix exhibits stronger Coulomb attraction for $\mathrm{Zn}^{2+}$ ions. Coupled with the specific interconnected porous structure, the $\mathrm{Zn}^{2+}$ ion flux becomes more uniform, and the desolvation effect and transfer kinetics are enhanced (Fig. 4f). In addition, the $\mathrm{S}, \mathrm{O}$, and $\mathrm{P}$ atoms in $\mathrm{ZnS}, \mathrm{ZnO}$, and $\mathrm{ZnP}$ layers, respectively, also have strong adsorption to $\mathrm{Zn}^{2+}$ ions, which have been proven to contribute to the concentration field redistribution effect [68-70].

The dielectric material can respond to the external electric field in an inductive manner, producing an electric dipole moment or a change in the electric dipole moment along the direction of the electric field [71]. The directional polarization electric field of the electric dipole moment can adjust the flux of ion charge migration. Precisely, under the applied electric field, the charge in some dielectric materials can move in the microscopic range, resulting in polarization [72]. The additional electric field caused by the polarized charge can manipulate the $\mathrm{Zn}^{2+}$ ion migration. It is recently reported that perovskite-type dielectric material $\mathrm{BaTiO}_{3}$ (BTO) can be polarized by an external field [73], and the Ti ions in $\left[\mathrm{TiO}_{6}\right]^{2+}$ deviate from the center of the symmetrical position to form an aligned electric dipole (Fig. 5a). Whether in the charging or discharging phase, the excited directional polarization electric field can induce the ordered $\mathrm{Zn}^{2+}$ ion migration (Fig. 5b). Impressively, during the plating process, the direction of the polarization electric field can be switched according to the reversal of the external electric field, which simultaneously accelerates the transfer of $\mathrm{Zn}^{2+}$ ions, repels anions, and enhances desolvation (Fig. 5c). As a result, the BTO@Zn symmetric cell exhibits decent cycling stability over $1500 \mathrm{~h}$ at $5 \mathrm{~mA} \mathrm{~cm}{ }^{-2}$ with the capacity of 2.5 $\mathrm{mAh} \mathrm{cm}{ }^{-2}$ (Fig. 5d). Additionally, the previously reported $\mathrm{ZrO}_{2}$-modified layer can form Maxwell-Wagner polarization due to its high dielectric constant and low conductivity compared to $\mathrm{Zn}$ metal [74]. The polarization electric field provides controllable nucleation sites for $\mathrm{Zn}^{2+}$ ions and promotes fast ion kinetics, leading to uniform $\mathrm{Zn}$ deposition/ stripping. Encouragingly, the types of dielectric materials are relatively abundant, which can provide more selectivity for the future design of directional polarization electric fields to redistribute concentration field.

\subsubsection{Redistribution of Electric Field}

In addition to directly changing the flux of molecules and ions on the electrolyte side at the electrolyte/anode interface, directly regulating the electron distribution on the anode side can also manipulate the $\mathrm{Zn}$ deposition behavior. The redistribution of electrons can change the distribution of the electric field, mainly homogenizing the local electric field or enlarging the local electric field. 
(a)

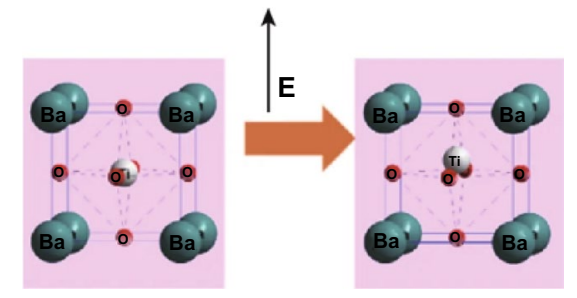

(b)

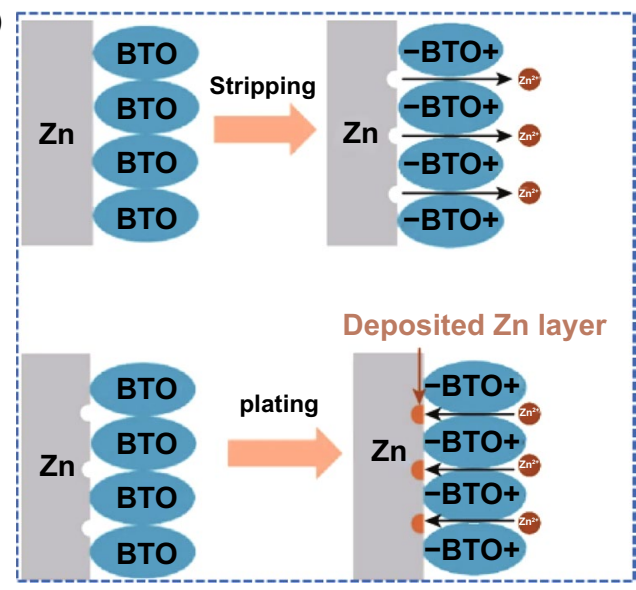

(c)
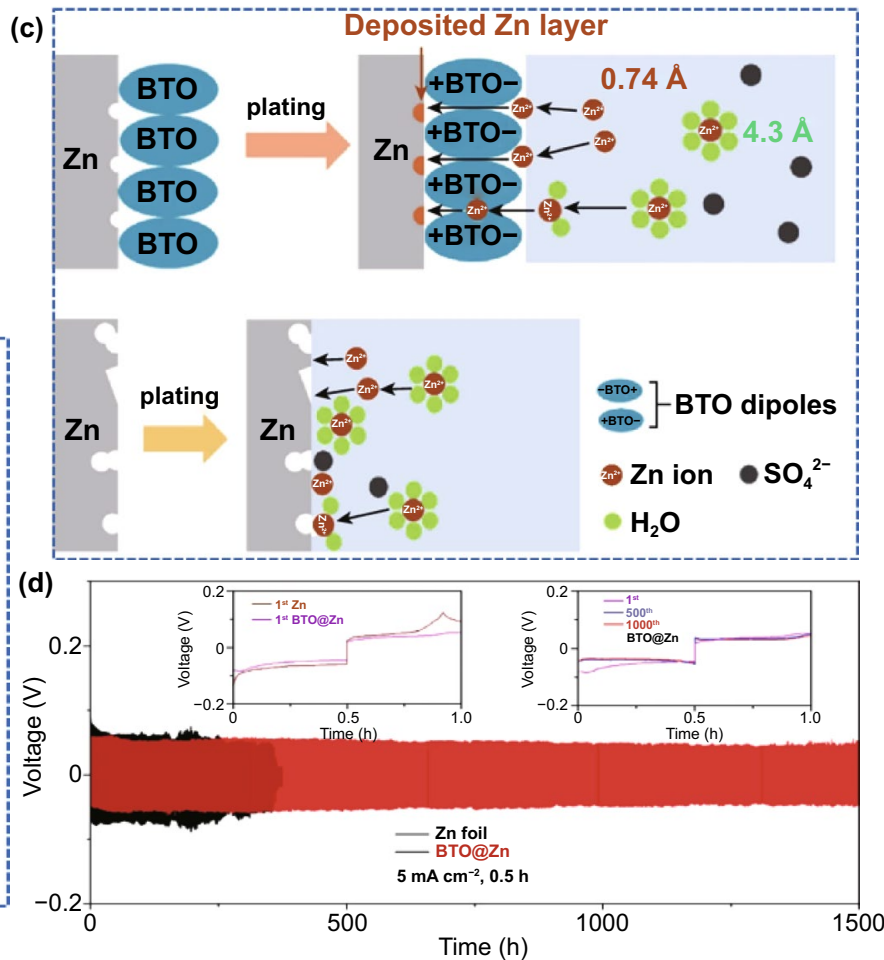

Fig. 5 a Schematic diagram of the Ti ion migration in the $\left[\mathrm{TiO}_{6}\right]$ octahedral interstitial sites under the external electric field; $\mathbf{b}$ Schematic of $\mathrm{Zn}^{2+}$ ion transport during Zn stripping/plating for BTO@Zn foil; c Schematic of the mechanism of $\mathrm{Zn}^{2+}$ ion transport at the (top) BTO@Zn/ electrolyte and (bottom) $\mathrm{Zn}$ anode/electrolyte interface during $\mathrm{Zn}$ plating process; $\mathbf{d}$ Cycling performance of the symmetric cells with $\mathrm{Zn}$ and BTO@ $\mathrm{Zn}$ at $5 \mathrm{~mA} \mathrm{~cm}^{-2}$ with a capacity of $2.5 \mathrm{mAh} \mathrm{cm}^{-2}$. The insets reveal the detailed corresponding voltage profiles at various current densities and different cycles [73]

The deposition behavior of $\mathrm{Zn}^{2+}$ ions dominated by the electric field is greatly affected by the 2D diffusion around the nucleation site. According to the fundamentals of the tip effect, the uneven electric field distribution is caused by the larger local surface charge density. Therefore, the local electric field strength is closely related to the local current density. It can be known from Eq. 3 that while increasing the anode current density in pursuit of a faster battery charging rate, the local current density at the $\mathrm{Zn}$ deposition site should be reduced to inhibit the growth of dendrites [30]. Under the premise of a constant applied current, the modified conductive layer with a large specific surface area, such as graphene oxide (GO) [75], reduced graphene oxide (rGO) [76, 77], graphite [78], carbon nanotubes (CNT) [79], and other carbon-based materials, can distribute a part of the electronic charge of the anode, thereby having high electrochemical activity. This provides more selective nucleation sites for $\mathrm{Zn}$ deposition instead of converging only in the initial few hot spots for charge transfer. Thus, the local current density is significantly reduced, corresponding to a more uniform local electric field [80]. A novel and simple strategy for constructing a graphite functional interface was proposed as a proof of concept. The graphite layer was painted directly on the anode surface with the assistance of ordinary pencils (Fig. 6a) [78]. Through electrochemical tests, the nucleation overpotential (NOP) value $(69 \mathrm{mV})$ of pure $\mathrm{Zn}$ anode was much higher than that of graphite-coated $\mathrm{Zn}$ anode $(9 \mathrm{mV})$ (Fig. 6b). This meant that the high conductivity and large surface area of the graphite layer enabled the anode with a lower local current density, which corresponds to the characteristics of smaller $\mathrm{Zn}$ nuclei formation and no dendrites. This result was also proved by observing the transparent symmetrical battery through an optical microscope: Some large dendrites (dark spots marked by yellow arrows) were formed on the bare $\mathrm{Zn}$ surface within 40 min plating, while the graphite-coated $\mathrm{Zn}$ anode had a flat and smooth surface (Fig. 6c). Apart from carbon-based materials, 2D-structured MXene material with metallic conductivity as a modified layer also has a similar effect of homogenizing the local 

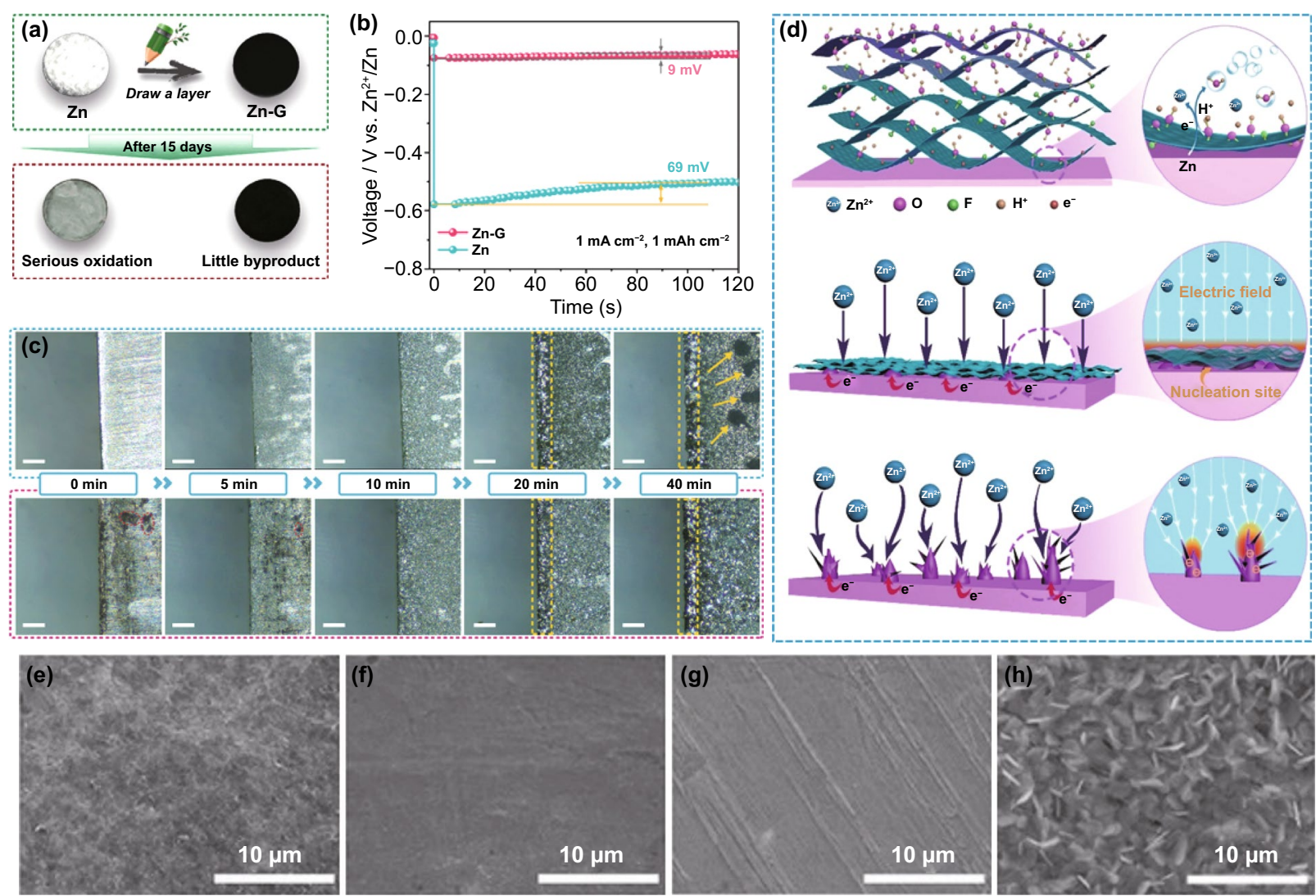

Fig. 6 a Schematic illustration of the modification process and the stability in $2 \mathrm{M} \mathrm{ZnSO}_{4}$ electrolyte of $\mathrm{Zn}$ and graphite-coated $\mathrm{Zn}$ anode; b The voltage-time curves of $\mathrm{Zn}$ and $\mathrm{Zn}-\mathrm{G}$ symmetric cells at $1.5 \mathrm{~mA} \mathrm{~cm}{ }^{-2}$; $\mathbf{c}$ In situ optical microscope photographs of (top) $\mathrm{Zn}$ and (bottom) $\mathrm{Zn}-\mathrm{G}$ electrodes observed by symmetric transparent cells under various deposition times [78]. Copyright 2020, Wiley-VCH. d Illustration of (top) synchronously reducing and assembling MXene layer on the $\mathrm{Zn}$ foil surface; Illustration of $\mathrm{Zn}$ plating behavior of (middle) MXene-coated $\mathrm{Zn}$, and (bottom) pure Zn; SEM images of MZn-60 and pure Zn e, g before cycling, and $\mathbf{f}, \mathbf{h}$ after 100 cycles at $3 \mathrm{~mA} \mathrm{~cm}{ }^{-2}$ [81]. Copyright 2020, Wiley-VCH

electric field (Fig. 6d) [81]. Even at the large current density, the highly conductive structure network can destroy the tip effect inside the MXene $\left(\mathrm{Ti}_{3} \mathrm{C}_{2} \mathrm{~T}_{\mathrm{x}}\right)$ layer by eliminating the uneven local electric field. Due to the lack of driving force for dendrite growth, the nucleation and growth of $\mathrm{Zn}$ are more uniform (Fig. 6e-h).

Due to the anode electron redistribution, the possible deposition sites of $\mathrm{Zn}^{2+}$ ions include the $\mathrm{Zn}$ metal surface, inside, and the surface of the modified conductive layer. The electrical conductivity of some modified layers is lower than that of $\mathrm{Zn}$ metal, and there is also generally contact resistance between them, such as ZIF-8 derived carbon [82]. Different electrical conductivity and high contact resistance result in potential change between the modified layer and $\mathrm{Zn}$ metal. The potential near the $\mathrm{Zn}$ metal surface was low (or negative) enough for $\mathrm{Zn}^{2+}$ reduction. Therefore, $\mathrm{Zn}$ deposition will preferentially occur at a low potential on the more conductive $\mathrm{Zn}$ metal surface, leading to a positionselected, bottom-up $\mathrm{Zn}$ deposition process [44]. However, the excellent conductive network has fast electron transfer capability for anodes modified with most carbon-based materials or MXene materials. There is almost no potential change between the modified layer and $\mathrm{Zn}$ metal. At a reasonable potential, $\mathrm{Zn}$ reduction will occur as long as it contacts the conductive network. Thus, the excellent conductive modified layer is the preferred location for $\mathrm{Zn}$ deposition [28]. For example, rGO with a layered structure can generate a stable electric field in which the electrolyte is fully penetrated during the plating process. The $\mathrm{Zn}^{2+}$ ions are preferentially distributed uniformly rather than aggregated; $\mathrm{Zn}$ is deposited on the inside and surface of the $\mathrm{rGO}$ layer (Fig. 7a-c) [76, 77]. It is worth noting that most of 
the conductive network materials are not structurally complete but have edges and defects on which $\mathrm{Zn}^{2+}$ ions will be deposited preferentially. At the same time, the $\mathrm{Zn}^{2+}$ ions transferred from the electrolyte will first contact the surface of the modified layer, suggesting that $\mathrm{Zn}$ deposited on the surface has a higher priority than the interior. Still, the modified layer surface cannot play the role of homogenizing the electric field. These all mean that the modified layer with a conductive network can only uniform the electric field and suppress dendrites within a certain range. Moreover, due to the increased area contact with the water in the electrolyte, the problem of side reactions at the reaction interface is still troublesome.

Contrary to the strategy of homogenizing the electric field to suppress the $2 \mathrm{D}$ diffusion of $\mathrm{Zn}^{2+}$ ions, constructing protrusions on the metal surface to enlarge the local electric field to strengthen the 2D diffusion can also suppress dendrites, but the premise is that the protrusions are uniformly and densely distributed on the anode surface [83]. For example, a large number of Au nanoparticles (AU-NP) are constructed on the surface of $\mathrm{Zn}$ metal by magnetron sputtering (Fig. 7d, e) [84]. Seed crystals have high curvature and large local electric fields around them, which will preferentially become sites for $\mathrm{Zn}$ deposition and growth. The uniform and dense seed crystals enable the deposition of $\mathrm{Zn}^{2+}$ ions to be universal instead of growing on several loose tips on the bare Zn surface (Fig. 7f). Actually, during the $\mathrm{Zn}$ deposition process, the previously generated $\mathrm{Zn}$ nuclei also form a large local electric field to affect subsequent nucleation and growth. Therefore, adjusting the initial nucleation site is of great significance for realizing a dendrite-free anode.

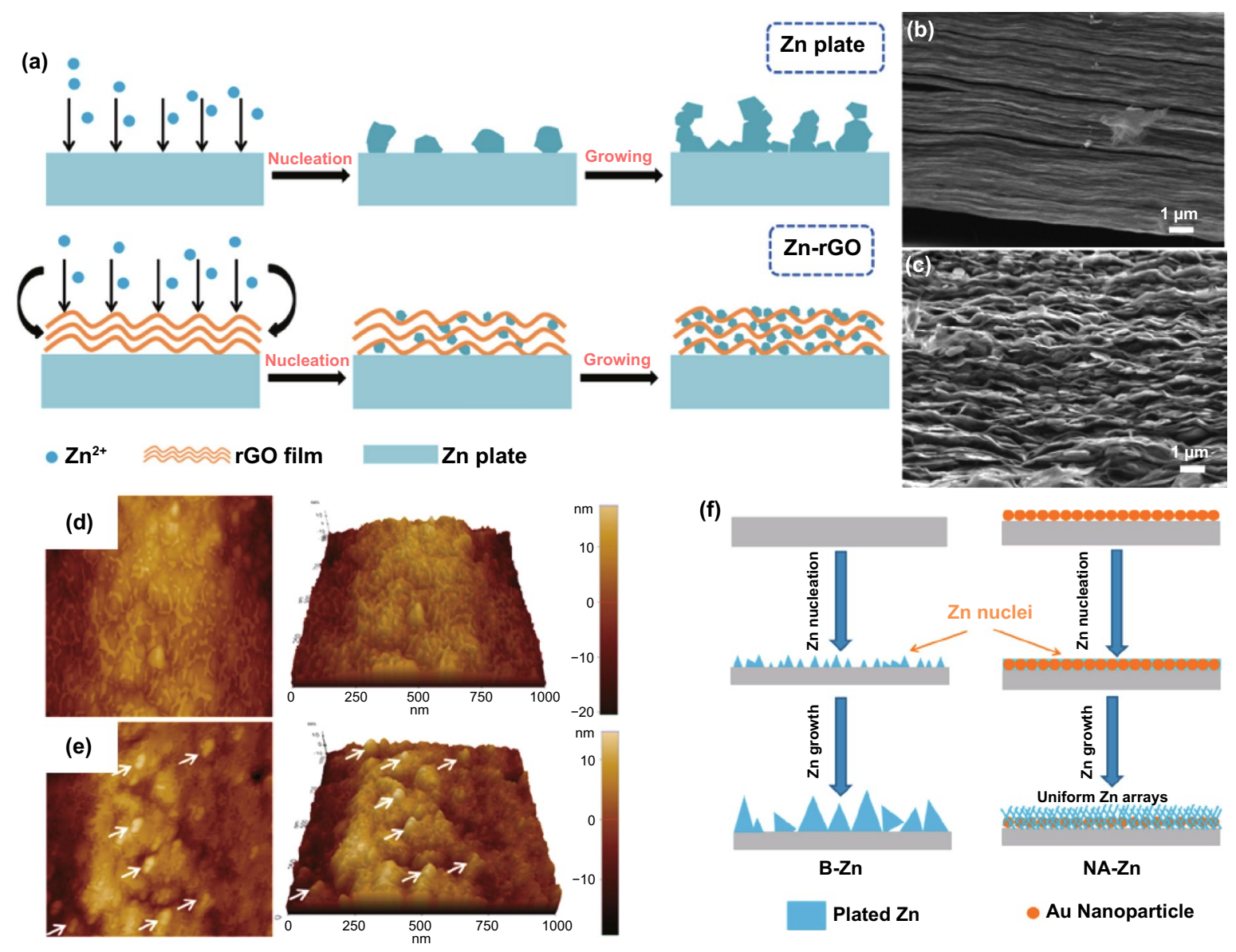

Fig. 7 a Schematic illustrating the Zn plating behavior of the bare $\mathrm{Zn}$ and $\mathrm{Zn} / \mathrm{rGO}$ anodes; cross-sectional SEM images of rGO film on Zn foil b before cycling and $\mathbf{c}$ after cycling [77]. Copyright 2019, Elsevier. Atomic force microscope images of $\mathbf{d}$ bare $\mathrm{Zn}$ anode and e Au-decorated $\mathrm{Zn}$ anode; $\mathbf{f}$ Schematic illustration of the Zn stripping/plating process [84]. Copyright 2019, American Chemical Society 


\subsubsection{Regulation of Surface Binding Energy}

According to thermodynamics, $\mathrm{Zn}$ nucleation is controlled by the decrease in free energy due to phase transition and the increase in surface energy due to new interfaces. The former is the driving force for nucleation, and the latter leads to a nucleation barrier. Zn preferentially nucleates at the thermodynamically favorable adsorption sites of low energy barriers [85]. The modified material with high $\mathrm{Zn}$ affinity has a lower nucleation energy barrier, thus leading to a smaller nucleation overpotential, which is more conducive to $\mathrm{Zn}$ nucleation. The $\mathrm{Zn}$ affinity is evaluated by comparing the binding energy (calculated by density functional theory) of the $\mathrm{Zn}$ atoms attached to the modified layer and the $\mathrm{Zn}$ metal surface, and the larger one (more negative) has a higher $\mathrm{Zn}$ affinity [86]. It is reported that some metals have high $\mathrm{Zn}$ affinity, such as $\mathrm{Sn}, \mathrm{In}, \mathrm{Ag}$, and $\mathrm{Cu}$; they can serve as heterogeneous seeds to induce $\mathrm{Zn}$ nucleation and growth [87-90]. Compared with Zn metal or carbon, a wellorganized Sn layer has a uniform and densely distributed zincophilic sites on the surface [87]. During the Zn nucleation process, $\mathrm{Zn}$ has stronger electronic interactions with these zincophilic sites (Fig. 8a), and thus, Zn preferentially nucleates uniformly at these sites. The uniformly distributed $\mathrm{Zn}$ nuclei also guide the subsequent uniform $\mathrm{Zn}$ deposition (Fig. 8b). Therefore, the zincophilic layer can significantly inhibit dendrite growth (Fig. 8c, d). Besides, it was previously proposed that the Li affinity of carbon materials can be enhanced by heteroatom doping, which can be explained by electronegativity, local dipole, and charge transfer. Similarly, the introduction of $\mathrm{N}$-containing sites significantly changes the $\mathrm{Zn}$ affinity of graphene [50]. Widely distributed pyridine sites induce the spacious distribution of the initial $\mathrm{Zn}$ nuclei by forming $\mathrm{Zn}-\mathrm{N}$ bonds. Lateral $\mathrm{Zn}$ growth leads to nearby connections to form $\mathrm{Zn}$ clusters, resulting in uniform $\mathrm{Zn}$ deposition (Fig. 8e-g).

Since atoms, molecules, or ions belonging to the noncubic crystal system have multiple crystal facets, different crystal facets have different binding energies to $\mathrm{Zn}$. Thus, even in the modification layer of the same material, different exposed crystal facets have other effects on $\mathrm{Zn}$ nucleation. Consider as-prepared faceted $\mathrm{TiO}_{2}\left(\mathrm{~F}-\mathrm{TiO}_{2}\right)$ nanosheets with exposed $\left(\begin{array}{lll}0 & 0 & 1\end{array}\right)$ and $\left(\begin{array}{lll}1 & 0 & 1\end{array}\right)$ facets and commercial $\mathrm{TiO}_{2}\left(\mathrm{C}-\mathrm{TiO}_{2}\right)$ with exposed $\left(\begin{array}{lll}1 & 0 & 0\end{array}\right)$ facets, the former with a lower $\mathrm{Zn}$ affinity can repel the $\mathrm{Zn}$ adsorption, ensuring that
$\mathrm{Zn}^{2+}$ ions preferentially accumulate and nucleate on the $\mathrm{Zn}$ metal under the $\mathrm{F}_{-} \mathrm{TiO}_{2}$ nanosheets; on the contrary, the high $\mathrm{Zn}$ affinity of the latter leads to preferential $\mathrm{Zn}$ deposition on the $\mathrm{C}-\mathrm{TiO}_{2}$ nanosheet surface (Fig. 9a, b) [86]. It is worth noting that the affinity reflects the difficulty of $\mathrm{Zn}$ deposition on the substrate. Providing a lower nucleation energy barrier promotes the kinetics of $\mathrm{Zn}$ deposition but does not necessarily change the crystal plane orientation of $\mathrm{Zn}$ deposition and form a $\mathrm{Zn}$ surface texture [91]. As the area capacity increases, it may be beyond the controllable ability of zincophilic sites to $\mathrm{Zn}$ deposit; $\mathrm{Zn}$ dendrites will still form. The restricted deposition space under the modified layer can further inhibit the large-scale dendrite growth. In contrast, the surface of the modified layer no longer has a constraining effect, and the battery is more likely to be damaged by dendrites (Fig. 9c) [86]. As a similar proof, it has recently been reported that the Sn (llll) surface has a higher $\mathrm{Zn}$ affinity than the Sn $\left(\begin{array}{lll}2 & 0 & 0\end{array}\right)$ surface, and both of them are higher than commercial $\mathrm{Zn}$ foils (Fig. 9d). However, the $\mathrm{Zn}$ anode with the Sn (llll 011$)$ surface texture layer still exhibits rough $\mathrm{Zn}$ deposition morphology, while the deposition morphology on the Sn $\left(\begin{array}{lll}2 & 0 & 0\end{array}\right)$ surface texture layer is uniform and smooth. This is derived from the latter's high average surface energy which may result in particularly strong capillary action, leading to better deposit "wettability" (Fig. 9e, f) [91]. According to the above analysis, the binding energy significantly influences the initial $\mathrm{Zn}$ nucleation behavior. At the large area capacity, the modified layer that dominates the $\mathrm{Zn}$ deposition with high binding energy does not necessarily play a key role in alleviating the dendrite problem or even may cause negative effects. It is worth mentioning that in the hexagonal close-packed (hcp) $\mathrm{Zn}$ lattice, the $\mathrm{Zn}$ (l $\left.\begin{array}{lll}0 & 2\end{array}\right)$ crystal surface has a smooth surface and uniform interface charge density, which is not conducive to the formation of dendrites. And such a plane with high atomic coordination has high binding energy, ensuring preferential $\mathrm{Zn}$ deposition at these more advantageous sites (Fig. 9g-i). During the deposition process, the surface texture of the $\mathrm{Zn}$ $\left(\begin{array}{lll}0 & 0 & 2\end{array}\right)$ planes can lead to a spontaneous reorientation of $\mathrm{Zn}$ crystallites and, finally, maintain the horizontal growth of the Zn (lll $\left.0 \begin{array}{ll}0 & 2\end{array}\right)$ planes through the epitaxial mechanism. At the same time, due to the low electrochemical activity of $\mathrm{Zn}\left(\begin{array}{lll}0 & 0\end{array}\right)$ planes, corrosion and $\mathrm{H}_{2}$ evolution may also be reduced [92, 93]. Therefore, adjusting the surface binding energy by constructing the modified layers and controlling 

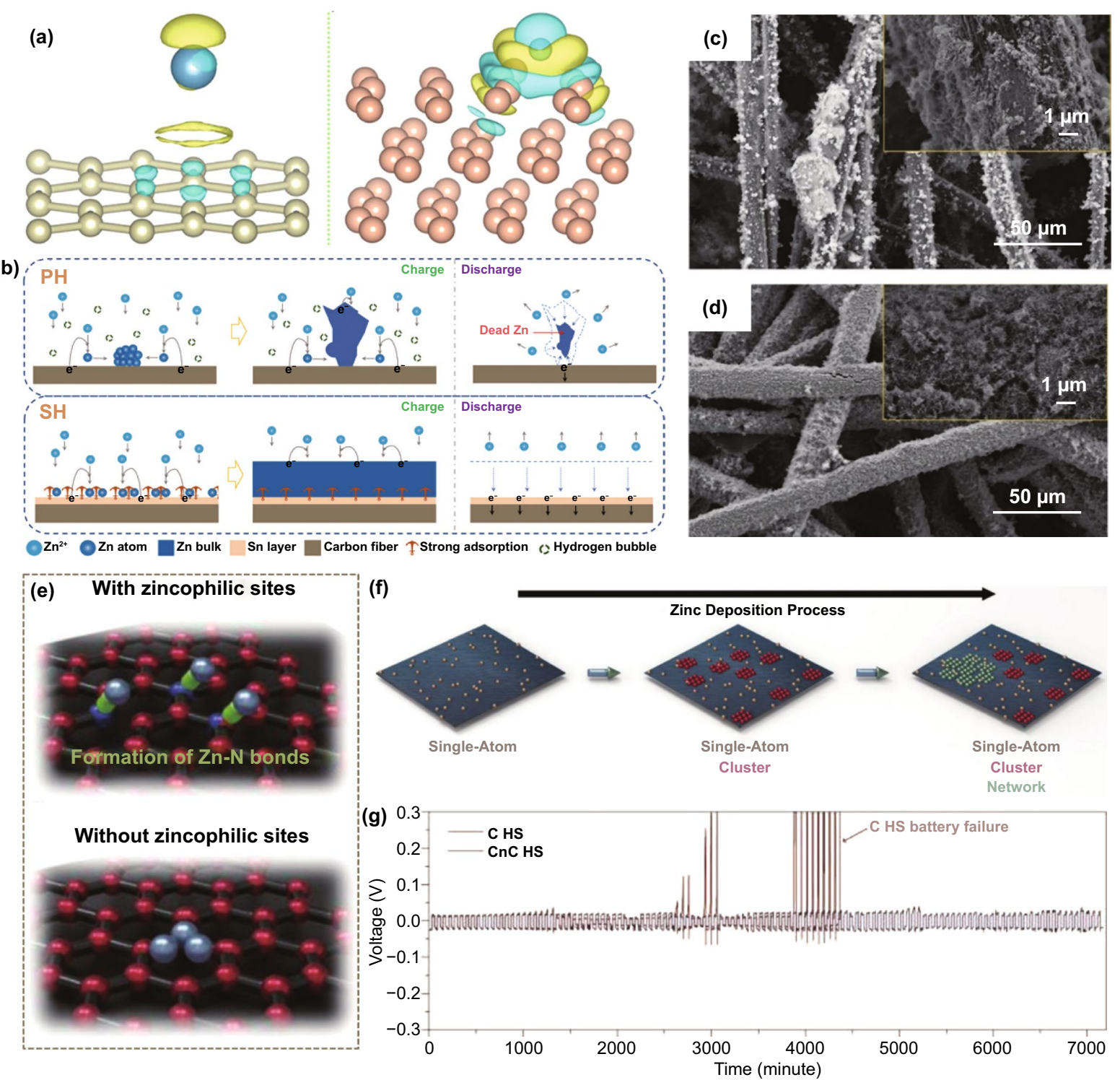

Fig. 8 a Interfacial charge density of (right) carbon and (left) Sn; b Schematic illustration of Zn deposition induction mechanism; SEM images of $\mathbf{c} \mathrm{PH}$ and $\mathbf{d}$ SH after charging (inset, higher magnification) [87]. Copyright 2019, Wiley-VCH. e (top) Spacious Zn nucleation with zincophilic sites and (bottom) dense nucleation on zincophobic surface; f Schematic illustration of Zn deposition on surface with zincophilic sites; $\mathbf{g}$ Galvanostatic cycling performance of symmetric cells with and without zincophilic nitrogen sites [50]. Copyright 2020, Wiley-VCH

their exposed crystal planes is beneficial for obtaining a stable anode interface and deeply understanding the internal mechanism of binding energy on $\mathrm{Zn}$ deposition.

\subsection{Structural Anode}

Numerous reports have confirmed that the structural design can effectively enhance the overall performance of the ZIBs. Diversified structural design is more and more favored by subsequent research work. The modified layer strategy is mainly based on the $\mathrm{Zn}$ deposition of the metal anode plate, which has a limited contact area with the electrolyte. Differently, extending the contact surface to 3D space can significantly improve anode designability. Drastically increased specific surface area is the most crucial feature of structured anodes. Although this may enhance the formation of side reactions, it can effectively increase $\mathrm{Zn}$ nucleation sites and reduce local current density. Moreover, sufficient contact 


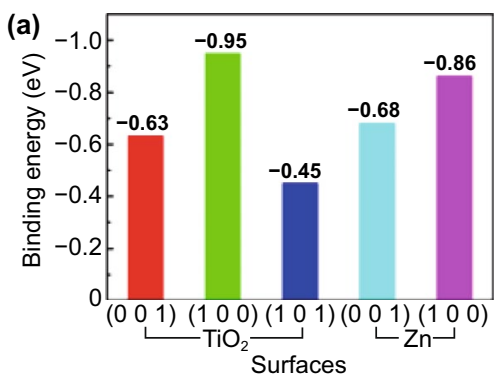

(b)

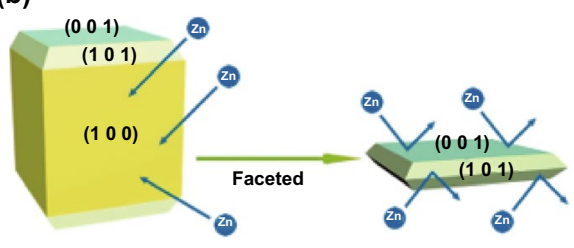

$\mathrm{TiO}_{2}$ bulk $\quad \mathrm{TiO}_{2}$ sheet

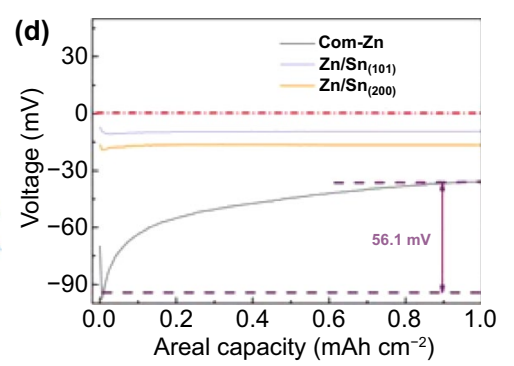

(e)

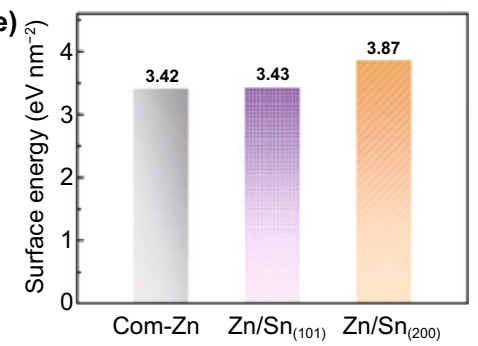

Com-Zn Zn/Sn $\mathrm{Z}_{(101)} \mathrm{Zn} / \mathrm{Sn}_{(200)}$

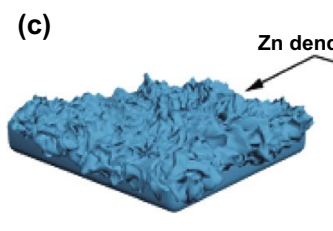

ZF

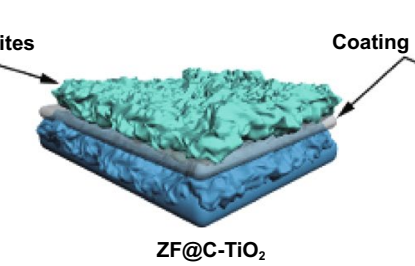

$\mathrm{ZF} @ \mathrm{C}-\mathrm{TiO}_{2}$
Coating layers

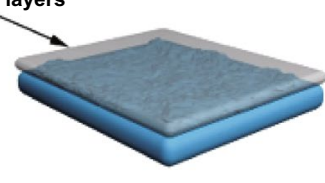

ZF@F-TiO

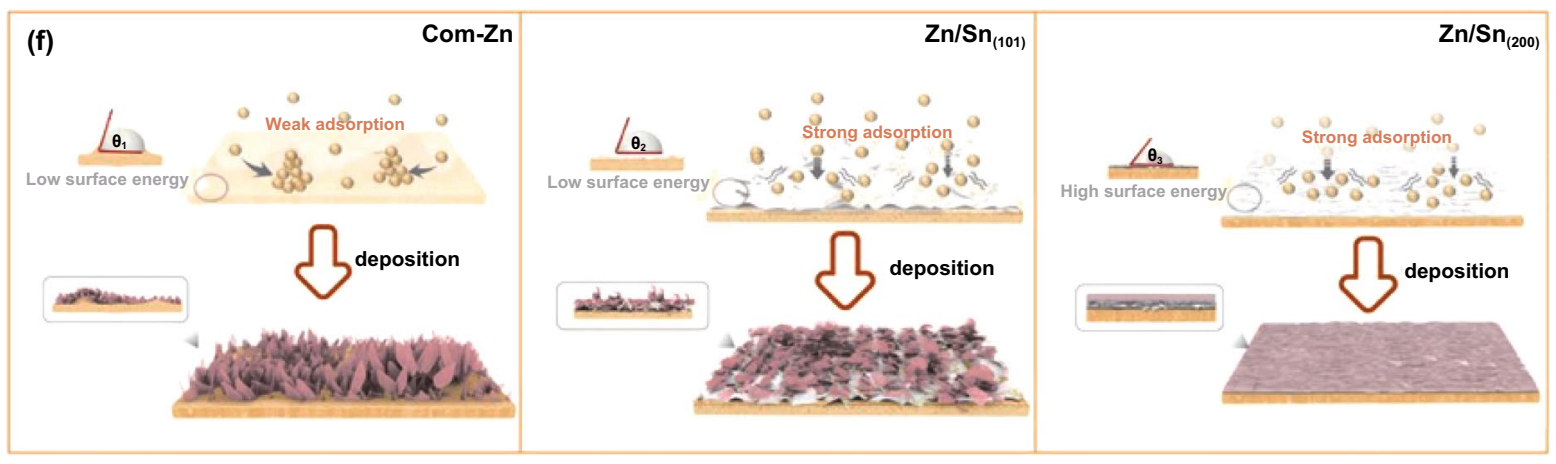

(g)

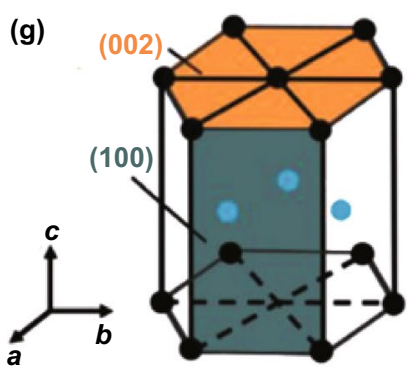

(h)

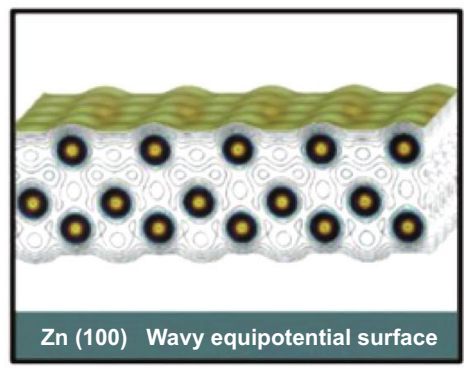

(i)

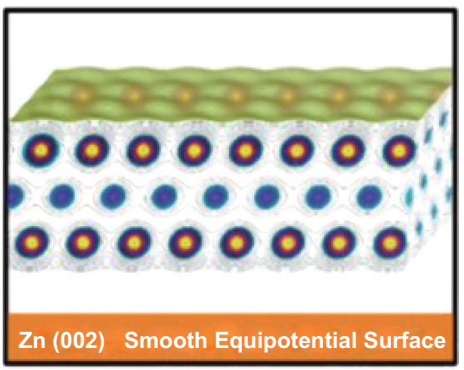

Fig. 9 a Calculated binding energies of $\mathrm{Zn}$ atom with different facets; $\mathbf{b}$ Schematic illustration of the interaction between $\mathrm{Zn}$ and anatase $\mathrm{TiO}{ }_{2}$ with different exposed facets; $\mathbf{c}$ Schematic illustration of the $\mathrm{Zn}$ plating process with different coating layers [86]. d Voltage profiles of $\mathrm{Zn}$ deposition on Com-Zn, Zn/Sn (101), and Zn/Sn (200) at a current density of $1 \mathrm{~mA} \mathrm{~cm}{ }^{-2}$ and a capacity of $1 \mathrm{mAh}^{-2}$; e The calculated surface energy on different electrodes; f Schematic illustration of Zn deposition process on Com-Zn, Zn/Sn (101) and Zn/Sn (200) [91]. Copyright 2020, Wiley-VCH. $\mathbf{g}$ The structure of metal Zn; Surface atomic arrangement and electron equipotential plane of $\mathbf{h} \mathrm{Zn}(100)$ and $\mathbf{i} \mathrm{Zn}(002)$ [92]. Copyright 2021, Wiley-VCH

with the electrolyte and rapid charge transfer allows for lower polarization. These are conducive to obtaining a stable anode without dendrites. The reported structured anode materials, which can be roughly classified into the metallic matrix material, carbonaceous matrix material, and other matrix materials, exhibit great potential for regulating the Zn deposition behavior.
To obtain long-lifespan reversible cycle performance, the structured anode that plays a physical structural support role should have good structural stability, which puts forward high requirements for suppressing dendrites. Metal-based structured anodes, especially $\mathrm{Cu}$-based materials (Cu mesh, $\mathrm{Cu}$ foam [94, 95], and porous $\mathrm{Cu}$ [96]) with excellent $\mathrm{Zn}$ affinity, can maintain the morphology of the structure due 
to their rigid properties. Zn nucleation and growth can be restricted to a specific structure through deliberate design, thereby inhibiting dendrites. Based on the unique structure and function of the $\mathrm{Cu}$ mesh, modifying the $\mathrm{Cu}$ mesh with $\mathrm{CuO}$ nanowires can further expand the specific surface area of the current collector and simultaneously adjust the ion distribution and electric field at the anode (Fig. 10a, b) [97]. Due to its lower nucleation energy barrier, $\mathrm{CuO}$ nanowires tend to selectively absorb $\mathrm{Zn}^{2+}$ ions and can be reduced to $\mathrm{Cu}$ nanowires to form a staggered 3D copper matrix. $\mathrm{Zn}$ can be uniformly deposited in the gaps of the nanowires without dendrite formation (Fig. 10c). Similarly, a 3D Ni-Zn anode with a multi-channel lattice structure was fabricated with the help of 3D printing technology (Fig. 10d) [98]. Compared with planar electrodes, 3D Ni-Zn has a larger specific surface area, which redistributes the local electric field and induces the preferential and uniform $\mathrm{Zn}$ deposition into the 3D microchannels, thus successfully suppressing dendrites and significantly improving the electrochemical performance of the battery. Besides, $\mathrm{Zn}$ itself is also designed as the main body of the 3D structure [99-101]. For example, a 3D porous $\mathrm{Zn}$ anode with dual channels, consisting of a continuous cavity and a conductive framework, allows ions and electrons to migrate quickly at the anode interface [99]. The unique cavity structure limits the Zn deposition position, thereby inhibiting dendrite growth (Fig. 10e). Recently, it has been proposed that nanoporous $\mathrm{Zn}$ electrodes ( $\mathrm{npZn}$ ) with controllable pore size can be prepared by the alloying-dealloying method. When the pore size is small enough $(<40 \mathrm{~nm})$, the space charge will significantly affect the effective ion concentration of the electrolyte in the pore. Cations $\left(\mathrm{Zn}^{2+}\right)$ are enriched, and anions are reduced so that interface-localized concentrated electrolytes can be achieved (Fig. 10f-h). Zn anode with a nanoporous structure can promote the uniform $\mathrm{Zn}$ plating and suppress side reactions [100].

Besides, the carbonaceous matrix materials have an open 3D skeleton structure; their mechanical flexibility can sufficiently cope with the change in anode volume during cycling, particularly adaptable to shapeable anodes applied to flexible and wearable electronic devices, such as fiber-shaped Zn-ion micro-batteries [102, 103]. Up to now, several excellent research works have developed multiple carbon-based structure anodes, mainly based on highly conductive carbon fiber matrix, including carbon cloth [104], graphite felt [105], carbon nanotubes [106], etc. Impressively, the application of flexible 3D carbon nanotubes (CNT) scaffold on $\mathrm{Zn}$ anode has attracted much attention (Fig. 11a) [106]. The interconnected CNT formed on the carbon fiber cloth can successfully lower the energy barrier of Zn nucleation, and the uniform electric field distribution ensures the reversible plating/ stripping of the dendrite-free anode (Fig. 11b). Generally, $\mathrm{Zn}$ on a carbon-based host is formed in situ in advance by many methods, such as electrodeposition or vapor deposition. Recently, by using a vacuum filtration process, active $\mathrm{Zn}$ powder can also be compounded on the carbon network to form a 3D porous $\mathrm{Zn}$ anode (Fig. 11c) [107]. It can be understood that point-to-point contact between $\mathrm{Zn}$ powder particles may form a vulnerable conductive network. During the $\mathrm{Zn}$ stripping process, owing to the short electron pathway, the contact points are likely to be dissolved first, and the $\mathrm{Zn}$ powder particles will easily lose contact with the electrode matrix and cause Zn death [108]. However, the carbon fiber skeleton can act as a binder to firmly bond the $\mathrm{Zn}$ powder, which can effectively reduce the capacity loss caused by Zn dissolution (Fig. 11d). Although not as large as the specific surface area of nanoscale $\mathrm{Zn}$ by in situ nucleation and growth, a large number of micron-scale pores between powder particles or carbon fiber skeletons in the electrode provide enough space for $\mathrm{Zn}$ deposition and dendrite growth, resulting in stable anode performance (Fig. 11e). The adjustable surface area of $\mathrm{Zn}$ powder has practical significance for commercial production. It is worth beware that the $\mathrm{Zn}$ charge transfer resistance decreases with the particle size of the $\mathrm{Zn}$ powder, implying that the smaller particle size $\mathrm{Zn}$ powder has a higher reactivity. Still, at the same time, it is accompanied by enhanced hydrogen evolution and corrosion reaction (Fig. 11f). Thus, the $\mathrm{Zn}$ powder particle size in the carbon fiber framework should be designed reasonably. Additionally, it has been reported that a similar atomic arrangement between graphene and $\mathrm{Zn}$ metal results in low lattice mismatch [109]. During the deposition process, $\mathrm{Zn}^{2+}$ ions will heteroepitaxially nucleate along the graphene crystal plane and grow in a strain-free state, and then $\mathrm{Zn}^{2+}$ ions continue to deposit homogeneously epitaxially on the newly formed $\mathrm{Zn}$ metal layer (Fig. 11g). This Zn deposition pattern that locks the crystal orientation relationship can fundamentally eliminate the growth of dendrites. The reversibility of the epitaxial $\mathrm{Zn}$ anode guided by the graphene substrate is greatly improved, and the CE still exceeds $99 \%$ even after 1000 cycles. Although there is a lack of strong evidence to prove that the $\mathrm{Zn}$ deposits on graphene sheets are oriented, this 
(a)

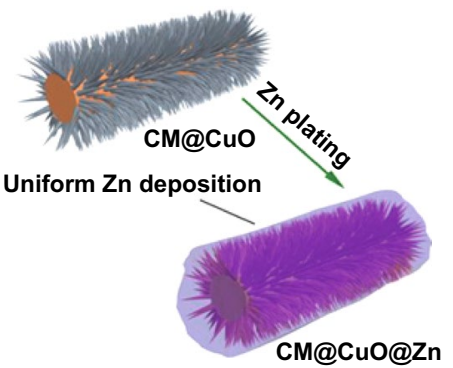

(c)

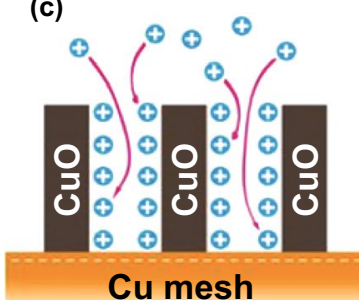

\section{Nucleation}

(b)
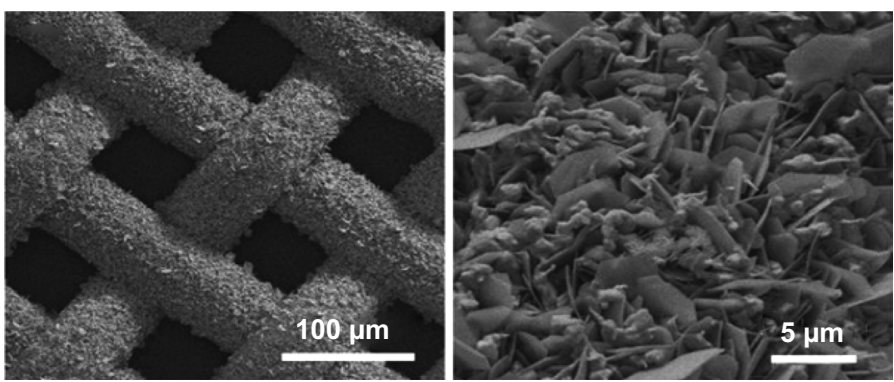

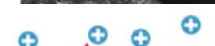

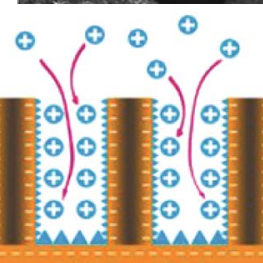

Cu mesh

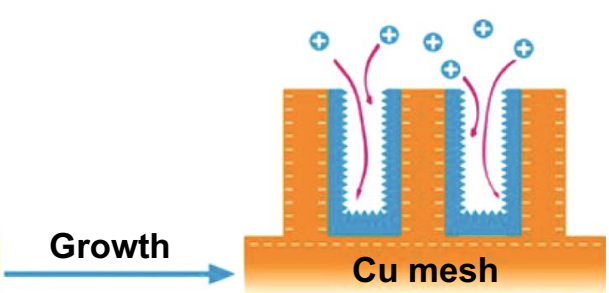

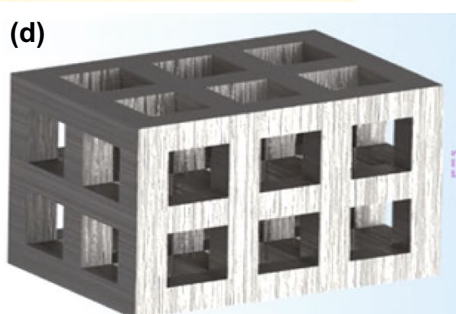

3D Ni

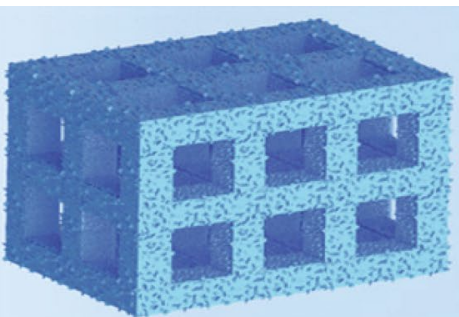

Initial Zn-plating

(e)

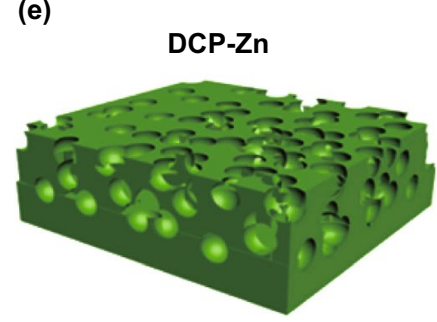

(g)

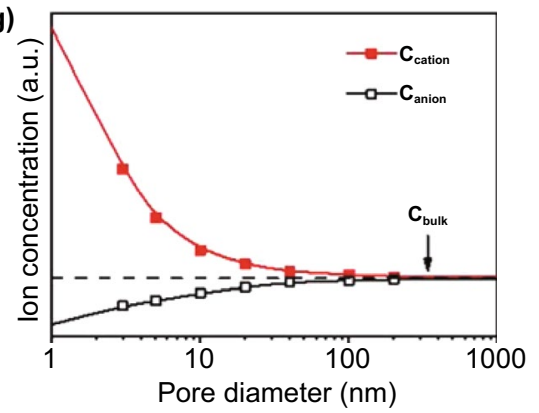

plating

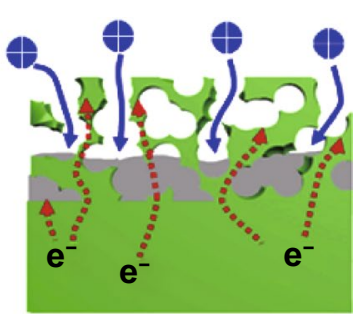

\section{Further Zn-plating}

(f) Locally concentrated electrolyte

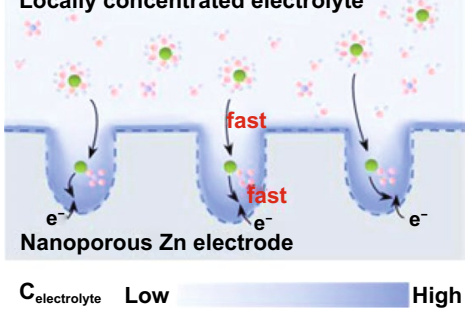

(h)

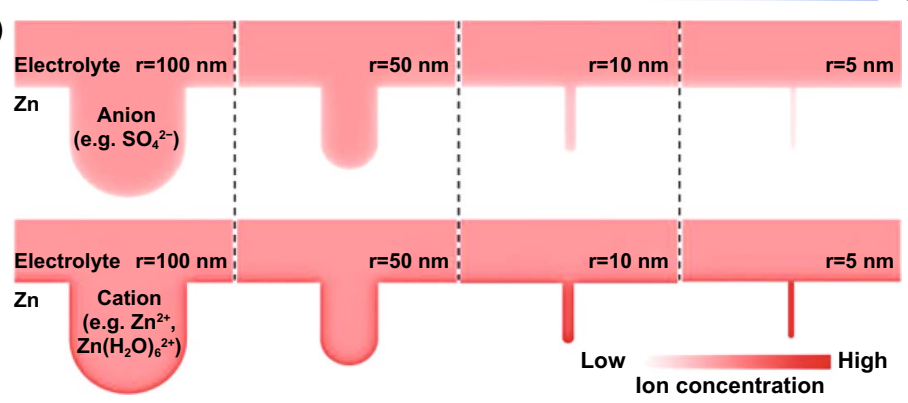

Fig.10 a Schematic illustrations of CM@CuO@Zn; b SEM images of Zn anode using reduced CM@CuO as the host with the capacity of $5 \mathrm{mAh} \mathrm{cm}{ }^{-2}$; c Schematic illustrations of the process of Zn deposition on CM@CuO and CM [97]. Copyright 2020, Wiley-VCH. d Schematic illustration of Zn deposition on the 3D Ni [98]. Copyright 2020, Wiley-VCH. e Stripping/plating performance of DCP-Zn-30 and pristine $\mathrm{Zn}$ foil cells with $0.1 \mathrm{mAh} \mathrm{cm}{ }^{-2}$ cutoff capacity at 3-10 mA cm${ }^{-2}$ [99]. Copyright 2020, Elsevier. f Nanoporous Zn electrode with interfacelocalized concentrated electrolyte; $\mathbf{g}$ The ion concentration at the electric double layer of nanoporous $\mathrm{Zn}$ metal with different pore diameters; $\mathbf{h}$ Surface charge densities of the cations and anions at the interface of $\mathrm{ZnSO}_{4}$ electrolyte and nanoporous $\mathrm{Zn}$ metal with different pore diameters ranging from 5 to $100 \mathrm{~nm}$ [100]. Copyright 2021, Elsevier 
(a)
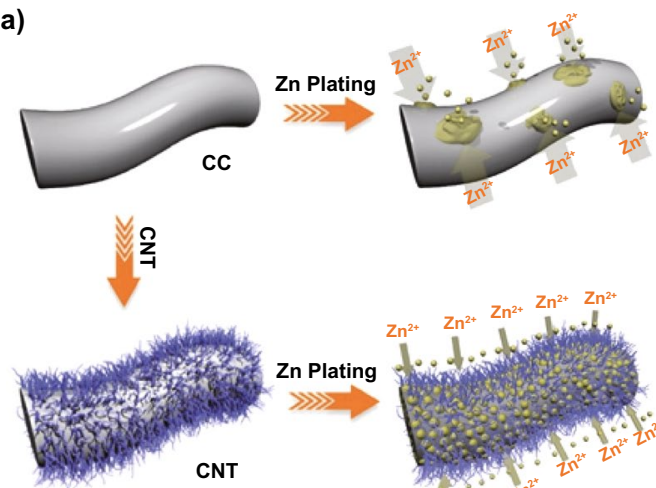

CNT
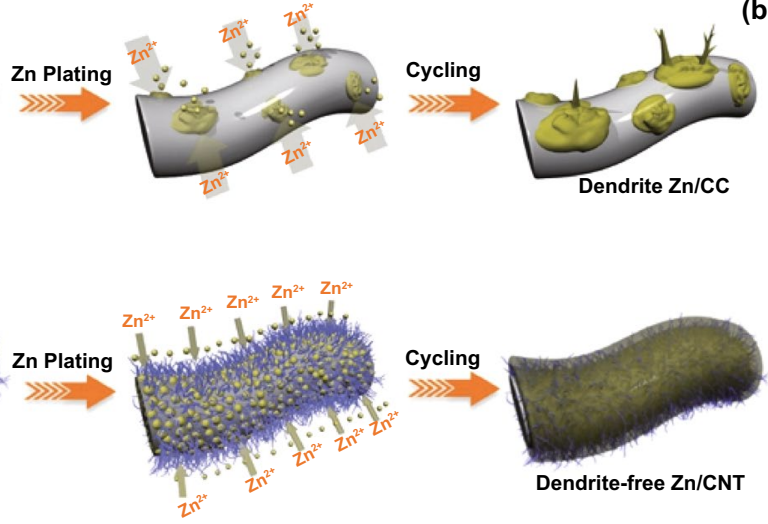

(b)

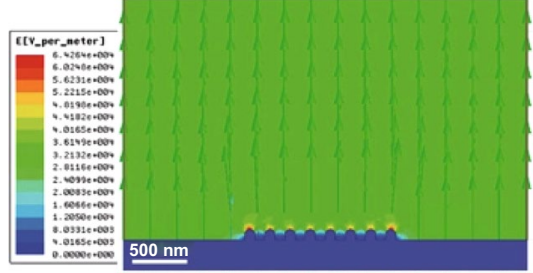

(c)

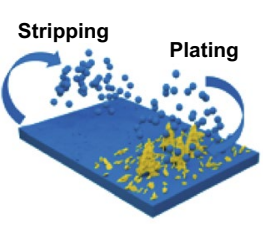

Bare Zn

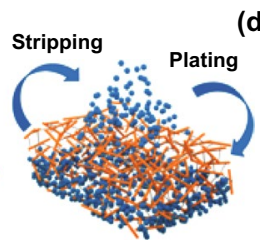

3D ZnP/CF (d)

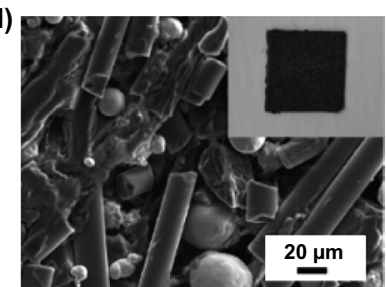

(e)

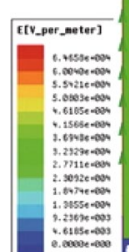

Cycling

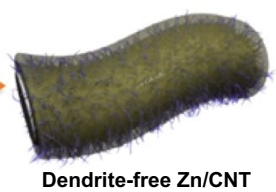

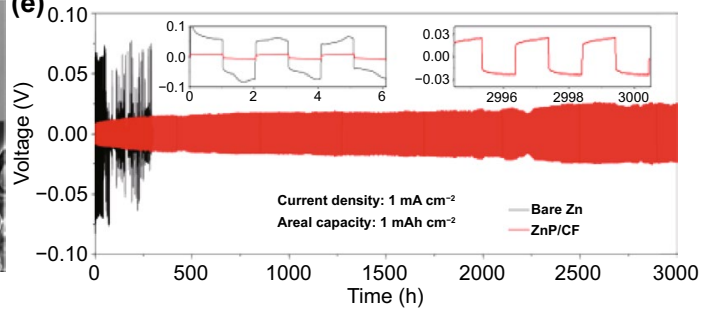

(f) 1

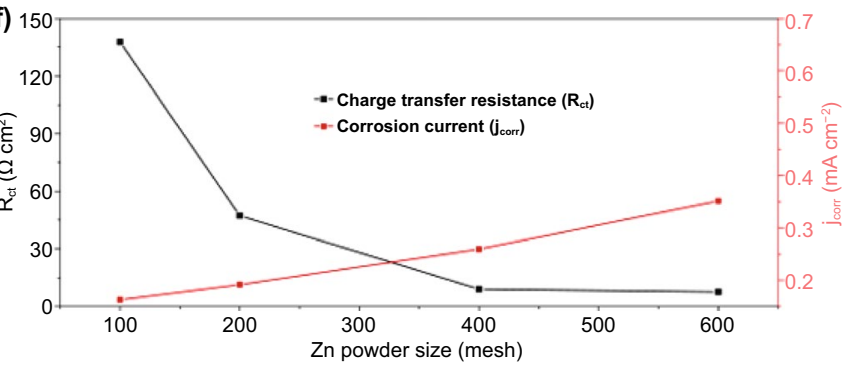

(g)

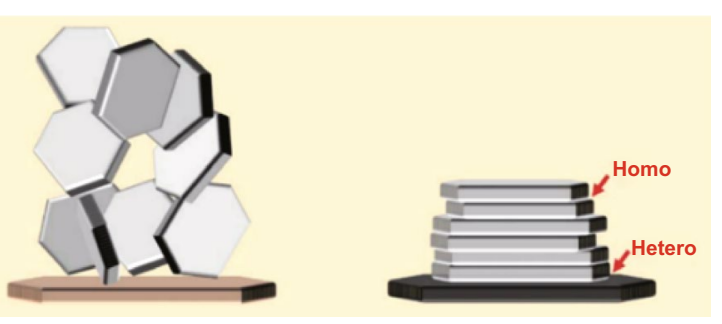

Fig. 11 a Schematic illustrations of Zn deposition on CC and CNT electrodes; $\mathbf{b}$ Models of the electric field distributions for a $\mathrm{Zn} / \mathrm{CC}$ electrode (top) and a Zn/CNT electrode (bottom) after Zn nuclei formation [106]. Copyright 2019, Wiley-VCH. c Schematic diagrams of the Zn growth mechanisms on different anode structures; $\mathbf{d}$ SEM image of $\mathrm{ZnP} / \mathrm{CF}$ composite electrode. Inset shows the optical pictures of ZnP/CF composite electrode; e Voltage profiles of the symmetric cells using $\mathrm{Zn}$ foil electrode and 3D ZnP/CF electrode at the current density of $1 \mathrm{~mA} \mathrm{~cm}^{-2}$ and $1 \mathrm{mAh} \mathrm{cm}^{-2}$; f Plots of the charge transfer resistance and the corrosion current with different particle sizes [107]. Copyright 2020, Elsevier. $\mathbf{g}$ Scheme illustrating the design principle of epitaxial metal electrodeposition [109]. Copyright 2019, American Association for the Advancement of Science

method of manipulating the anode host to control the preferential $\mathrm{Zn}$ deposition direction deserves further study [110].

In addition to metal-based and carbon-based anode hosts, there are other types of anode hosts, such as MOF-derived material [111]: ZIF-8-500, formed by annealing treatment at $500{ }^{\circ} \mathrm{C}$ and then undergoing a thermal reduction (Fig. 12a). On the one hand, the trace amount of $\mathrm{Zn}^{0}$ in the porous framework can serve as a uniform nucleus for subsequent $\mathrm{Zn}$ deposition, thus alleviating the problem of $\mathrm{Zn}$ dendrite growth. On the other hand, the high HER overpotential can effectively slow down water decomposition. With the assistance of ZIF-8-500, the CE of the anode was close to
$100 \%$, and the $\mathrm{Zn}$ iodine rechargeable battery exhibited a long life of 1,600 cycles (Fig. 12b). Moreover, due to the unique layered structure and high conductivity, MXene is a suitable material for the anode host. $\mathrm{Ti}_{3} \mathrm{C}_{2} \mathrm{~T}_{\mathrm{x}}$ MXene was proven to have the effect of regulating $\mathrm{Zn}$ deposition behavior (Fig. 12c, d) [112]. The increased specific surface area and enhanced hydrophilicity can ensure low current density and high ion mobility, so no $\mathrm{Zn}$ dendrites are observed after long charge-discharge cycles. It can be investigated that the layered structure of MXene can introduce a variety of zincophilic and chemically inert seed crystals as Zn nucleation sites to induce more uniform $\mathrm{Zn}$ deposition, which has 


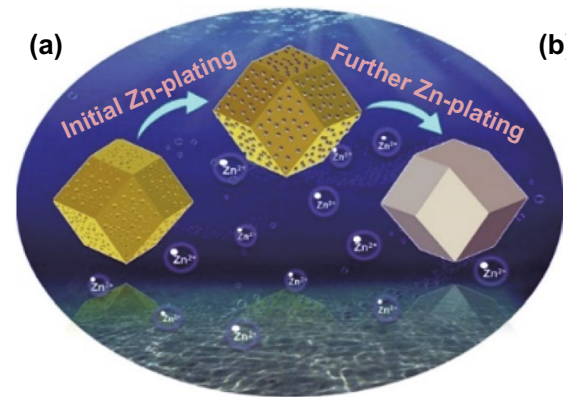

(b)
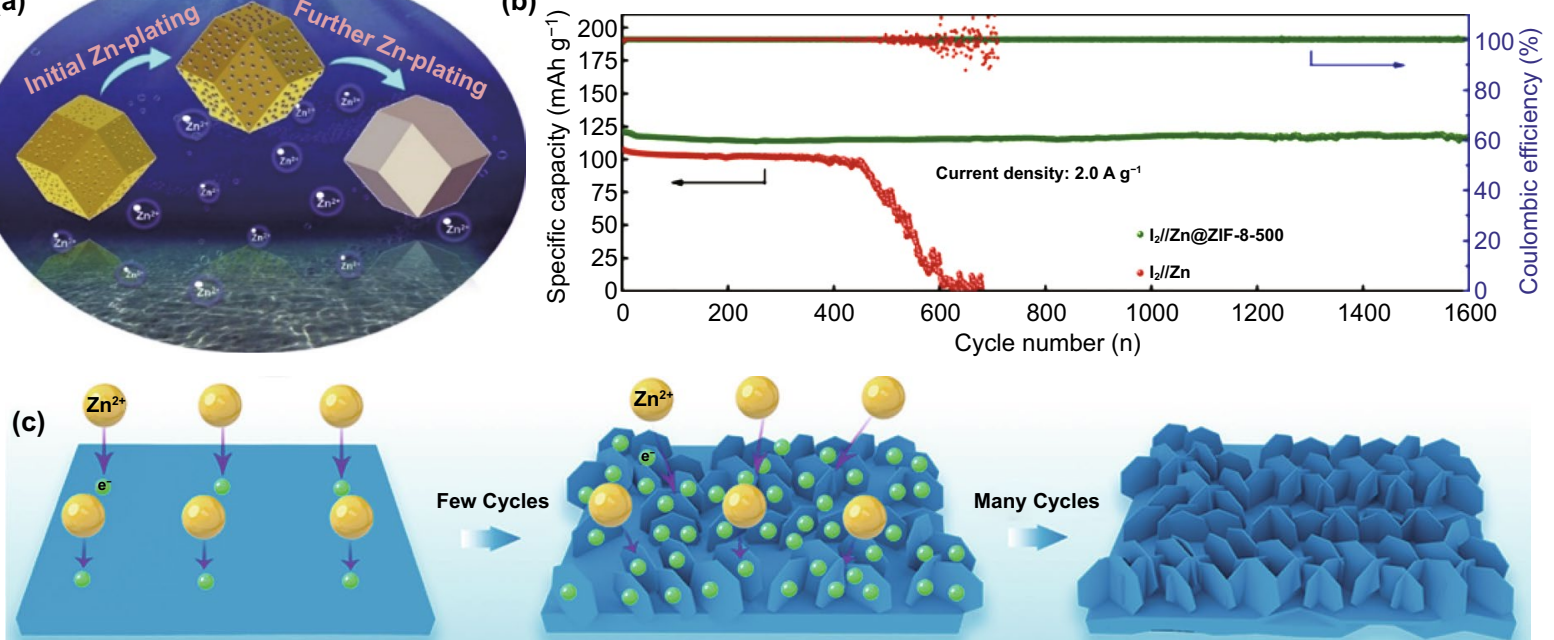

2D Planar Zn anode

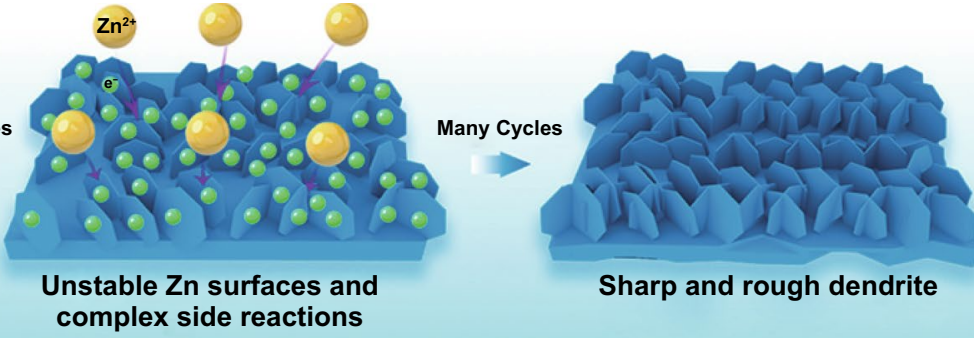

(d)

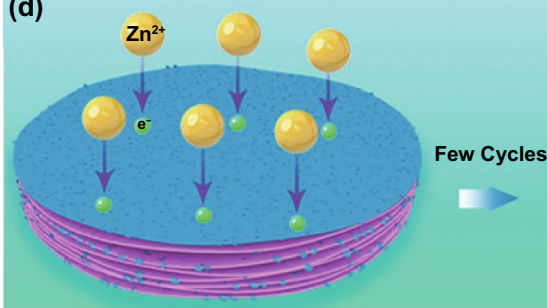

3D layered MXene@Zn anode

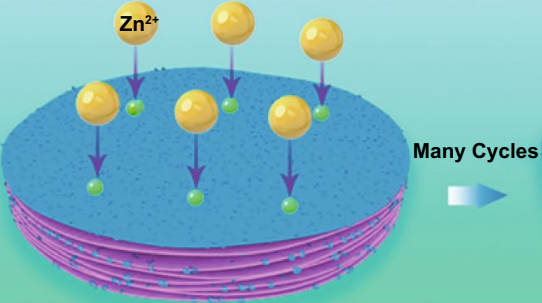

Fast electron and ion transport

Uniform Zn deposition

Fig. 12 a Schematic illustration of the Zn plating on the ZIF-8-500 electrode; $\mathbf{b}$ Electrochemical performances of the $\mathrm{I}_{2} / / \mathrm{Zn} @ \mathrm{ZIF}-8-500$ full

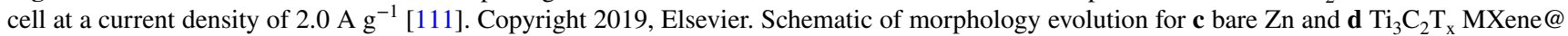
$\mathrm{Zn}$ anode during the stripping/plating process [112]. Copyright 2019, American Chemical Society

been similarly reported in the modification of Li metal-containing batteries $[113,114]$. Recently, it was reported that MXene paper was modified with antimony $(\mathrm{Sb})$ to be used as a $\mathrm{Zn}$ anode host. Benefiting from zincophilic Sb seeds and MXene architecture, stable battery performance can be achieved. Similarly, $\mathrm{Cu}$ or $\mathrm{Ni}$ can replace $\mathrm{Sb}$ as a seed crystal to modify MXene paper [115].

\subsection{Alloying Anode}

Alloy refers to mixing metal with other metals or nonmetals to obtain a material with metallic properties. $\mathrm{Zn}$ can be fused with various elements to form $\mathrm{Zn}$-based alloys, classified into binary alloys, ternary alloys, and multi-element alloys according to the number of their components. In addition to Ga-In-Zn [116, 117] and Zn-Sn-Pb [118] alloys, the Zn alloys currently reported on mild aqueous $\mathrm{Zn}$ anodes are mainly binary alloys, such as $\mathrm{Cu}-\mathrm{Zn}[89,119,120], \mathrm{Ag}-\mathrm{Zn}$ [89, 121-123], Zn-Al [124], Zn-Mn [125], Zn-Sb [115], and Zn-P [69]. The interaction between alloy components will form an alloy phase with a specific structure and composition, which can be divided into solid solution and intermetallic compound [126]. Even though the component species are the same, the alloy properties may differ due to different alloy phases formed. This greatly enriches the categories of alloys with other properties. The electrochemical alloying reaction of $\mathrm{Zn}$ includes a restructuring reaction and solid solution reaction [127]. Compared with the former, the latter does not require a significant phase change, resulting in less demand for additional activation energy, which means lower charge/discharge voltage hysteresis. Instead of standard $\mathrm{Zn}$ stripping/plating on the metal foil surface, the dealloying/ alloying reactions involved in the solid solution reaction should be the mechanism that indicates the inward-transfer 
and reversible extraction of $\mathrm{Zn}$ atoms in the $\mathrm{Zn}$ alloy [128]. Therefore, based on the stable solution reaction, the $\mathrm{Zn}$ alloy anode with different alloy phases can significantly enhance the battery performance.

The addition of electrochemically inert metals not only changes the physical properties but also enhances electrochemical performance. Reviewing the $\mathrm{Zn}$ alloy anode, $\mathrm{Cu}$ and $\mathrm{Ag}$ species can improve the corrosion resistance of $\mathrm{Zn}$ alloys, and the corrosion potential is close to that of the pure metal introduced (Fig. 13a) [18]. However, this conclusion is affected by many factors, such as element ratio, microstructure, and the alloy phase, making it difficult to explain the detailed corrosion resistance mechanism [18, 24]. A relatively simple strategy has been proposed that the enhanced corrosion resistance of alloys can be analyzed from the perspective of energy supported by DFT (Fig. 13b) [121].
Comparing the minimum energy cost of removing $\mathrm{Zn}$ atoms from pure $\mathrm{Zn}$ and $\mathrm{Zn}_{0.5} \mathrm{Ag}_{0.5}, 1.10 \mathrm{eV}$ for pure $\mathrm{Zn}$ with 100 crystal plane is lower than $1.40 \mathrm{eV}$ for $\mathrm{Zn}_{0.5} \mathrm{Ag}_{0.5}$ with a 001 crystal plane, implying that the $\mathrm{Zn}$ atoms in $\mathrm{Zn}_{0.5} \mathrm{Ag}_{0.5}$ have lower reactivity. The more energy cost for $\mathrm{Zn}$ stripping from the $\mathrm{Zn}_{\mathrm{x}} \mathrm{Ag}_{1-\mathrm{x}}$ alloy corresponds to a higher redox potential (Fig. 13c); thus, the $\mathrm{Zn}_{\mathrm{x}} \mathrm{Ag}_{1-\mathrm{x}}$ alloy has better corrosion resistance. Similarly, the alloy's suppression strategy for dendrites can also be analyzed from the nucleation energy. Compared with pure $\mathrm{Zn}$ metal, $\mathrm{Zn}_{\mathrm{x}} \mathrm{Ag}_{1-\mathrm{x}}$ alloys have a lower nucleation energy barrier (Fig. 13c). This indicates that after $\mathrm{Zn}$ is reduced, it will spontaneously form an alloy phase with Ag lattice through solid solution reaction, rather than accumulating at sites containing only Zn species (Fig. 13d) [89, 121, 122]. However, excessive $\mathrm{Zn}$ deposition will still form a $\mathrm{Zn}$ layer on the alloy surface, inducing dendrite growth.
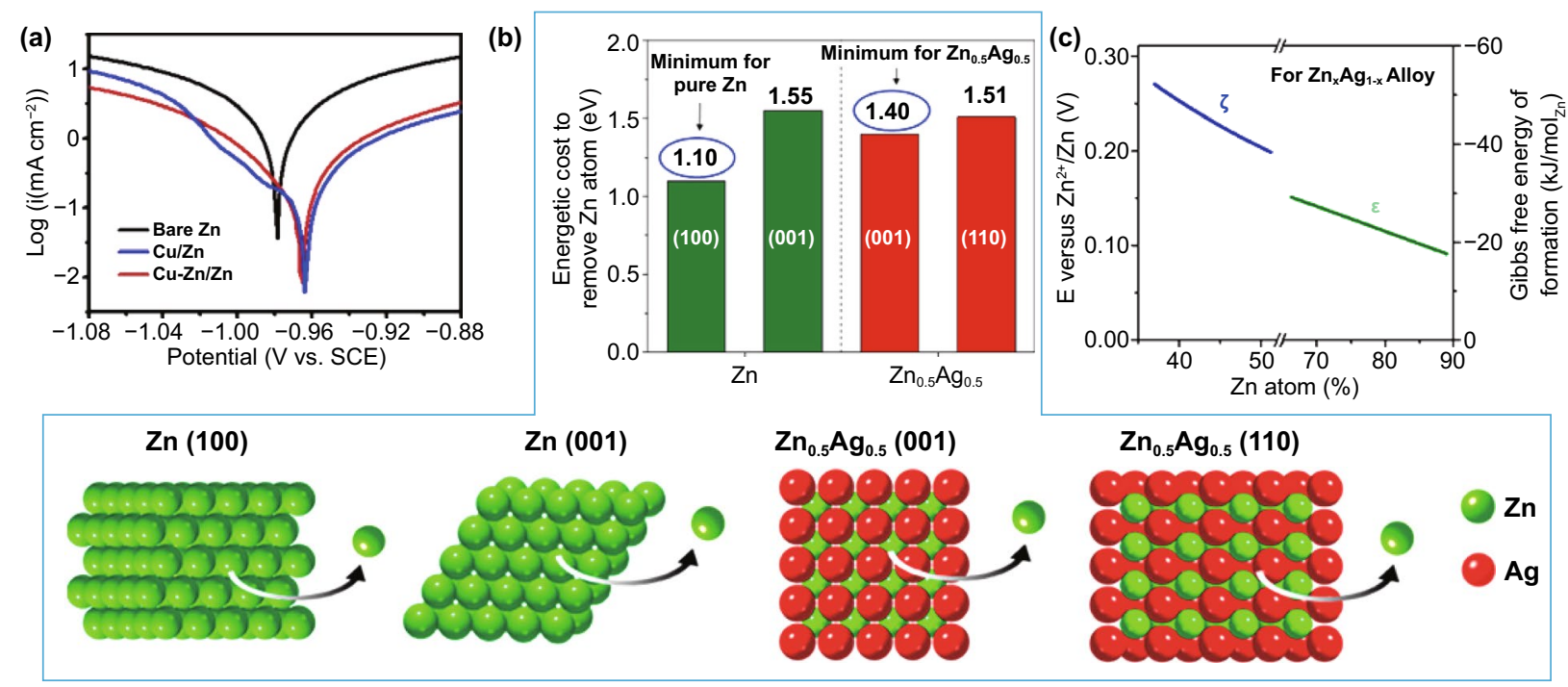

(d)

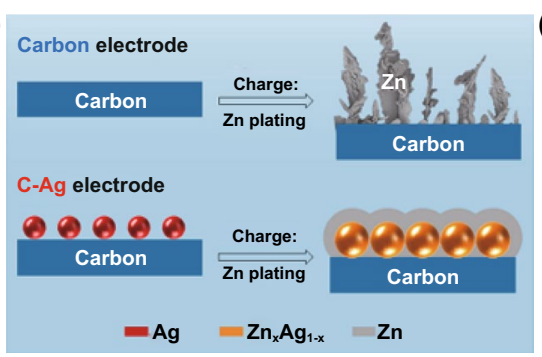

(e)

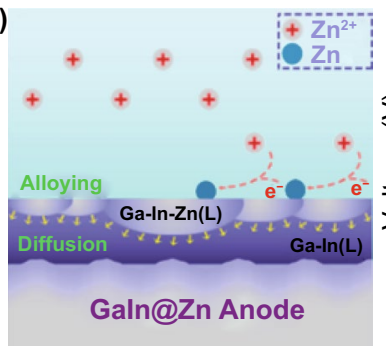

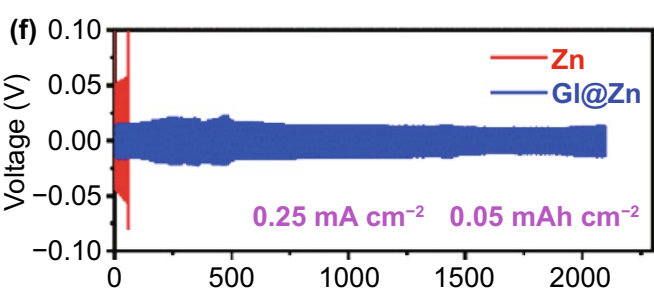

Fig. 13 a Linear polarization curve of $\mathrm{Cu} / \mathrm{Zn}$ and $\mathrm{Cu}-\mathrm{Zn} / \mathrm{Zn}$ electrode in $3 \mathrm{M} \mathrm{ZnSO}_{4}$ electrolyte [119]. Copyright 2020, Elsevier. b DFT simulation results showing the energetic cost of removing a $\mathrm{Zn}$ atom from the pure $\mathrm{Zn}$ metal and $\mathrm{Zn}_{0.5} \mathrm{Ag}_{0.5}$ alloy. Constructed models: $\mathrm{Zn}$ with 001 and 100 surfaces; $Z_{0.5} \mathrm{Ag}_{0.5}$ with 110 and 001 surfaces; c Calculated Gibbs free energy of formation at room temperature of $\mathrm{Zn}$, $\zeta$ - and $\varepsilon-\mathrm{Zn}_{\mathrm{x}} \mathrm{Ag}_{1-\mathrm{x}}$ alloy phases and the corresponding electrochemical potential shift of $\mathrm{Zn}^{2+} / \mathrm{Zn}_{\mathrm{x}} \mathrm{Ag}_{1-\mathrm{x}}$ compared with that of $\mathrm{Zn}^{2+} / \mathrm{Zn} \mathbf{d} \mathrm{Schematic}$ of $\mathrm{Zn}$ deposition on the (top) carbon paper substrate and (bottom) carbon paper slurry coated with Ag nanoparticles [121]. Copyright 2021, American Chemical Society. e Dendrite-free GaIn@Zn anode by alloying-diffusion synergistic strategy; f Voltage profiles of symmetric cells using bare Zn foil and GaIn@Zn at a current density of $0.25 \mathrm{~mA} \mathrm{~cm}^{-2}$ [116]. Copyright 2021, American Chemical Society 
Differently, liquid alloys have high fluidity and deformability and can avoid the formation of dendrites through a selfhealing mechanism $[116,117]$. The high $\mathrm{Zn}$ affinity of liquid Ga-In alloy enables the preferential formation of $\mathrm{Ga}-\mathrm{In}-\mathrm{Zn}$ ternary alloy (Fig. 13e). When the $\mathrm{Zn}$ species in the alloy reaches saturation, excess $\mathrm{Zn}$ spontaneously accumulates under the alloy layer instead of on the surface. Thus, the alloy surface can be maintained smooth, and dendrites cannot be formed on the alloy surface. Collaborating with the high HER overpotential of the alloy and the low impedance of the liquid-liquid interface, the symmetrical battery assembled by the gain anode can be cycled stably for $2000 \mathrm{~h}$ with a capacity of $0.05 \mathrm{mAh} \mathrm{cm} \mathrm{cm}^{-2}$ at a current density of $0.25 \mathrm{~mA} \mathrm{~cm}^{-2}$ (Fig. 13f).

Based on the above analysis, it can be concluded that the alloy mainly suppresses side reactions and dendrite growth by increasing the HER overpotential and reducing the nucleation energy barrier. However, $\mathrm{Zn}$ alloys with more active metals than $\mathrm{Zn}$ may suffer from the competitive reaction of metal species and corrosion problems, which means that other compensable properties are needed to serve stable $\mathrm{Zn}$ anode. It has been proposed that the eutectic $\mathrm{Zn}_{88} \mathrm{Al}_{12}$ alloy with alternating $\mathrm{Al}$ and $\mathrm{Zn}$ layers can induce uniform $\mathrm{Zn}$ deposition by spontaneously constructing an insulating frame, which can be detailed from the two stages of $\mathrm{Zn}$ stripping and plating (Fig. 14a) [124]. In the initial stripping stage, since the standard equilibrium potential of $\mathrm{Al}^{3+} / \mathrm{Al}$ $(-1.66 \mathrm{~V})$ is lower than that of $\mathrm{Zn}^{2+} / \mathrm{Zn}$, Al will react in preference to $\mathrm{Zn}$ and be converted to $\mathrm{Al}_{2} \mathrm{O}_{3}$. Subsequently, the dense and insulating $\mathrm{Al}_{2} \mathrm{O}_{3}$ passivation layer protects the anode from further oxidation so that the Al-Zn alloy anode can maintain stability. During the plating process, the positive electrostatic shielding layer formed around the plate prevents the $\mathrm{Zn}$ nucleation on the surface of $\mathrm{Al}_{2} \mathrm{O}_{3}$, thereby guiding $\mathrm{Zn}$ deposition in the correct position.

It is worth noting that the alloy preparation approaches in the laboratory mainly involve electrodeposition and chemical replacement [126], which results in a relatively single appearance of the alloy. Different microstructures of alloys containing the same element species have other effects on anode performance. By developing a reasonable preparation process, the alloy can be designed into a favorable structure to further enhance the performance of the anode. Impressively, a co-electrodeposition process of various ions has been proposed. The evolution of $\mathrm{H}_{2}$ bubbles at the solid-liquid interface can lead to the formation of a 3D anode structure [125]. Applying this strategy, the cauliflower-like $3 \mathrm{D} \mathrm{Zn}_{3} \mathrm{Mn}$ alloy can be obtained in the solution containing $\mathrm{Zn}^{2+}$ and $\mathrm{Mn}^{2+}$ ions (Fig. 14b) [125]. The porous morphology with a large specific surface area promotes effective $\mathrm{Zn}^{2+}$ ion migration. Additionally, due to the relatively high binding energy on the alloy surface, $\mathrm{Zn}$ nucleation and growth are induced and regulated (Fig. 14c). During the $\mathrm{Zn}$ deposition process, the deposition rate in the trenches is much larger than that in the protruding area, which minimizes the formation of dendrites (Fig. 14d-f). Benefiting from these advantages, the $\mathrm{Zn}_{3} \mathrm{Mn}$ alloy anode has ultra-high reversibility. Even in the seawater-based electrolyte, despite the interference of various impurity ions, it still shows high stability and reliability (Fig. 14g). Note that this co-electrodeposition strategy can be extended to other alloy systems by adjusting the composition of the deposition solution, the applied deposition current or voltage, and the deposition time. Therefore, it is recommended that while introducing more element species to the alloy anode, the alloy structure can be designed by developing the alloy preparation process.

\subsection{Intercalation Anode}

The successful application of intercalated anodes in lithium batteries has attracted more and more attention. Unlike the direct deposition/stripping of charged ions on the conventional metal anode, the lithium ions participate in battery storage and release of energy through intercalation/deintercalation on intercalation anodes [129]. Since there is no metal electrode, dendrite growth and corrosion are eliminated. This unique anode structure and its working mechanism have also inspired people to explore the application of intercalation anodes in mild aqueous ZIBs. However, there are few reports on intercalated $\mathrm{Zn}$ anodes, and the properties of the proposed materials are generally unsatisfactory. Due to the lack of high-performance materials and the ambiguity of the $\mathrm{Zn}$ storage mechanism, low energy density is one of the biggest challenges of intercalation anodes. The intercalation electrode materials that have been reported are mainly collected in Chevrel phases, transition metal oxides or sulfides, complex transition metal compounds, organic compounds, etc. [130]. There have been some reviews detailing the development of intercalation electrodes in mild aqueous electrolytes. Based on this status 
(a)
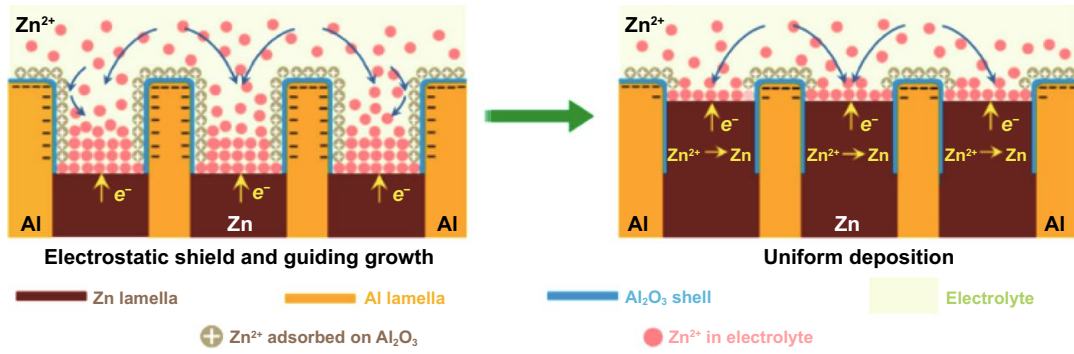

(c)
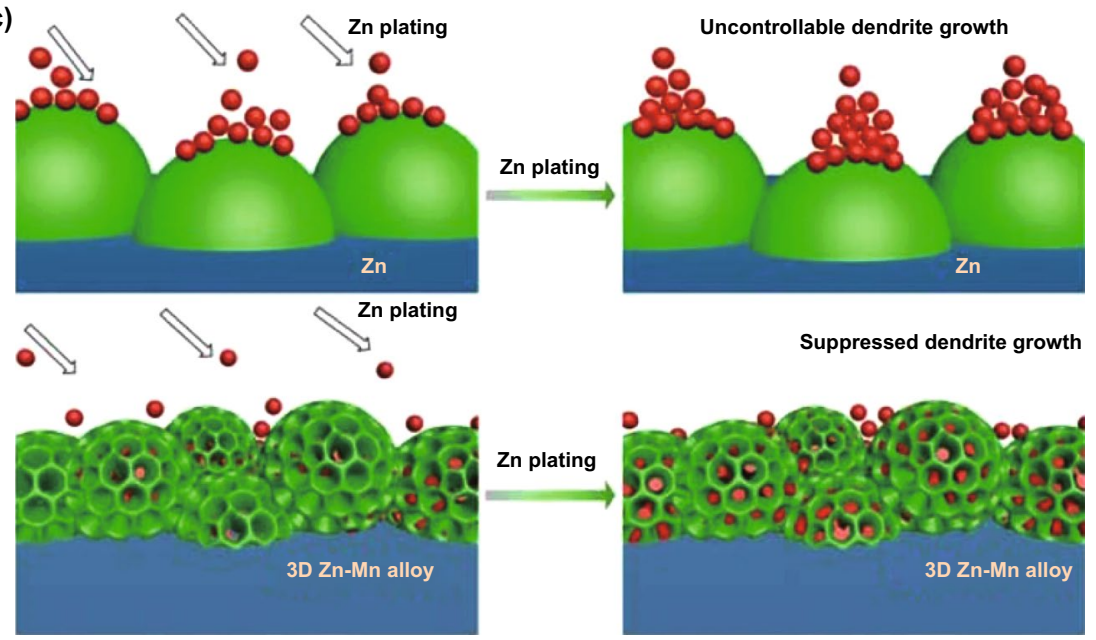

$\mathrm{Zn}^{2+}$ in electrolyte

(b)

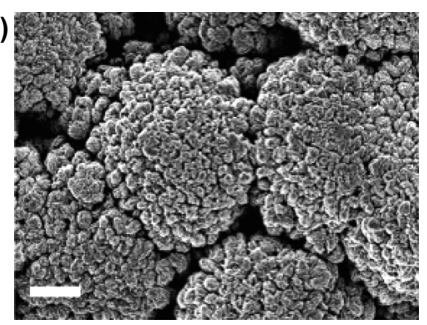

(d)

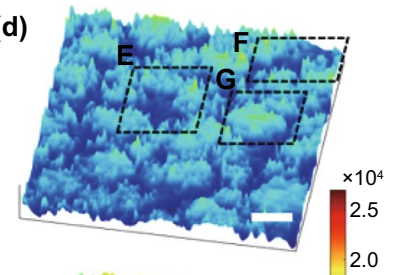

(e)

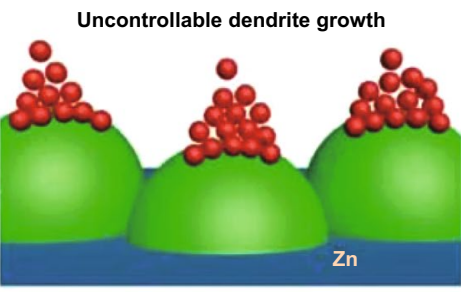

Suppressed dendrite growth
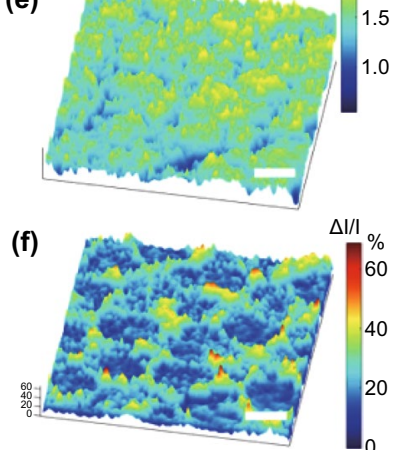

(g)

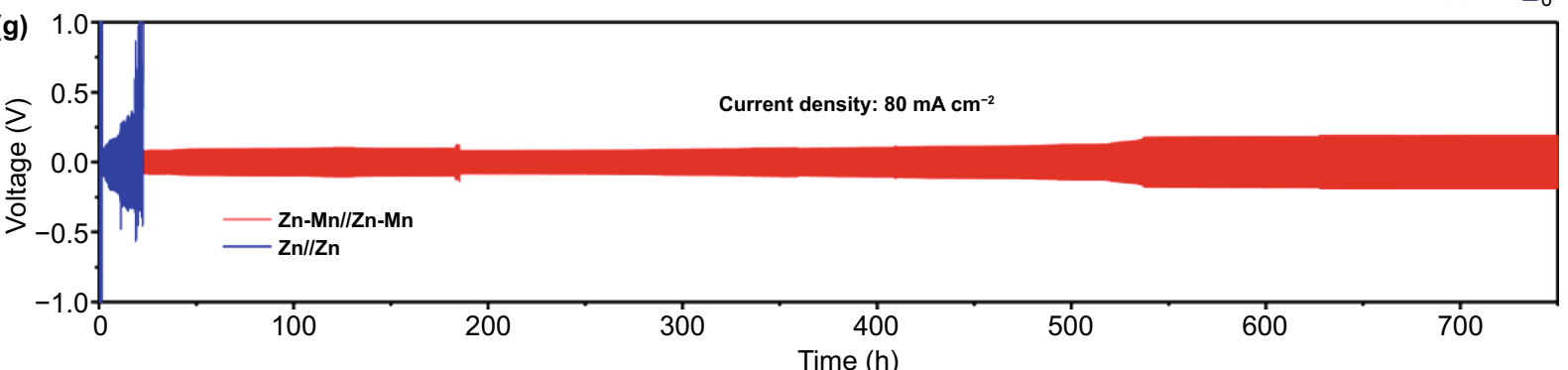

Fig. 14 a Schematic illustrations of eutectic Al-Zn for uniform Zn deposition [124]. b SEM image of Zn-Mn alloy; c Schematic illustration of $\mathrm{Zn}$ plating processes on $\mathrm{Zn}$ anode and $\mathrm{Zn}-\mathrm{Mn}$ anode; The images of $3 \mathrm{D} \mathrm{Zn}-\mathrm{Mn}$ alloy by in situ optical microscope before $\mathbf{d}$ and after $\mathbf{e} \mathrm{Zn}$ plating, $\mathbf{f}$ was calculated by $(\mathrm{d}-\mathrm{e}) / \mathrm{e}=(\Delta \mathrm{I} / \mathrm{I}) ; \mathbf{g}$ Long-term galvanostatic cycling performance of symmetric $\mathrm{Zn}-\mathrm{Mn}$ and pristine $\mathrm{Zn}$ cells at a current density of $80 \mathrm{~mA} \mathrm{~cm}^{-2}$ (areal capacity, $16 \mathrm{mAh} \mathrm{cm}^{-2}$; electrolyte, $2 \mathrm{M} \mathrm{ZnSO}_{4}$ in seawater) [125]

quo, recently, a high-rate intercalation anode (PTCDI/rGO) could be obtained by hybridizing perylene-3,4,9,10-tetracarboxylic diimide (PTCDI) and reduced graphene oxide (rGO) (Fig. 15a) [131]. During the charge and discharge, the PTCDI organic electrode provides high electron mobility and prevents the dissolution of discharge products. Besides, the porous structure caused by rGO maintains a wide range of active sites and ion diffusion paths. In addition, Yang et al. [132] proposed mixed-valence $\mathrm{Cu}_{2-\mathrm{x}} \mathrm{Se}$ as an intercalation anode to solve the problems of $\mathrm{Zn}$ dendrite growth and electrolyte decomposition (Fig. 15b-e). Stable material structure, abundant cation sites, and high conductivity ensure the rapid insertion and extraction of $\mathrm{Zn}^{2+}$ ions. The low-valence copper in the electrode material is not only conducive to the generation of suitable intercalation formation energy (Fig. 15f) but also can reduce the $\mathrm{Zn}^{2+}$ ion diffusion barrier (Fig. 15g). Low-valence copper can regulate the active sites of $\mathrm{Zn}^{2+}$ ion storage and optimize the electronic interaction between active sites and intercalated $\mathrm{Zn}^{2+}$ ions. 
(a)

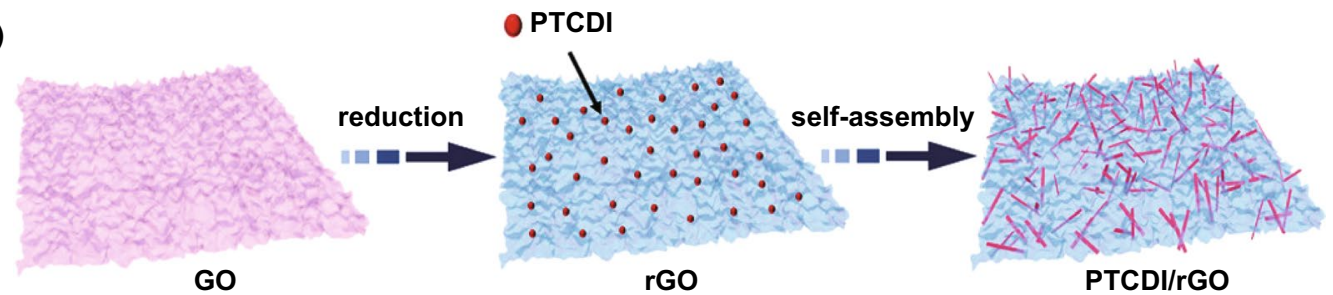

(b)
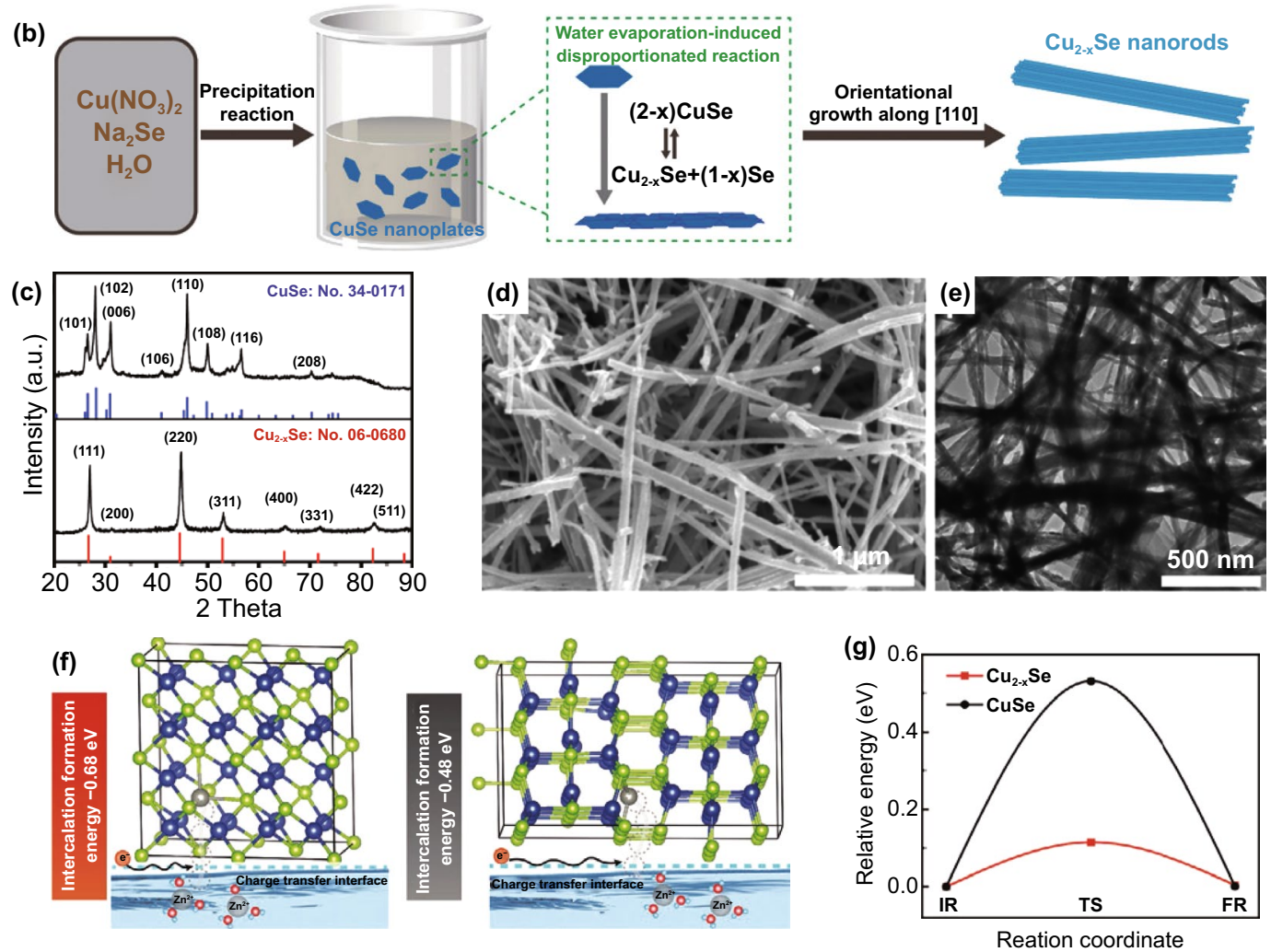

Fig. 15 a Schematic illustration of preparing the PTCDI/rGO composite [131]. b Schematic illustration of the formation of the Cu $\mathrm{u}_{2-\mathrm{x}} \mathrm{Se}$ nanorods; $\mathbf{c}$ XRD patterns of $\mathrm{Cu}_{2-x} \mathrm{Se}$ and CuSe; SEM $\mathbf{d}$ and TEM e images of the $\mathrm{Cu}_{2-\mathrm{x}} \mathrm{Se}$ nanorods; $\mathbf{f}$ Charge transfer process at the reaction interface and intercalation formation energy of $\mathrm{Cu}_{2-x} \mathrm{Se}$ (right) and CuSe (left); $\mathbf{g}$ Diffusion barrier of $\mathrm{Zn}^{2+}$ ion in $\mathrm{Cu}_{2-\mathrm{x}} \mathrm{Se}$ and $\mathrm{CuSe}$ [132]. Copyright 2020, Wiley-VCH

When matched with $\mathrm{Zn}_{\mathrm{x}} \mathrm{MnO}_{2}$, the full battery exhibited an extremely long cycle life of over 20,000 cycles at $2 \mathrm{~A} \mathrm{~g}^{-1}$.

\subsection{Liquid Electrolyte}

As the medium for conducting ions between the anode and cathode, the liquid aqueous electrolyte profoundly affects anode performance in mild aqueous ZIBs. A variety of modified liquid electrolytes have been proposed, which reflect different anode control strategies. This section mainly discusses the regulation strategies of liquid electrolytes on
$\mathrm{Zn}$ deposition behavior, including weakening of solvation effect, suppression of 2D diffusion, formation of electrostatic shielding layer, and formation of in situ SEI layer.

\subsubsection{Weakening of Solvation Effect}

As mentioned above, in a mild electrolyte, $\mathrm{Zn}^{2+}$ ions can cooperate with water molecules to form $\left[\mathrm{Zn}\left(\mathrm{H}_{2} \mathrm{O}\right)_{6}\right]^{2+}$ with a sheath structure, the bulky solvation structure is not conducive to the migration and deposition of $\mathrm{Zn}^{2+}$ ions, resulting in reduced battery performance (Fig. 16a). It is necessary to 
(a)

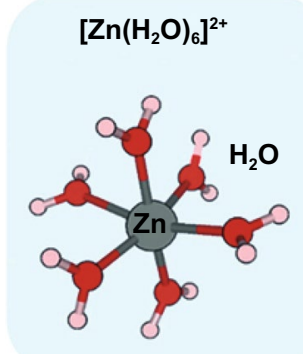

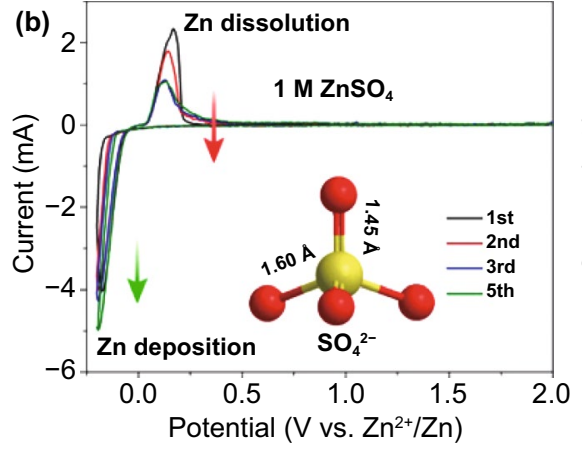

(e)

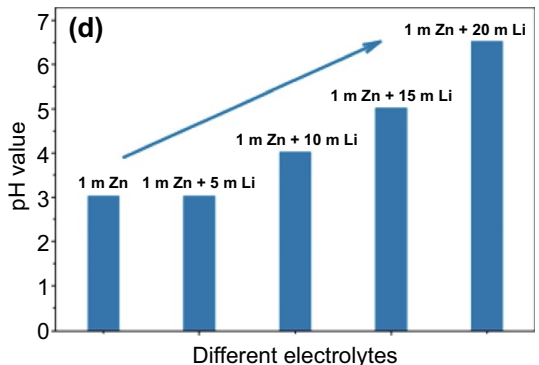

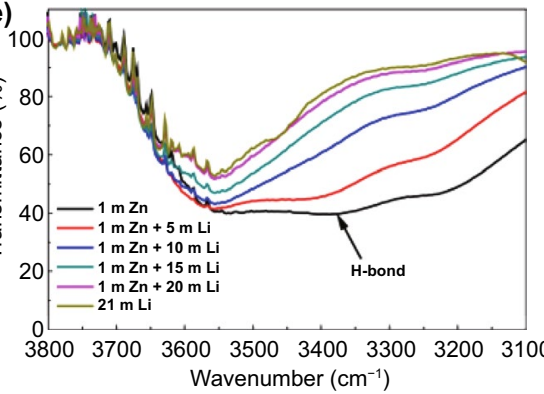
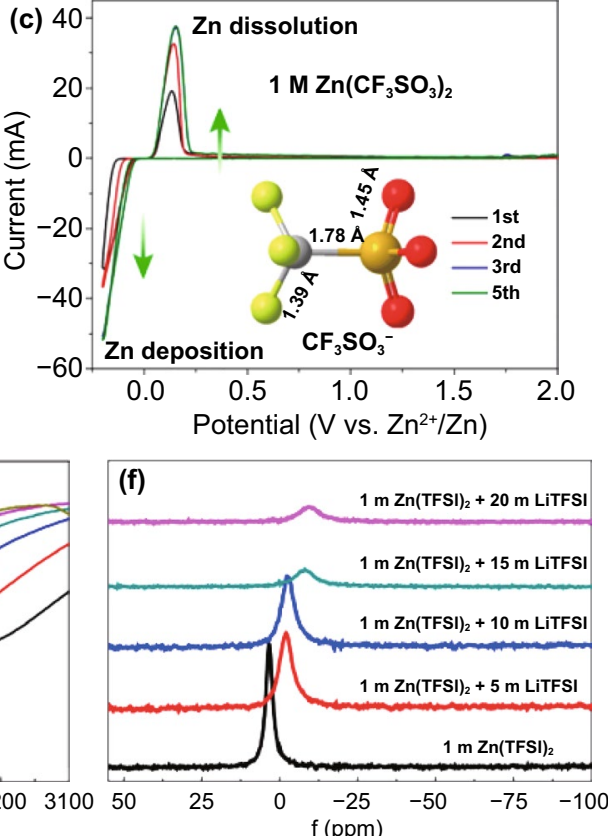
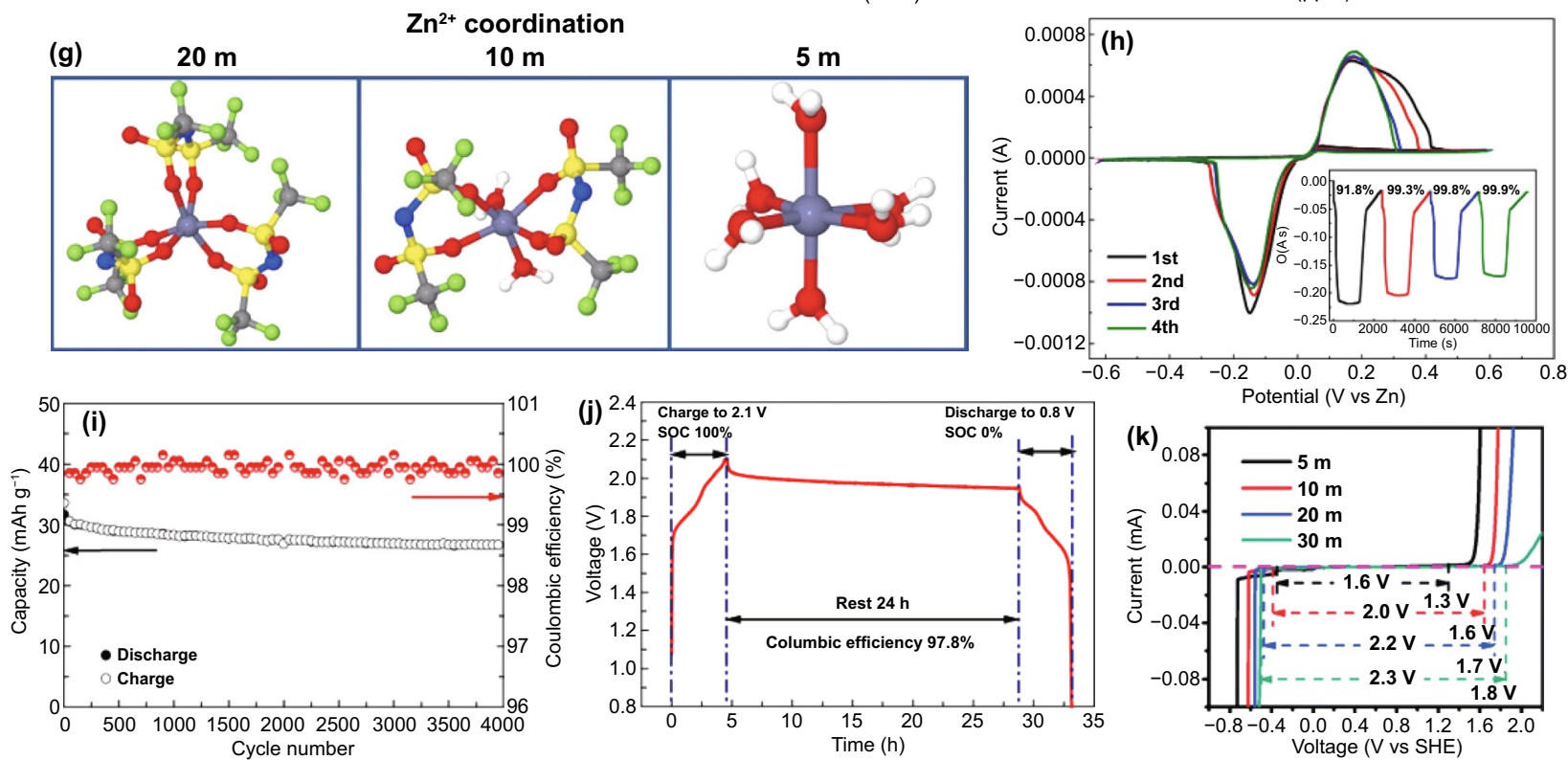

Fig. 16 a Coordination environment of $\mathrm{Zn}^{2+}$ in water [54]. Copyright 2020, Wiley-VCH. Cyclic voltammograms of $\mathrm{Zn}$ electrode in aqueous electrolyte of $\mathbf{b} 1 \mathrm{M} \mathrm{Zn}\left(\mathrm{CF}_{3} \mathrm{SO}_{3}\right)_{2}$ and $\mathbf{c} 1 \mathrm{M} \mathrm{ZnSO}_{4}$ at the scan rate of $0.5 \mathrm{mV} \mathrm{s}^{-1}$ between -0.2 and $2.0 \mathrm{~V}$ [133]. Copyright 2016, American Chemical Society. d The $\mathrm{pH}$ values of the electrolytes with varying LiTFSI concentrations; $\mathbf{e}$ The progression of FTIR spectra with salt concentration between 3,800 and $3,100 \mathrm{~cm}^{-1}$; f The change with salt concentration of chemical shifts for ${ }^{17} \mathrm{O}$ nuclei in solvent (water); $\mathbf{g}$ Representative $\mathrm{Zn}^{2+}$ solvation structures in the electrolytes with $\left.1 \mathrm{M} \mathrm{Zn(TFSI}\right)_{2}$ and three concentrations of LiTFSI (5, 10, and $\left.20 \mathrm{M}\right)$; h Cyclic voltammogram of $\mathrm{Zn}$ plating/stripping in a three-electrode cell using a Pt disk ( $2 \mathrm{~mm}$ in diameter) as the working and $\mathrm{Zn}$ as the reference and counter electrodes at a scan rate of $1 \mathrm{mV} \mathrm{s}^{-1}$. Inset: chronocoulometry curves; i Cycling stability and CE of the $\mathrm{Zn} / \mathrm{LiMn}_{2} \mathrm{O}_{4}$ full cell in $\mathrm{HCZE}$ at $4 \mathrm{C}$ rates; j Storage performance evaluated by resting for $24 \mathrm{~h}$ at $100 \%$ state of charge (SOC) after ten cycles at $0.2 \mathrm{C}$, followed by full discharging [134]. Copyright 2018, Springer Nature. k Electrochemical stability window of the $\mathrm{ZnCl}_{2}$ electrolyte at different concentrations [135]. Copyright 2018, Royal Society of Chemistry 
reduce the degree of solvation. There are various influencing factors that affect the solvation structure of $\mathrm{Zn}^{2+}$ ion in liquid aqueous electrolyte, such as species of anion salt, electrolyte concentration, and additives.

Generally, in Zn salt electrolytes, anions may affect the solvation process of $\mathrm{Zn}^{2+}$ ions. The commonly reported anion salt species currently mainly involve $\mathrm{SO}_{4}{ }^{2-}, \mathrm{CF}_{3} \mathrm{SO}_{3}{ }^{-}$, TFSI ${ }^{-}, \mathrm{CH}_{3} \mathrm{COO}^{-}, \mathrm{NO}_{3}{ }^{-}, \mathrm{Cl}^{-}$, etc. [52]. Although the most widely used $\mathrm{SO}_{4}{ }^{2-}$ anion has a stable structure and excellent compatibility with $\mathrm{Zn}$ anodes, it cannot effectively alleviate the solvation effect, which has become resistant to further development. It has been reported that in the $\mathrm{Zn}\left(\mathrm{CF}_{3} \mathrm{SO}_{3}\right)_{2}$ electrolyte, the bulky $\mathrm{CF}_{3} \mathrm{SO}_{3}{ }^{-}$anions with a single charge can reduce the number of water molecules in the solvent sheath around the $\mathrm{Zn}^{2+}$ ion, and the desolvated $\mathrm{Zn}^{2+}$ ion can achieve faster transfer, thereby increasing the $\mathrm{Zn}^{2+}$ ion migration and charge transfer rate (Fig. 16b, c) [133]. Therefore, a battery with a reduced solvation effect can be obtained by rationally selecting the anion species of the electrolyte.

Increasing the salt concentration in the electrolyte to reduce the contact chance of $\mathrm{Zn}^{2+}$ ions with the surrounding water seems to solve this problem effectively. As a proof of concept, Wang et al. [134] found that as the concentration of LiTFSI increases, the $\mathrm{pH}$ of the electrolyte gradually increases and finally stabilizes at about 7 , which meant that the interaction between water and $\mathrm{Zn}^{2+}$ ions was inhibited (Fig. 16d). According to Fourier transform infrared (FTIR) spectra (Fig. 16e), the $3414 \mathrm{~cm}^{-1}$ peak, which reflected the hydrogen bond, disappeared completely at a salt concentration of $10 \mathrm{M}$, indicating that the hydrogen bond network in the water had been extensively destroyed. This phenomenon had also been confirmed in NMR (nuclear magnetic resonance) spectra (Fig. 16f). Based on molecular dynamics (MD) simulation, it could be recognized that ultra-high-concentration electrolytes containing $1 \mathrm{M} \mathrm{Zn(TFSI})_{2}$ and $20 \mathrm{M}$ LiTFSI completely changed the coordination environment of $\mathrm{Zn}^{2+}$ ions, in which $\mathrm{Zn}^{2+}$ ions only coordinated with TFSI ${ }^{-}$, while water molecules were surrounded by TFSI $^{-}$(Fig. 16g). At the same time, the high concentration of electrolytes reduces water activity and water-induced side reactions. Hydrogen evolution and corrosion were dramatically suppressed by eliminating the step of desolvation and blocking the contact of water with the anode interface. The assembled battery not only exhibited high $\mathrm{CE}$ in the electrochemical test but also had a high-capacity retention rate during storage (97.8\% after 24 h) (Fig. 16h-j). Similarly, Zhang et al.
[135] did not observe the formation of anode by-products in the "water-in-salt" electrolyte with $30 \mathrm{M} \mathrm{ZnCl}_{2}$, in which the solvated structure will be converted to $\left[\mathrm{ZnCl}_{4}\right]^{2-}$. The electrochemical stability window of the $\mathrm{ZnCl}_{2}$ electrolyte was widened along with the decrease in the hydrogen evolution potential due to the increase in concentration, which improved the CE of Zn plating/stripping as well (Fig. 16k). However, in addition to the increasing cost and reducing battery energy density, the electrolyte with an excessively high concentration exposes the characteristics of high viscosity, poor wettability, and low ionic conductivity, which limits the commercial development of $\mathrm{Zn}^{2+}$ ion batteries. Nevertheless, this strategy of applying high-concentration electrolytes still has a great practical effect. For example, appropriately increasing the electrolyte concentration is beneficial to the improvement in battery performance. We need to explore the appropriate concentration of electrolytes to achieve the optimization of comprehensive benefits.

Some additive molecules can interact strongly with $\mathrm{Zn}^{2+}$ ions to adjust the $\mathrm{Zn}^{2+}$ coordination environment; glucose additive was incorporated into the $\mathrm{H}_{2} \mathrm{SO}_{4}$ electrolyte to form a mixed electrolyte [136]. Experiments and theoretical simulations confirmed that $\mathrm{Zn}^{2+}$ ions exhibited a stronger binding interaction with glucose than water molecules (Fig. 17a). Hence, glucose can enter the primary solvation shell of $\mathrm{Zn}^{2+}$ ions, replacing part of the water molecules in the solvent sheath around $\mathrm{Zn}^{2+}$. Thus, $\mathrm{Zn}^{2+}$ mainly existed in the form of glucose- $\mathrm{Zn}^{2+}-5 \mathrm{H}_{2} \mathrm{O}$ solvation structures (Fig. 17b). The significantly decreased electrostatic potential value indicates that the electrostatic repulsion around $\mathrm{Zn}^{2+}$ ions can be relieved, which is beneficial to their rapid migration (Fig. 17c). Likewise, some other additives recently reported, such as glycerol [137], acetonitrile (AN) [138], and ethylene glycol (EG) [139], can also reduce the degree of hydration. Slightly different, some additives can directly interact with water molecules. For example, considering that recrystallization or delamination occurs in the $\mathrm{ZnSO}_{4}$ electrolyte with some liquid alcohol, Hao et al. [140] introduced an antisolvent strategy to the electrolyte. Due to the small molecular volume and high dielectric constant, methanol is added to the $\mathrm{ZnSO}_{4}$ electrolyte as an antisolvent (Fig. 17d). The methanol molecules initially attract free water molecules from the solvation of $\mathrm{Zn}^{2+}$ through hydrogen bonds (Fig. 17e). As the methanol concentration increases, methanol molecules will be inserted into the outer and inner layers of 

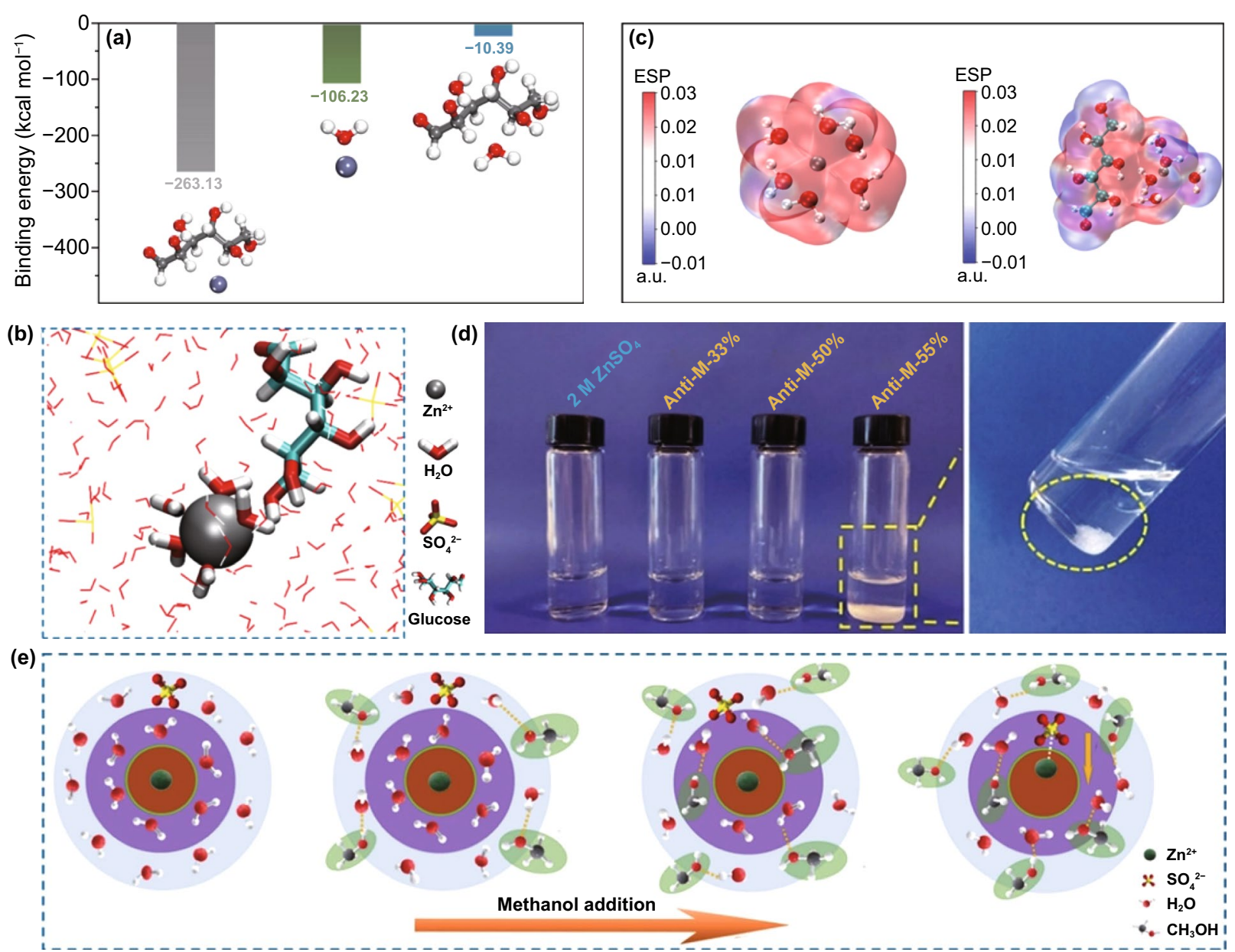

Fig. 17 a Binding energy for $\mathrm{Zn}^{2+}$ with different compounds (glucose and $\mathrm{H}_{2} \mathrm{O}$ ) under DFT calculation; b Partial enlarged 3D snapshot representing $\mathrm{Zn}^{2+}$ solvation structure, obtained from $\mathrm{MD}$ simulations of $\mathrm{ZnSO}_{4}$-glucose system; c Electrostatic potential mapping of the original $\mathrm{Zn}^{2+}-6 \mathrm{H}_{2} \mathrm{O}$ (left) and glucose- $\mathrm{Zn}^{2+}-5 \mathrm{H}_{2} \mathrm{O}$ (right) solvation structures [136]. Copyright 2021, Wiley-VCH. d Preparation of methanol-based antisolvent electrolytes; inset shows recrystallization of $\mathrm{ZnSO}_{4}$ in antisolvent electrolyte of 55\% methanol; e Schematic of changes in the $\mathrm{Zn}^{2+}$ solvent sheath, together with methanol addition [140]. Copyright 2020, Wiley-VCH

the $\mathrm{Zn}^{2+}$ solvation sheath. The exposed $\mathrm{Zn}^{2+}$ ions will recombine with $\mathrm{SO}_{4}{ }^{2-}$ ions, which means that methanol can reduce water activity and disturb the coordination balance between water and $\mathrm{Zn}^{2+}$ ions.

\subsubsection{Suppression of 2D Diffusion}

Additives involving some organic molecules can be adsorbed on the $\mathrm{Zn}$ anode surface, thereby inhibiting the 2D diffusion of $\mathrm{Zn}^{2+}$ ions. Adsorption interactions may be derived from electrostatic induction and chemical bonds between additives and $\mathrm{Zn}$ metal. The strong adsorption interaction is favorable to a stable anode interface. It has been reported that glycerol [137], polyethylene glycol (PEG200) [141], polyethylene oxide (PEO) [142, 143], and other organic molecular additives can achieve adsorption on the anode surface. These additives adsorbed on the metal surface are similar to the artificial non-conductive modified layer. On the one hand, they act as a physical barrier to prevent the surface migration of $\mathrm{Zn}^{2+}$ ions. Therefore, $\mathrm{Zn}^{2+}$ ions form a large number of tiny nuclei at the initial contact site with the metal (Fig. 18a). On the other hand, the groups of some additives can adsorb $\mathrm{Zn}^{2+}$ ions to inhibit surface diffusion, and $\mathrm{Zn}$ preferentially nucleates around the additives fixed on the anode surface (Fig. 18b) [142-144]. 
(a)

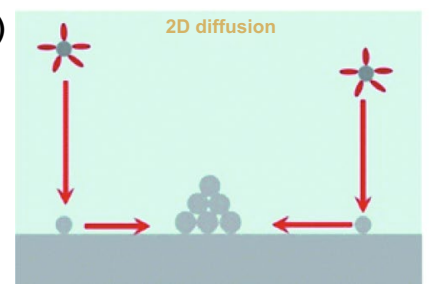

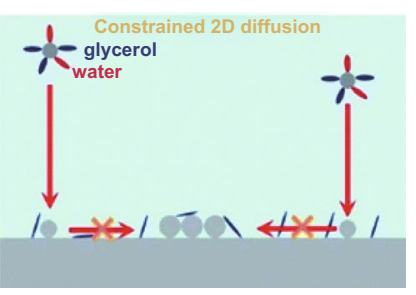

(b)

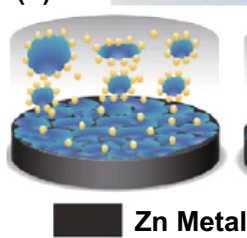

Zn deposition

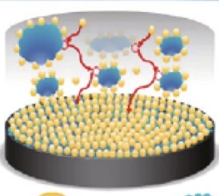

$\mathrm{Zn}^{2+}$

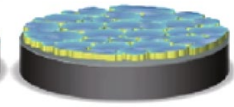

MXene

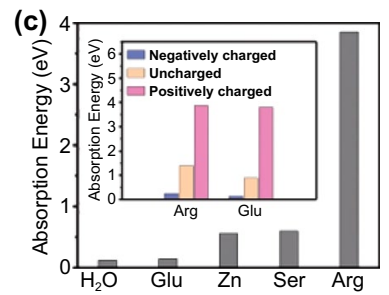

(e)
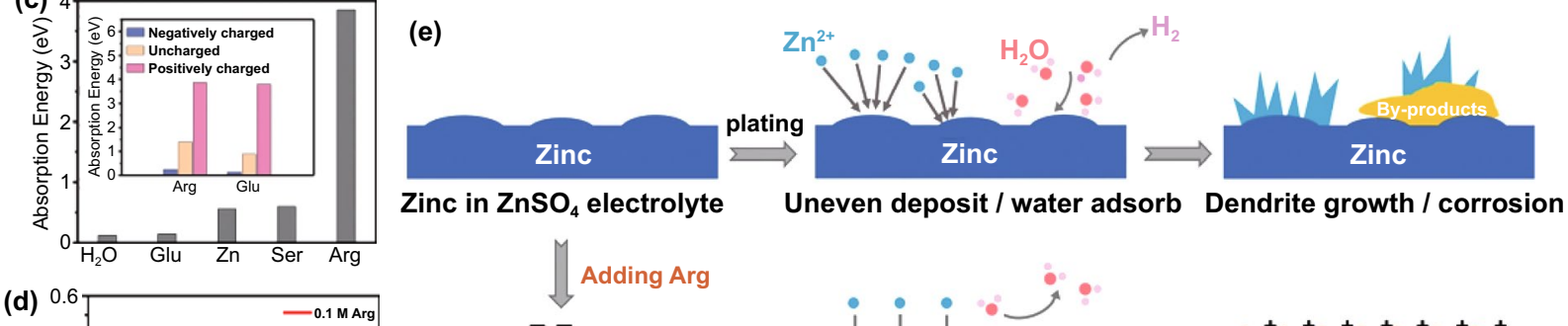
Zinc in $\mathrm{ZnSO}_{4}$ electrolyte

Uneven deposit / water adsorb Dendrite growth / corrosion Adding Arg
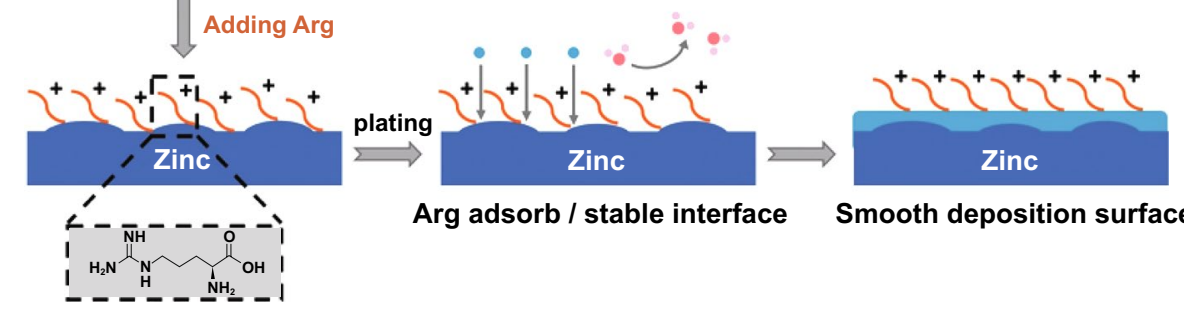

Arg adsorb / stable interface Smooth deposition surface

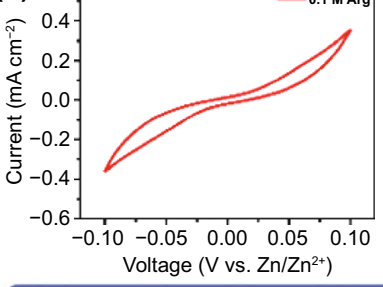

$---\mathrm{NH}_{2}-$
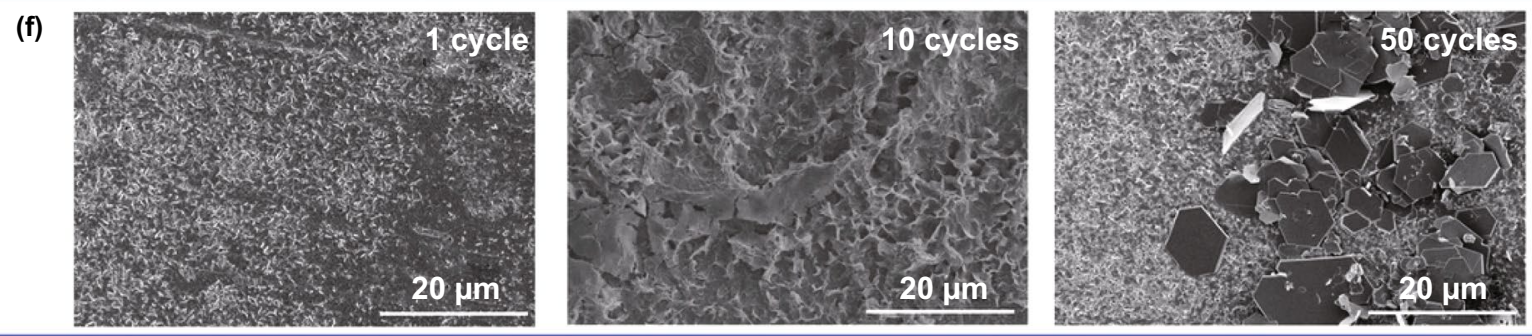

(g)
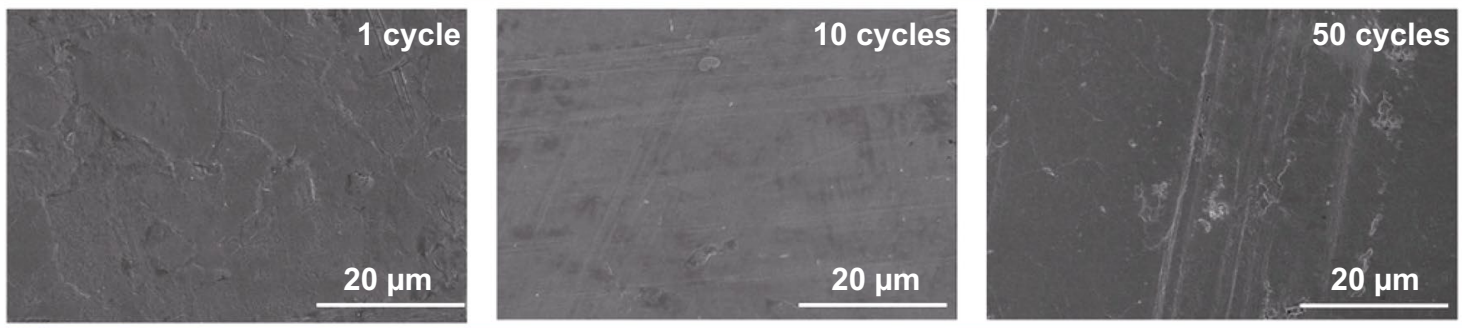

Fig. 18 a Schematics of the $\mathrm{Zn}^{2+}$ diffusion and reduction processes on the bare $\mathrm{Zn}$ electrode in aqueous and hybrid electrolytes, showing that the surface diffusion is constrained in the hybrid electrolyte [137]. Copyright 2021, Royal Society of Chemistry. b Schematic illustration of the effect of MXene additive on the $\mathrm{Zn}$ deposition process [144]. $\mathbf{c}$ Absorption energy of $\mathrm{H}_{2} \mathrm{O}$, Glu, Zn, Ser, and Arg on $\mathrm{Zn}$ surface in mildly acidic electrolyte, respectively. Inset is absorption energy of Arg and Glu with positively charged, uncharged, and negatively charged on $\mathrm{Zn}$ surface, respectively; d CV curve of Zn symmetric cell with $0.1 \mathrm{M}$ Arg solution at $5 \mathrm{mV} \mathrm{s}^{-1}$. e Schematic illustration of $\mathrm{Zn}$ plating behavior with and without Arg additive; surface morphology of $\mathrm{Zn}$ electrode at $1 \mathrm{st}, 10$ th, and 50th cycle $\mathbf{f}$ in bare $\mathrm{ZnSO}_{4}$ electrolyte and $\mathbf{g}$ in $\mathrm{ZnSO}{ }_{4}+\mathrm{Arg}$ electrolyte [145]. Copyright 2021, Wiley-VCH

Resulting from the suppressed 2D diffusion, the subsequent $\mathrm{Zn}$ deposition will grow a dense and smooth $\mathrm{Zn}$ layer. In a recent report of amino acids as additives [145], positively charged amino acids, especially arginine (Arg), will preferentially undergo anodic interface adsorption due to their higher adsorption energy than $\mathrm{H}_{2} \mathrm{O}$ and Zn (Fig. 18c). The adaptive absorption layer of Arg, which belongs to the capacitive adsorption behavior (Fig. 18d), can adapt to the dynamic interface changes and effectively adjust the interface charge during the $\mathrm{Zn}$ electroplating/stripping 
process. According to the chronoamperometry curve, the suppressed $2 \mathrm{D}$ diffusion leads to a uniform $\mathrm{Zn}^{2+}$ ion flux so that the $\mathrm{Zn}^{2+}$ ions in contact with the anode surface tend to react directly to alleviate continuous dendritic growth (Fig. 18e-g).

\subsubsection{Formation of Electrostatic Shielding Layer}

A part of additives can form an electrostatic shielding layer at the tip to inhibit the growth of dendrites (Fig. 19a). During the deposition process, additional cations compete with $\mathrm{Zn}^{2+}$ ions for adsorption, and the one with a lower reduction potential in the additives will be preferentially adsorbed on the initial tip, forming electrostatic shielding, thereby changing the subsequent $\mathrm{Zn}$ deposition behavior [146, 147]. As a proof of concept, tetrabutylammonium sulfate $\left(\mathrm{TBA}_{2} \mathrm{SO}_{4}\right)$ has been used as an electrolyte additive to regulate $\mathrm{Zn}$ deposition (Fig. 19b) [148]. During the plating process, the reduction potential of $\mathrm{TBA}^{+}$cations is lower than that of $\mathrm{Zn}^{2+}$ ions, so the $\mathrm{TBA}^{+}$cations will preferentially adsorb at the tip, which counteracts the tip effect and further drives $\mathrm{Zn}^{2+}$ ions to nucleate around the non-protrusions, thus regulating the deposition behavior of $\mathrm{Zn}^{2+}$ ions. Finally, a smooth deposited layer is formed. In addition to $\mathrm{TBA}_{2} \mathrm{SO}_{4}$, some other metal cation-containing additives also have similar effects, such as $\mathrm{Na}_{2} \mathrm{SO}_{4}$ [149]. Moreover, some stable and polar organic molecules, such as ether [150], can also form an electrostatic shielding layer at the tip (Fig. 19c). In the high electric field around the tip, the polarized molecules are easily attracted to the tip. Good chemical stability ensures that organic molecules will not participate in the transfer of electrons. As a result, the $\mathrm{Zn}$ deposition behavior can also be controlled.
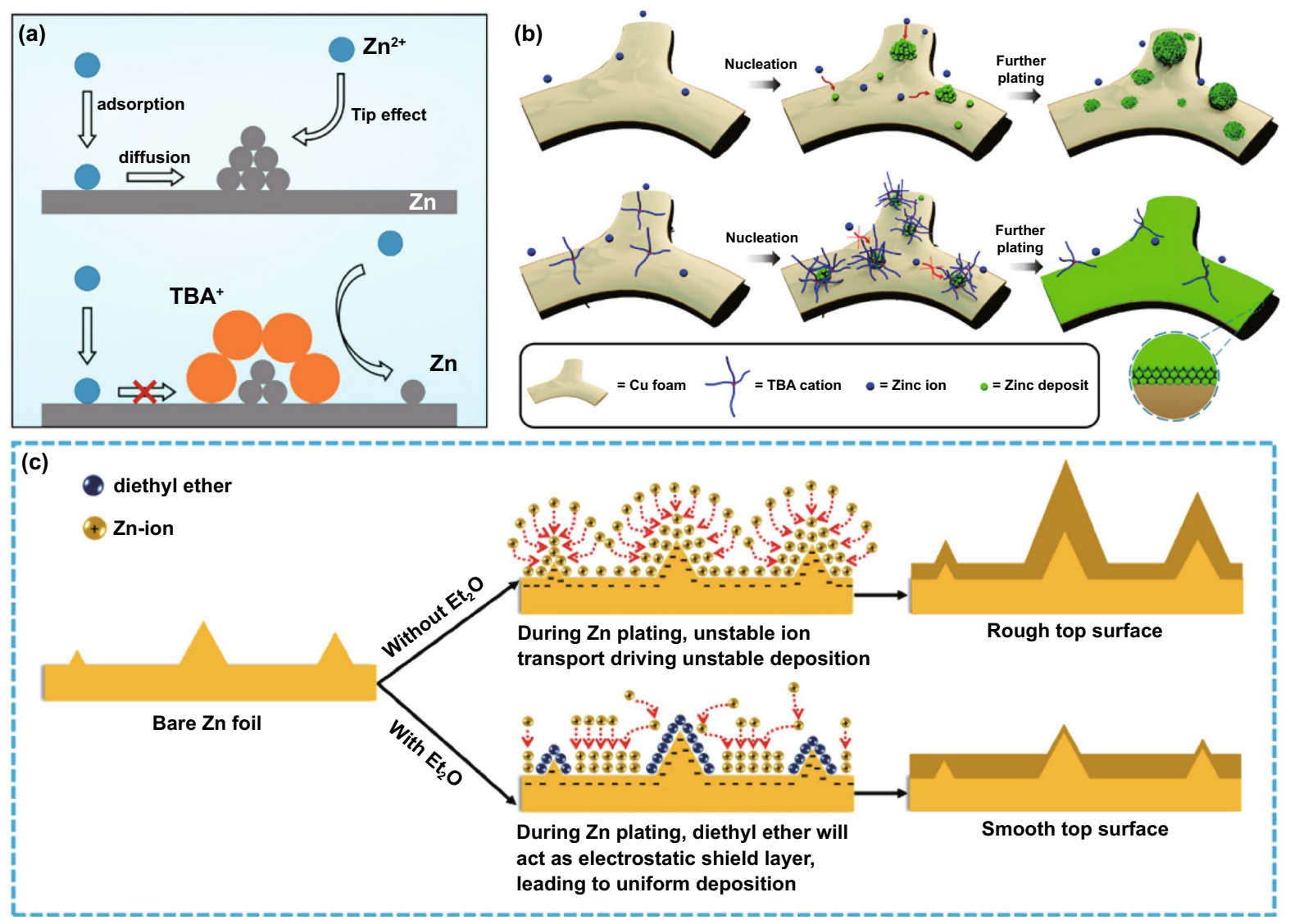

Fig. 19 a Schematics of the $\mathrm{Zn}^{2+}$ ion diffusion and reduction processes on electrodes in $2 \mathrm{M} \mathrm{ZnSO}_{4}$ electrolyte (top) without and (bottom) with $0.05 \mathrm{mM} \mathrm{TBA}_{2} \mathrm{SO}_{4}$; $\mathbf{b}$ Schematic illustrations of $\mathrm{Zn}$ deposition on $\mathrm{Cu}$ foam without or with $\mathrm{TBA}_{2} \mathrm{SO}_{4}$ as an electrolyte additive [148]. Copyright 2020, American Chemical Society. c Schematics of morphology evolution for $\mathrm{Zn}$ anodes in mild aqueous electrolyte with and without $\mathrm{Et}_{2} \mathrm{O}$ additive during $\mathrm{Zn}$ stripping/plating cycling [150]. Copyright 2019, Elsevier 


\subsubsection{Formation of In Situ SEI}

It has been proved that by adding some additives to the applied electrolyte, a dense and uniform SEI layer can be formed in situ on the anode surface to suppress dendrites and side reactions. As we all know, different from the artificial SEI layer, the in situ SEI layer on the anode surface is generally formed by the decomposition of electrolyte components and/or salt anions when electrolyte is in contact with the anode and during the charge/discharge process. Hence, the in situ formation of SEI is related to complex chemical or electrochemical reaction processes. For example, as an additive, a trace amount of $\mathrm{Zn}\left(\mathrm{NO}_{3}\right)_{2}$ is added into the $\mathrm{Zn}(\mathrm{OTF})_{2}$ electrolyte [151]. Before $\mathrm{Zn}$ plating/stripping process, the local $\mathrm{pH}$ increases on the $\mathrm{Zn}$ surface due to the $\mathrm{H}_{2} \mathrm{O}$ reduction in HER. And $\mathrm{Zn}$ reacts easily with $\mathrm{NO}_{3}{ }^{-} / \mathrm{OH}^{-}$to form $\mathrm{Zn}_{5}(\mathrm{OH})_{8}\left(\mathrm{NO}_{3}\right)_{2} \cdot 2 \mathrm{H}_{2} \mathrm{O}$ passivation layer. During the initial $\mathrm{Zn}$ plating/stripping cycles, the typical Zn plating/stripping peaks can be gradually observed in the CV curve of the half-cell (Fig. 20a, b), and the interface impedance approaches that of the additive-free system (Fig. 20c), indicating that there is an activation process. This can be explained by the fact that the passivation layer is converted into $\mathrm{Zn}_{5}\left(\mathrm{CO}_{3}\right)_{2}(\mathrm{OH})_{6}$ layer through metathesis reaction. Then, this layer and salt anions will participate in subsequent reactions. As a result, an electronically insulating and ion-conducting inorganic $\mathrm{ZnF}_{2}-\mathrm{Zn}_{5}\left(\mathrm{CO}_{3}\right)_{2}(\mathrm{OH})_{6}$-organic bilayer SEI is formed on the anode surface (Fig. 20d). The highly flexible organic outer layer can prevent SEI from cracking due to volume changes. The hydrophobic $\mathrm{ZnF}_{2}$ in the inner layer can further enhance desolvation and inhibit $\mathrm{H}_{2} \mathrm{O}$ decomposition and $\mathrm{Zn}$ dendrite growth by preventing direct contact between $\mathrm{Zn}$ and $\mathrm{H}_{2} \mathrm{O}$, but allow $\mathrm{Zn}^{2+}$ ions to transport. Similarly, $\mathrm{ZnF}_{2}$-rich SEI in situ has also been reported in several other works: Introducing trimethylethylammonium trifluoromethanesulfonate $\left(\mathrm{Me}_{3} \mathrm{EtNOTF}\right)$ additive into $\mathrm{Zn}(\mathrm{OTF})_{2}$ electrolyte can form an SEI composed of $\mathrm{ZnF}_{2}, \mathrm{ZnCO}_{3}, \mathrm{ZnSO}_{3}$, and polyanions; introducing $\mathrm{KPF}_{6}$ additive into the $\mathrm{ZnSO}_{4}$ electrolyte can form an SEI composed of $\mathrm{Zn}_{3}\left(\mathrm{PO}_{4}\right)_{2}$ and $\mathrm{ZnF}_{2}$ (ZCS); and introducing $\mathrm{ZnF}_{2}$ additive into $\mathrm{ZnSO}_{4}$ electrolyte can form F-rich SEI. $\mathrm{ZnF}_{2}$ boasting unique merits can increase $\mathrm{Zn}^{2+}$ ion migration and deposition kinetics. At the same time, the high interface energy of SEI component with the $\mathrm{Zn}$ substrate (such as $\left.\mathrm{Zn}_{3}\left(\mathrm{PO}_{4}\right)_{2} / \mathrm{Zn}, \mathrm{ZnF}_{2} / \mathrm{Zn}\right)$ can suppress dendrite growth by promoting lateral rather than vertical $\mathrm{Zn}^{2+}$ migration and deposition [152-154]. Additionally, it can be seen that the change of electrolyte $\mathrm{pH}$ generally accompanies the SEI formation process involving $\mathrm{H}_{2} \mathrm{O}$ decomposition. In addition to the above mentioned, the introduction of $\mathrm{Zn}\left(\mathrm{H}_{2} \mathrm{PO}_{4}\right)_{2}$ salt into the $\mathrm{Zn}\left(\mathrm{CF}_{3} \mathrm{SO}_{3}\right)_{2}$ electrolyte can form a hopeite $\left(\mathrm{Zn}_{3}\left(\mathrm{PO}_{4}\right)_{2} \cdot 4 \mathrm{H}_{2} \mathrm{O}\right)$ layer in situ (Fig. 20e) [155]. The formation of this dense SEI is entirely dependent on HER, which can change the local $\mathrm{pH}$. The increased local concentration of $\mathrm{OH}^{-}$anions creates conditions for the spontaneous formation of SEI in situ. This process is to transform the shortcomings caused by HER into advantages. Both this SEI and its formation process can suppress side reactions. In addition, it has recently been reported that in the $\mathrm{ZnSO}_{4}$ electrolyte, the introduction of $\mathrm{LiCl}$ additives will preferentially form a $\mathrm{Li}_{2} \mathrm{O} / \mathrm{Li}_{2} \mathrm{CO}_{3}$ layer [44]; introducing $\mathrm{SnCl}_{2}$ additive into $\mathrm{ZnCl}_{2}$ electrolyte can form $\mathrm{Sn} / \mathrm{Zn}_{5}(\mathrm{OH})_{8} \mathrm{Cl}_{2} \cdot \mathrm{H}_{2} \mathrm{O}$ double layer in situ [156]. Based on the above analysis, it can be concluded that in situ SEI is mainly through chemical reaction and mechanical inhibition to alleviate dendrite growth and side reactions. Accordingly, electronic insulation, ion conduction, high Zn/SEI interface energy, and large bulk modulus are essential for a stable in situ SEI. Importantly, the formation process of SEI is similar to the negative feedback effect. The continuous decomposition of electrolyte components and/or salt anions can promote the gradual completion of SEI, which in turn inhibits this process, thereby preventing unrestricted SEI growth. As long as the conditions are sufficient, this process will proceed spontaneously. Almost all reported in situ SEIs have a self-repair function, which is not available in artificial SEIs. Therefore, the strategy of constructing in situ SEI is worthy of in-depth study to realize stable and reversible anodes.

\subsection{Non-Liquid Electrolyte}

Different from liquid electrolytes, non-liquid electrolytes with unique properties have been designed. Generally, with a decrease in free water content, non-liquid electrolytes have lower ionic conductivity. Fortunately, the controllability of non-liquid components can make up for the shortcomings and achieve performance close to or even better than that of liquid electrolytes. Therefore, a stable and reversible anode interface can be realized, and the high designability of non-liquid electrolytes significantly broadens the working 

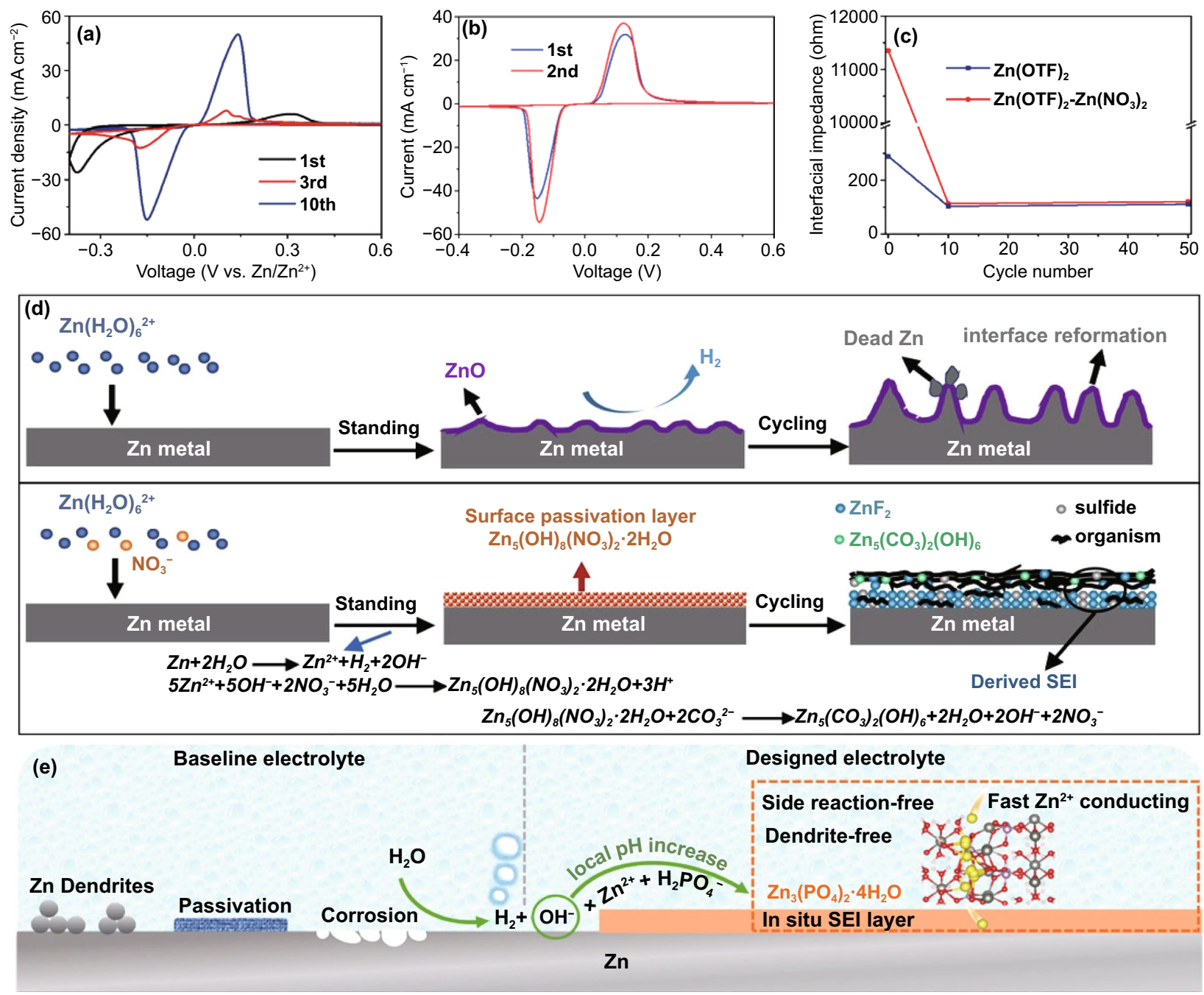

Fig. $20 \mathrm{CV}$ curves of $\mathrm{Zn} \| \mathrm{Ti}$ half cells a with and $\mathbf{b}$ without $\mathrm{Zn}\left(\mathrm{NO}_{3}\right)_{2}$ additive; $\mathbf{c}$ Interfacial impedance measured from $\mathrm{Zn} \| \mathrm{Zn}$ cells in $\mathrm{Zn}(\mathrm{OTF})_{2}$ electrolytes with and without $\mathrm{Zn}\left(\mathrm{NO}_{3}\right)_{2}$ additive under cycling; $\mathbf{d}$ Illustration of surface evolution mechanism in $\mathrm{Zn}(\mathrm{OTF})_{2}$ electrolytes with and without $\mathrm{Zn}\left(\mathrm{NO}_{3}\right)_{2}$ additive. [151]. Copyright 2021, Wiley-VCH. e Schematic illustration of the $\mathrm{Zn}$ surface evolution and characterization of Zn electrodes in the baseline and designed electrolytes [155]. Copyright 2021, Wiley-VCH

environment range of aqueous ZIBs. This section mainly analyzes solid-state electrolytes, hydrogel electrolytes, and other non-liquid electrolytes.

\subsubsection{Solid-state Electrolytes}

Due to excellent chemical stability, solid-state electrolytes (SSEs) exhibit high safety. The ion guidance derived from the mechanical structure and surface chemistry of the electrolyte can restrain the $\mathrm{Zn}$ dendrites to a certain extent, and the elimination of water can effectively solve the anode interface issues caused by side reactions. However, it is also obvious that the low ion migration rate and high boundary impedance are tricky challenges for all-solid-state electrolytes. Therefore, there are few real all-solid-state electrolytes [20], and most reported solid electrolytes involve a certain amount of liquid plasticizer, such as poly(vinylidene fluoride-hexafluoropropylene)/ poly(ethylene oxide) film filled with ionic-liquid-based $\mathrm{Zn}$ salt and water-soaked $\mathrm{Zn}$ perfluorosulfonate membrane (ZPSAM) [157, 158]. Impressively, the single ion $\mathrm{Zn}^{2+}$ SSEs based on the post-synthesis modified MOF-808 have a fixed anion microporous host (Fig. 21a) [159]. At higher humidity, these microporous hosts will absorb water and solvate the $\mathrm{Zn}^{2+}$ ions in the pores. Conductive and highconcentration $\mathrm{Zn}\left(\mathrm{H}_{2} \mathrm{O}\right)_{6}{ }^{2+}$ can accelerate ion transfer. Since 

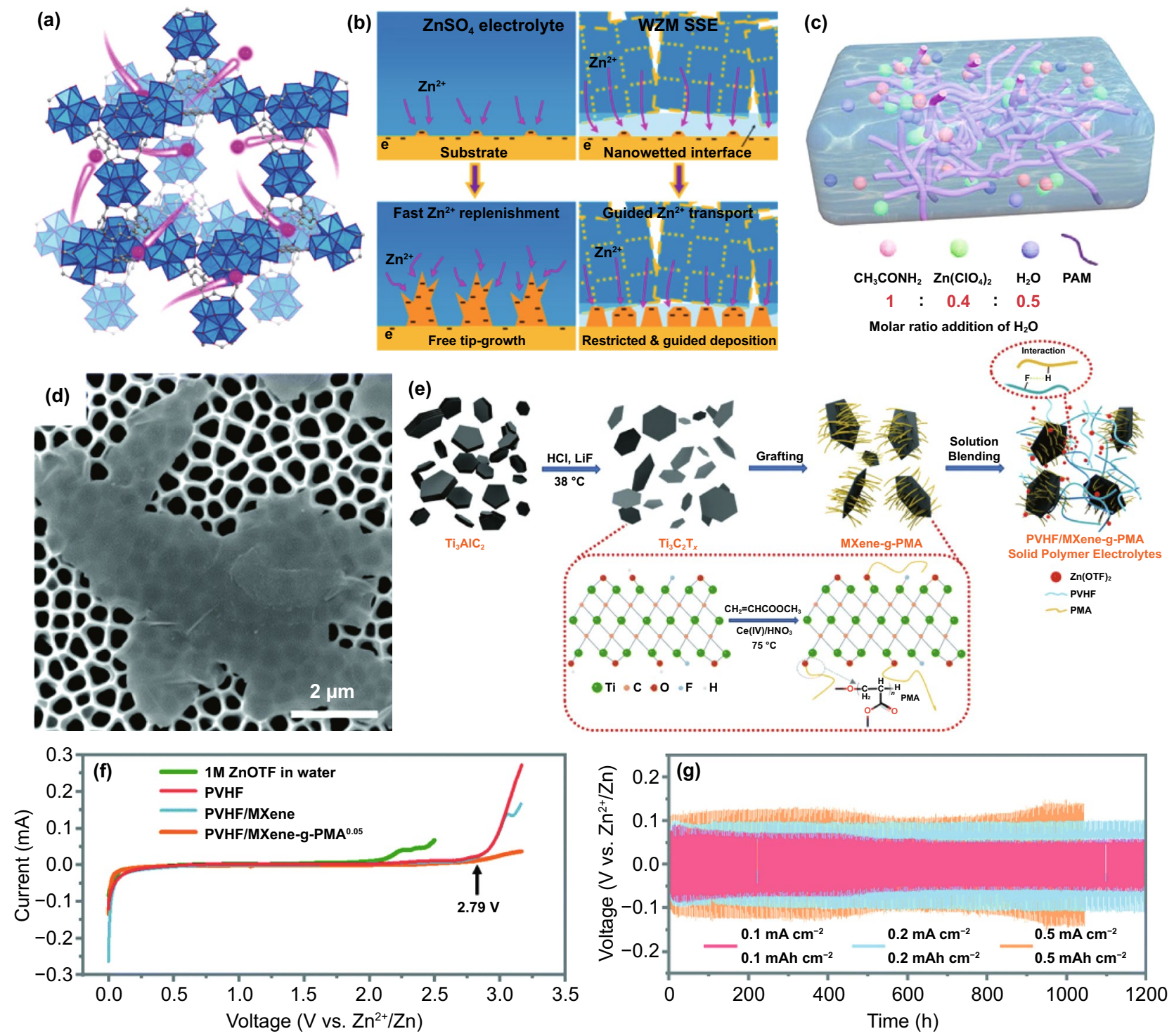

Fig. 21 a Crystal structure of ZnMOF-808. Blue polyhedrons represent $\mathrm{Zr}-\mathrm{O}$ clusters, and $\mathrm{Zn}^{2+}$ ions are highlighted by pink balls; b Proposed mechanism for the different deposition behaviors of $\mathrm{ZnSO}_{4}$ aqueous electrolyte (left) and WZM SSE (right) [159]. Copyright 2018, Elsevier. c Schematic diagram of IL-PAM composition and structure [161]. Copyright 2021, American Chemical Society. d SEM image of MXene-g-PMA; e Schematic illustration of the overall preparation process of the SPEs; $\mathbf{f}$ LSV of the SPEs (scan rate $0.5 \mathrm{mV} \mathrm{s}^{-1}$ ); $\mathbf{g}$ Galvanostatic Zn plating/ stripping in the Zn/Zn symmetrical cells based on different current densities with different plating capacities [162]. Copyright 2021, Royal Society of Chemistry

the water released by desolvation wets the reaction interface and the sub-nano-tunnel guides the deposition site, the $\mathrm{Zn}$ deposition morphology is more uniform, dense, and smooth (Fig. 21b).

Due to combining the advantages of the high ionic conductivity of the inorganic electrolyte and the high interface compatibility between the polymer electrolyte and the metal anode, solid polymer electrolytes (SPEs) have attracted significant attention, and they are the most reported SSEs in ZIBs [52, 160]. Recently, based on acetamide/zinc perchlorate hexahydrate ionic liquid, polyacrylamide polymer electrolytes (IL-PAM) have been synthesized (Fig. 21c) [161]. Similar to the electronic insulation artificial modification layer, cross-linked PAM as a 3D skeleton can suppress dendrites. The wettability of water increases the ionic conductivity of IL-PAM. Since water is a trace amount, there 
is almost no free water in the electrolyte, corresponding to inhibited HER.

Compositing fillers into the polymer matrix can enhance the performance of SPEs. In particular, fillers with rich surface chemistry and a large surface area can significantly increase the ionic conductivity of SPEs. This is mainly achieved by promoting the dissociation of $\mathrm{Zn}$ salts, forming ion migration pathways on the filler surface, and acting as plasticizers to reduce polymerization crystallinity and enhance chain migration [162]. Based on this strategy, SPEs containing suitable fillers can show excellent performance without a liquid phase. Thus, the all-solid-state electrolyte has been proposed. For example, the 2D material MXene was chemically grafted with poly(methyl acrylate) to form a composite material (denoted as MXene-g-PMA) (Fig. 21d). As a filler, it was dispersed into poly(vinylidene fluorideco-hexafluoropropylene) (PVHF) (Fig. 21e). Compared with PVHF and PVHF/MXene, PVHF/MXene-g-PMA had higher ion conductivity. Compared with common aqueous electrolytes, these SPEs can obtain an electrochemical stability window as high as $2.79 \mathrm{~V}$ (relative to $\mathrm{Zn}^{2+} / \mathrm{Zn}$ ) (Fig. 21f). The SPE-based symmetric battery cycled stably for more than $1000 \mathrm{~h}$ without the formation of dendrites at room temperature (Fig. 21g) [162].

\subsubsection{Hydrogel Electrolyte}

With the application of flexible wearable devices, batteries with hydrogel electrolytes have attracted much attention due to their high electrochemical performance and good mechanical properties. The hydrogel network composed of abundant polymer chains through physical/chemical crosslinking macroscopically displays the shape and volume of the quasi-solid [163]. However, since the hydrophilic group of the polymer chain can absorb a large amount of water, the hydrogel electrolyte exhibits a conductivity close to that of a liquid. Meanwhile, water molecules are restricted instead of freely spreading, indicating that HER can be alleviated to a certain extent [164]. The fixed polymer chains play a vital role in ion transfer and $\mathrm{Zn}$ deposition. For example, polyanionic hydrogel electrolyte poly-2-acrylamide-2-methyl-1-propanesulfonate zinc (PAMPSZn) was synthesized through ion exchange and free radical polymerization (Fig. 22a) [165]. The fixed polyanionic chain containing
$-\mathrm{SO}_{3}{ }^{2-}$ anions guided and accelerated ion transfer to reduce concentration polarization; PAMPSZn hydrogel electrolyte had high ionic conductivity of $15.6 \mathrm{mS} \mathrm{cm}^{-1}$. And the limited contact between $-\mathrm{SO}_{3}{ }^{2-}$ and $\mathrm{Zn}$ metal surface suppressed side reactions. Moreover, the network structure restricted by polyanionic chains served as $\mathrm{Zn}^{2+}$ ion transfer channels to facilitate uniform $\mathrm{Zn}$ deposition (Fig. 22b). Compared with the $\mathrm{ZnSO}_{4}$ aqueous electrolyte, the $\mathrm{Zn}$ anode in the PAMPSZn gel electrolyte exhibited a more uniform surface morphology during the plating/stripping process. In addition, $\mathrm{Zn}$ alginate (ALG-Zn) [166], gelatin-based SSE (GSE) [167], borax cross-linked polyvinyl alcohol/glycerin (PVA-B-G) [168], etc., have similar functions as hydrogel electrolytes.

Flexible energy storage devices usually face many practical challenges such as extremely low temperature, bending, compression, twisted, or even puncturing. Extraordinarily, superior to liquid or solid electrolytes, hydrogel electrolytes exhibit many practical advantages to solve these problems. All-round hydrogel electrolyte (denoted as CT3G30) has been reported, which employs cotton as the raw material of polymetric framework and tetraethyl orthosilicate (TEOS) as the cross-linker (Fig. 22c, d) [169]. A large number of metal salts $\left(\mathrm{ZnSO}_{4}\right.$ and $\mathrm{MnSO}_{4}$ ) in CT3G30 can lower the freezing point of the aqueous system. Meanwhile, the strong interaction between the hydroxyl groups of the cellulose framework and water molecules can suppress ice crystallization of water molecules through hydrogen bonds. Based on these synergies, CT3G30 exhibits a strong antifreezing ability, implying that this hydrogel electrolyte can be adapted to the operating environment at extremely low temperatures (Fig. 22e, f). Furthermore, CT3G30 possesses very high tensile strength, which derives from proper crosslinking inside the hydrogel. Benefit from a large number of hydrogen bonds that are easy to break and reform as well as $\mathrm{Si-O}-\mathrm{Si}$ bonds with excellent chain mobility and high reversibility, CT3G30 shows good self-healing ability (Fig. 22g). Also, the high abundance of hydrogen bonds ensures strong adhesion properties. Based on the above characteristics, the practical applicability of CT3G30 hydrogel electrolyte will be greatly enhanced. Similarly, there are many reports of hydrogel electrolytes with unique properties, such as $\mathrm{PVA} / \mathrm{Zn}\left(\mathrm{CH}_{3} \mathrm{COO}\right)_{2} /$ $\mathrm{Mn}\left(\mathrm{CH}_{3} \mathrm{COO}\right)_{2}(\mathrm{PVA}-\mathrm{Zn} / \mathrm{Mn})[170]$ and $\mathrm{Zn}\left(\mathrm{CF}_{3} \mathrm{SO}_{3}\right)_{2}$ polyacrylamide (PAM) [171]. 


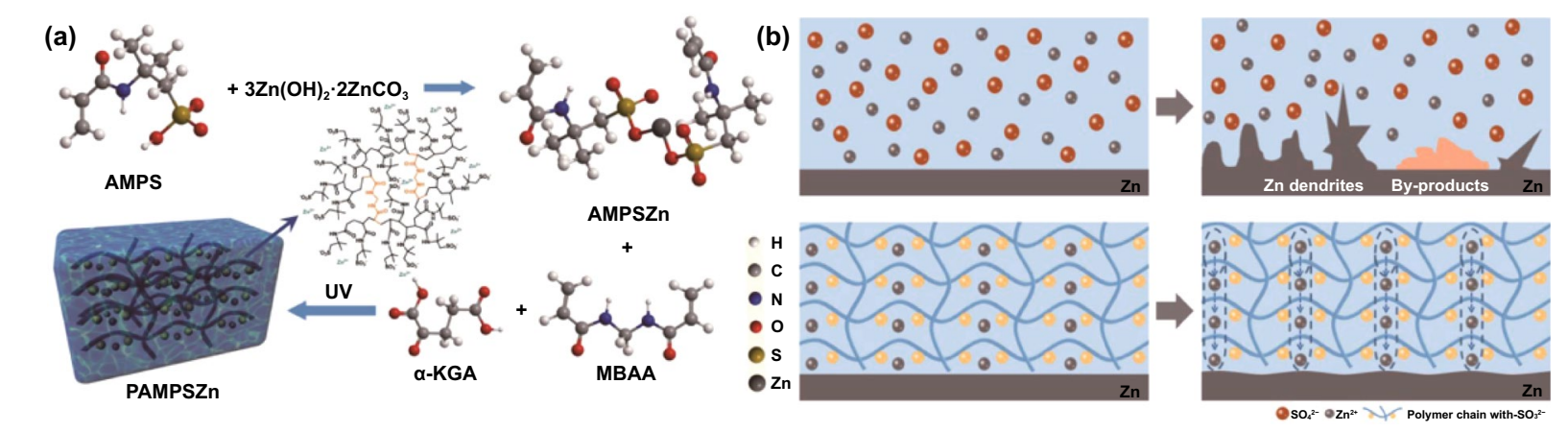

(c)

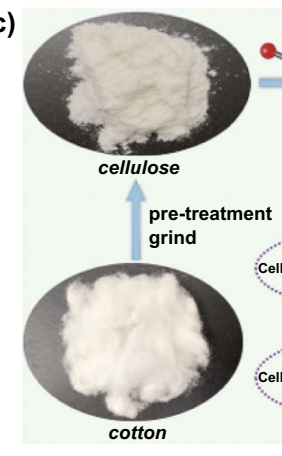

(e)

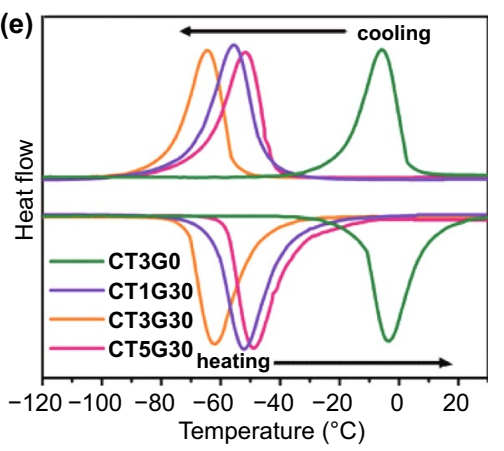

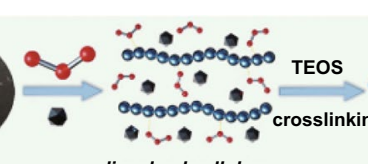

dissolved cellulose

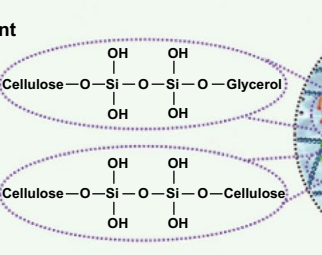

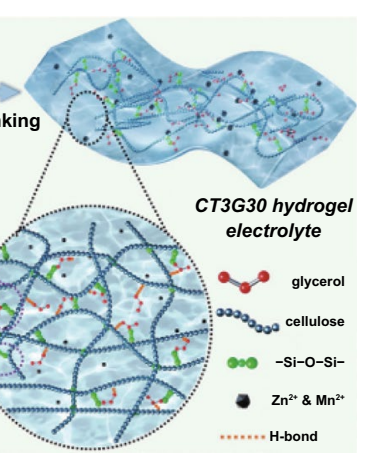
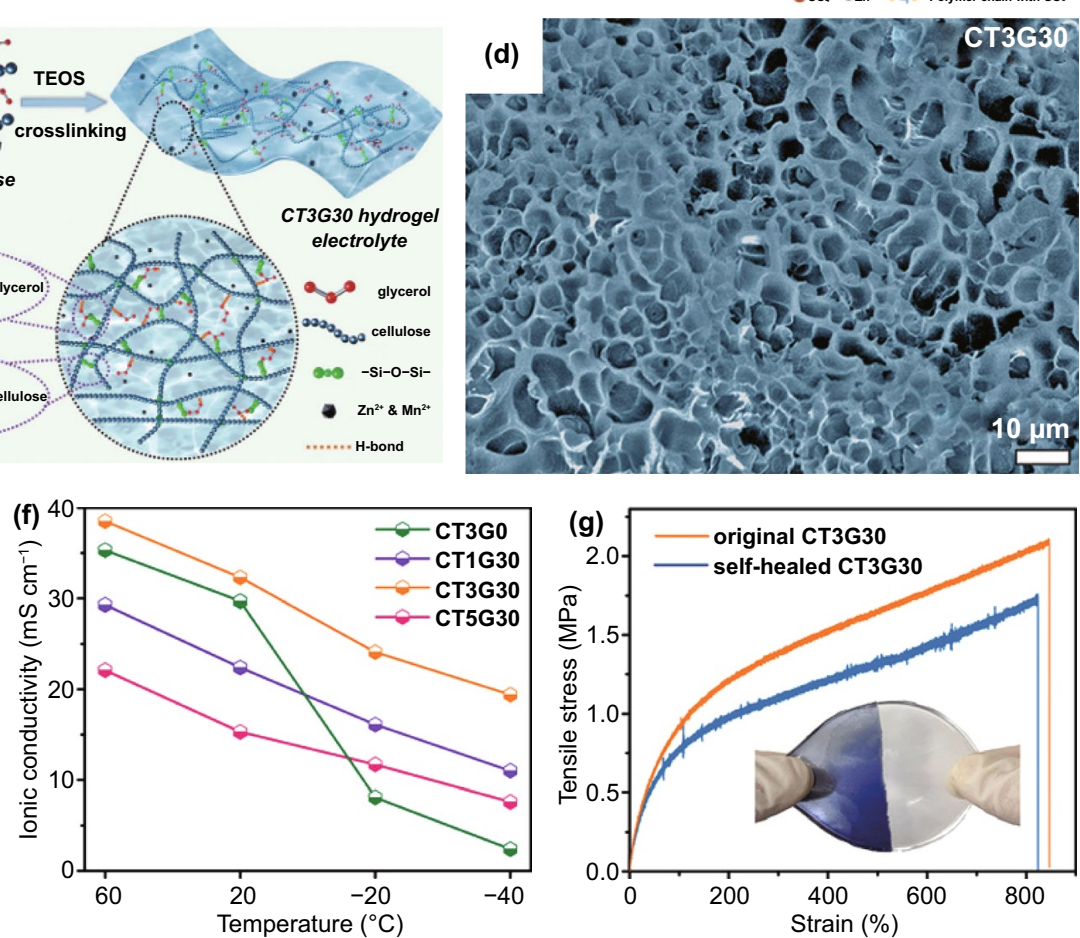

Fig. 22 a Schematic synthesis of PAMPSZn hydrogel electrolyte; b The mechanism of $\mathrm{Zn}$ deposition/stripping with $\mathrm{ZnSO}_{4}$ aqueous electrolyte and PAMPSZn hydrogel electrolyte [165]. Copyright 2020, Elsevier. c Synthesis schematic of the CT3G30 hydrogel electrolyte. d SEM image of freeze-dried CT3G30; e DSC curves and $\mathbf{f}$ ionic conductivity values of CT3G30; $\mathbf{g}$ Tensile $\sigma-\varepsilon$ curves of the original CT3G30 and self-healed CT3G30 [169]. Copyright 2020, Wiley-VCH

\subsubsection{Other Non-liquid Electrolyte}

In addition to hydrogels and solid electrolytes, other materials have also been explored for non-liquid electrolytes. A novel inorganic highly concentrated colloidal electrolyte (HCCE) has been proposed, which is induced by palygorskite nano-inorganic materials [172] (Fig. 23a). The $\mathrm{Zn}^{2+}$ ion in the solvent can strongly interact with palygorskite and insert into its inner crystal through an isomorphic substitution reaction. Since this is a reversible process, the metal cations $\left(\mathrm{Zn}^{2+}\right.$ and $\left.\mathrm{Mg}^{2+}\right)$ in the solvent and palygorskite will continuously and spontaneously exchange, thereby achieving a dynamic balance of the $\mathrm{Zn}^{2+}$ ion concentration. Driven by the concentration gradient in the electrolyte, $\mathrm{Zn}^{2+}$ ions eventually migrate between the anode and the cathode (Fig. 23b). On the one hand, the hydroxyl group in palygorskite fixes water molecules by forming hydrogen bonds; on the other hand, the ion exchange process destroys the water solvation sheath of metal cations, both of which are beneficial to reduce the desolvation energy barrier and inhibit side reactions at the anode interface. In addition, by forming an ion-conducting protective layer on the anode surface, HCCE can further suppress dendrites and side reactions (Fig. 23c). As a result, when the anode 


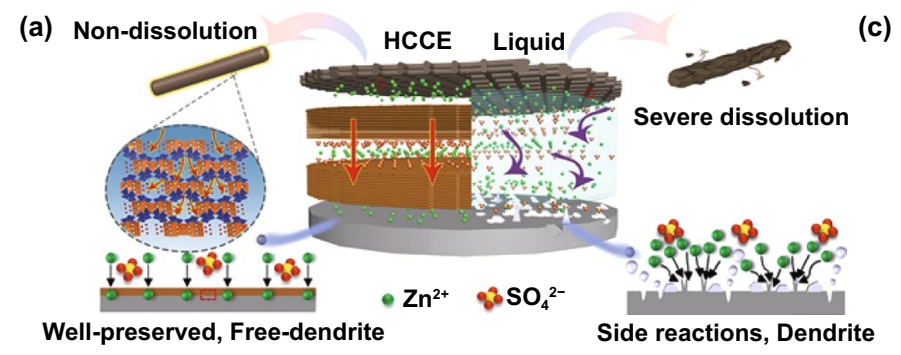

(c) HCCE

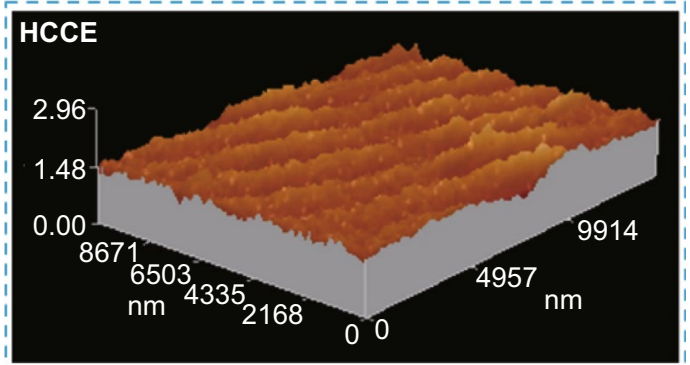

(b)

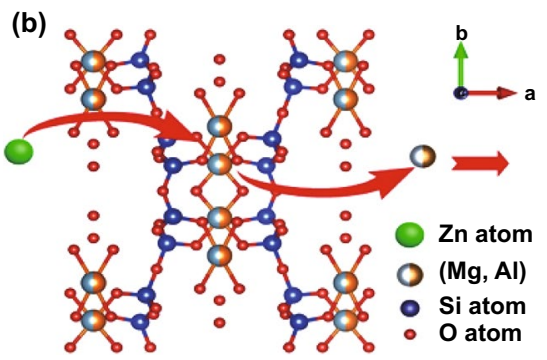

Raw material
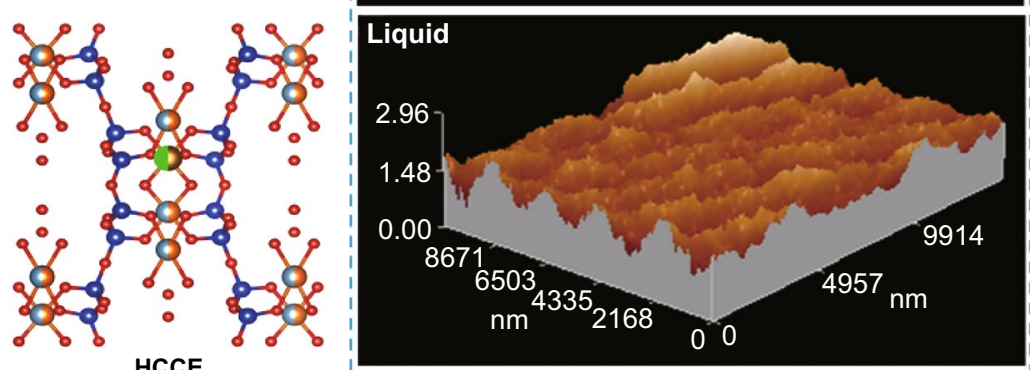

(d)

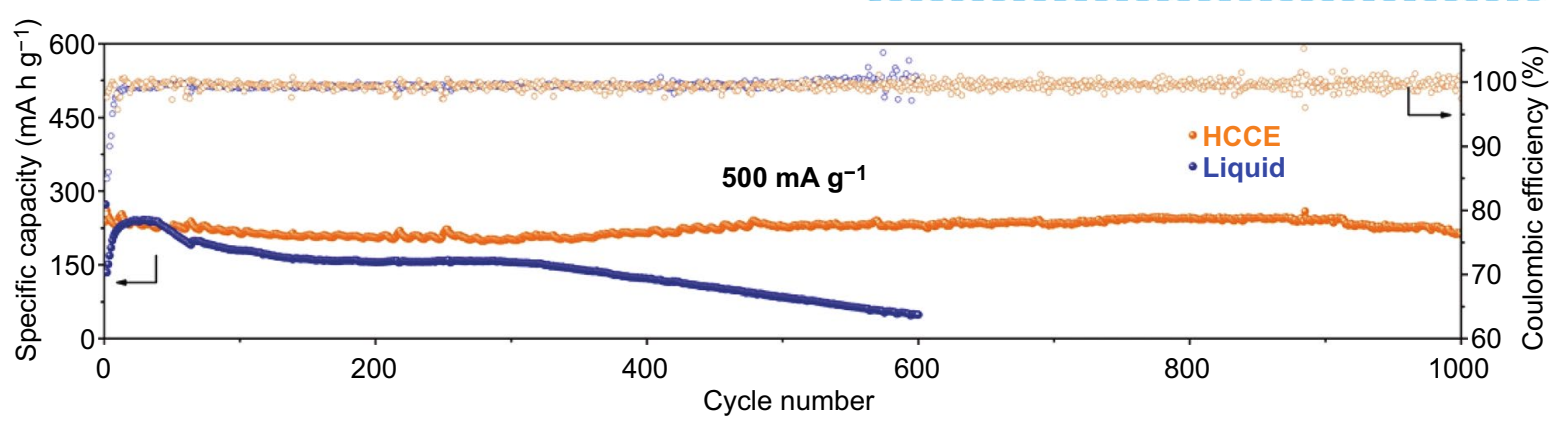

Fig. 23 a Schematic diagram of interface protection effect in HCCE and liquid electrolyte; b Ion exchange diagrams in the HCCE; c AFM images of (top) anode of the battery with HCCE and (bottom) the battery with liquid electrolyte cycled for 200 cycles $1000 \mathrm{~mA} \mathrm{~g}^{-1}$; d Long-life cycling performance of the cell with HCCE and liquid electrolyte at $500 \mathrm{~mA} \mathrm{~g}^{-1}$ [172]

was $\mathrm{Zn}$ and the cathode was $\mathrm{MnO}_{2}$, the battery containing HCCE maintained almost $100 \%$ capacity after 1000 cycles at $500 \mathrm{~mA} \mathrm{~g}^{-1}$ (Fig. 23d).

\subsection{Separator Design}

As a crucial part of the battery configuration, the separator regulates the migration of ions in the electrolyte and isolates the two electrodes to prevent direct contact. Thus, the separator also has a significant impact on the performance of the anode. At the same time, considering that dendrite growth may pierce the separator and cause short circuits accordingly, it is also an effective strategy to design a reliable multifunctional separator. At present, although glass fiber (GF) separators are extensively used in aqueous ZIBs in the laboratory, due to the high cost, low ionic conductivity, and terrible mechanical strength, they are still difficult to meet the upgrade requirements of battery performance. Therefore, there is an increasing desire for a novel multi-function separator. Since it is related to all parts of the battery rather than a single mental anode, the research on the separator is of great significance, and it may be the next research hot spot for aqueous batteries.

Obviously, like the GF separator (Fig. 24a), the traditional porous separators mainly mechanically adjust the mass flux in the solution, which mainly depends on their physical properties, such as mechanical properties, structural morphology, and wettability of the electrolyte. Excellent physical properties of separators play an important role in increasing ion conductivity, homogenizing concentration field, and electric field. Continuing this modification strategy, a $\mathrm{Zn}^{2+}$-substituted Nafion separator (Zn-Nafion) was designed (Fig. 24b) [173]. Compared with the GF separator, the Zn-Nafion separator has dominant physical 

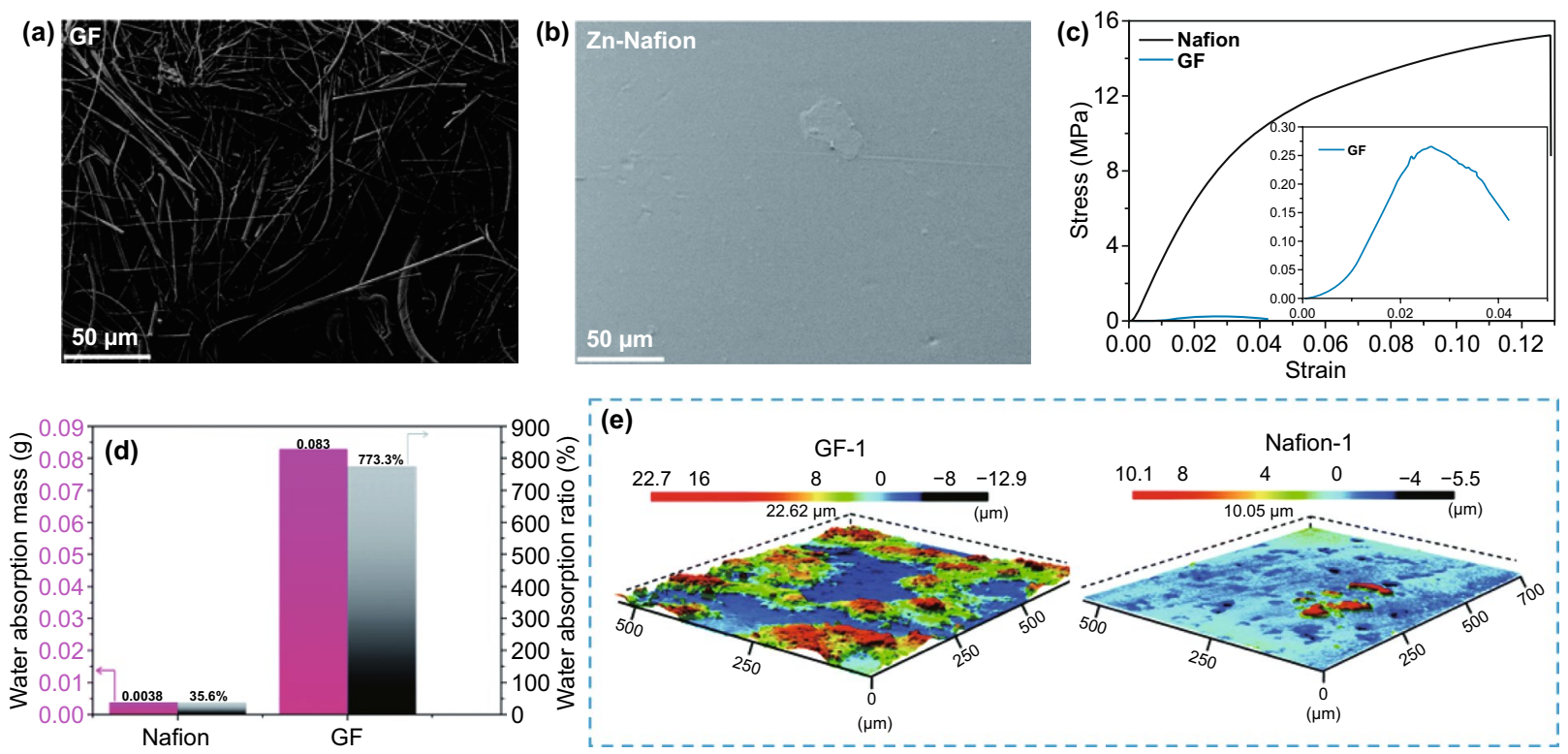

Fig. 24 SEM images of a GF and $\mathbf{b}$ Zn-Nafion separators; $\mathbf{c}$ The stress-strain curves (the inset is a magnified stress-strain curve of the GF separator); $\mathbf{d}$ The water uptake; e The 3D height images for the surface of the (right) GF separator and (left) Zn-Nafion separator after the first cycle [173]. Copyright 2021, Royal Society of Chemistry

properties: High mechanical strength enables better tolerance to dendrites (Fig. 24c), and low water absorption can reduce electrolyte consumption corresponding to less dissolved oxygen (Fig. 24d). At the same time, the thin structure is beneficial to accelerate the transfer of $\mathrm{Zn}^{2+}$ and increase the energy density of the battery. Based on these merits, Zn-Nafion separator promoted the uniform $\mathrm{Zn}$ deposition and reduced the concentration polarization (Fig. 24e). Note that the cost of the Zn-Nafion separator is $47.3 \%$ lower than that of the glass fiber separator, which means that the commercialization of the former is more promising.

In addition to mechanically regulating the $\mathrm{Zn}^{2+}$ ion transfer, designing a separator that can interact with ions can further enhance the anode performance. This can be achieved by attaching other attributes to the separator, such as zincophilicity, electronic conductivity, directional polarization electric field, and low lattice mismatch. Correspondingly, a stable Janus separator facing $\mathrm{Zn}$ anode is reported (Fig. 25a) [174]. Vertical graphene (VG) carpet was directly grown in situ on one side of the commercial glass fiber separator through plasma-enhanced chemical vapor deposition (PECVD) technology. The 3D VG conductive network with a high surface area can uniform electric field distribution and reduce local current density. By introducing oxygen and nitrogen dopants to improve Zn affinity, the Janus separator can effectively and selectively regulate the $\mathrm{Zn}^{2+}$ ions flux. Similarly, compounding MOF/rGO on polypropylene/polyethylene (PP/PE) substrate can obtain a dual-function separator (Fig. 25b) [175]. On the one hand, MOF regulates the uniform $\mathrm{Zn}^{2+}$ ion flux with the assistance of its sub-nanometer anion channel. On the other hand, rGO acts as a conductive layer to reduce the potential microscopic difference on the $\mathrm{Zn}$ surface, which can enhance the corrosion resistance of the anode. Slightly different, compared to the conductive network, a zincophilic metal layer constructed on the separator facing the anode side, such as metal Sn-modified layer [176], can change the location of $\mathrm{Zn}$ deposition (Fig. 25c). Since the anode and the separator modification layer are both electronically conductive and connected, an equipotential surface can be formed between them, implying a uniform electric field between the anode and the separator. The location of $\mathrm{Zn}$ deposition is no longer limited to the anode surface. Instead, there is also $\mathrm{Zn}$ deposition on the separator surface. The restricted $\mathrm{Zn}$ growth direction can eliminate the possibility of dendrites piercing the separator. It can be known that the electronically conductive separator facing the anode side can be regarded as a 3D continuation of the $\mathrm{Zn}$ anode, allowing the space to expand to guide $\mathrm{Zn}$ deposition. In addition, with the help of the Maxwell-Wagner 


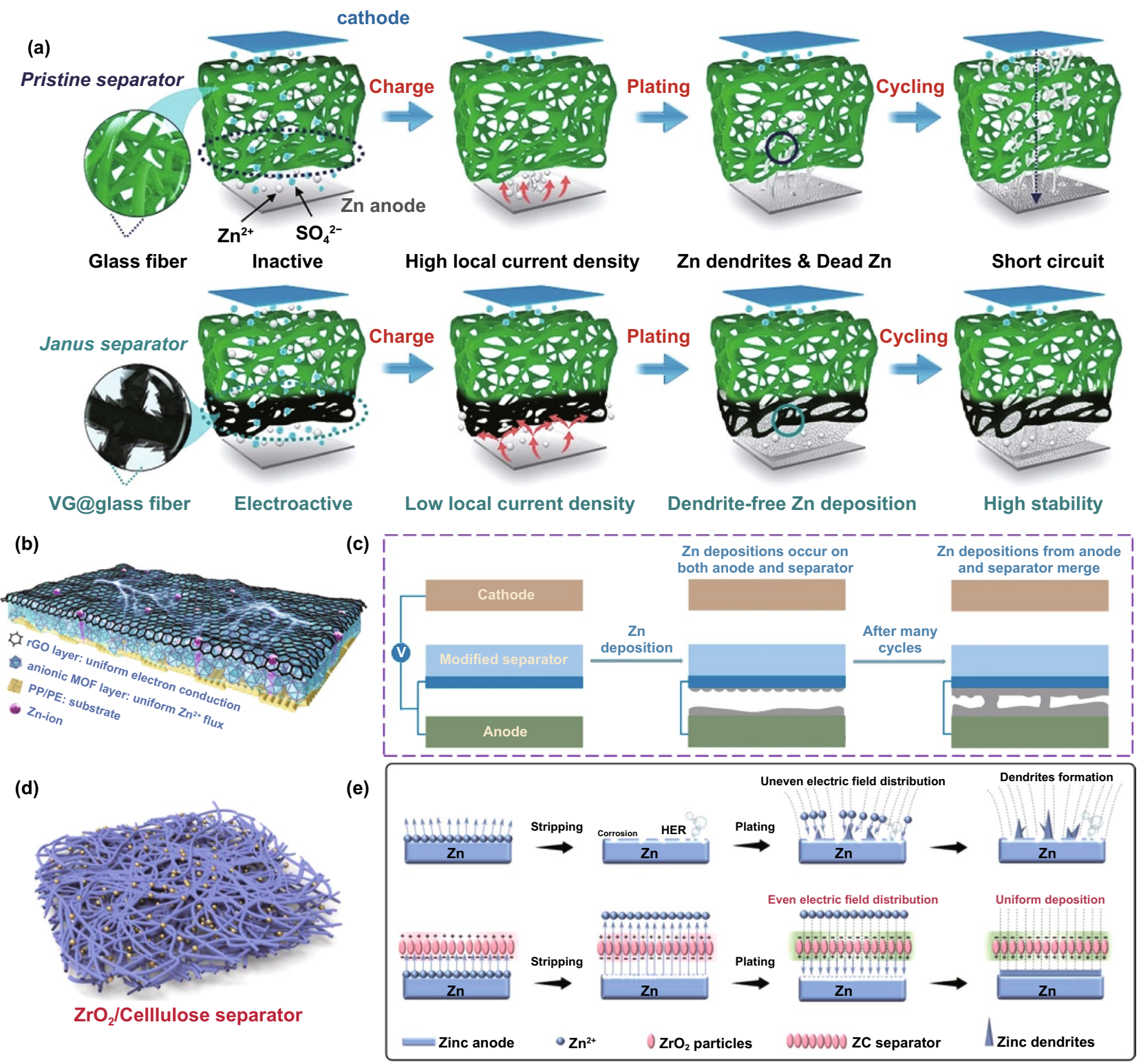

Fig. 25 a Schematics illustrating (top) a pristine glass fiber separator and (bottom) a Janus separator targeting stabilized $\mathrm{Zn}$ anode [174]. Copyright 2020, Wiley-VCH. b Schematic illustration for the Janus separator [175]. c Schematic illustration of Zn deposition in the contact region of the modified separator and the anode [176]. d Schematic illustration for the ZC separator; e Schematic illustration of possible migration process of $\mathrm{Zn}^{2+}$ when passing through the cellulose and ZC separators [177]. Copyright 2021, Elsevier

polarization effect of $\mathrm{ZrO}_{2}$, the composite separator made of cellulose nanofibers and ceramic particles can generate a directional polarization electric field, which can guide uniform $\mathrm{Zn}$ deposition, increase ion conductivity, and inhibit anion transfer (Fig. 25d, e), thereby stabilizing the $\mathrm{Zn}$ anode and suppressing side reactions [177]. As mentioned earlier, low lattice matching can induce the crystal orientation of $\mathrm{Zn}$ deposition. Due to the perfect lattice matching between $(002)_{\mathrm{Zn}}$ and $(002)_{\mathrm{GO}}$, the cellulose/GO composite separator (CG) can also promote the preferential orientation of $\mathrm{Zn}$ crystals along the horizontal direction, resulting in nondendritic Zn electrodeposition [178]. 


\subsection{Other Strategies}

Apart from the representative strategies introduced above, some other meaningful attempts have also been continuously made to solve the issue at the anode interface, such as regulating charge/discharge protocols, changing the battery configuration.

The various strategies currently proposed to inhibit the growth of dendrites are based on prevention, but these strategies will fail once $\mathrm{Zn}$ dendrites are formed. Therefore, a strategy to actively eliminate already formed $\mathrm{Zn}$ dendrites in situ is demanded. Yang et al. [29] proposed that the large current density accelerated dendrite formation, while low current density had little effect on dendrite growth. Based on this finding, they offered an electro-healing strategy from the perspective of regulating the charge/discharge protocols. Specifically, they manipulated the anode current density to mediate $\mathrm{Zn}$ deposition behavior. The morphology of the anode surface gradually became smooth with the passivation of dendrites during the constant current discharge/charge at a low current density ( $\leq 1 \mathrm{~mA} \mathrm{~cm}^{-2}$ ) (Fig. 26a). Obviously, by actively eliminating dendrites, this self-healing strategy can largely extend battery lifespan.

There have been reports of changing the configuration of the battery, such as changing the orientation of the anode reaction interface which can change the direction of dendrite growth to prevent battery short circuit (Fig. 26b) [179]. Currently reported ZIBs usually contain thick anodes caused by excess $\mathrm{Zn}$ metal, which results in low energy density. Therefore, the "anode-free" configuration applied in lithium and sodium metal batteries was introduced into ZIBs and further optimized (Fig. 26c). It is coating the $\mathrm{Cu}$ current collector with a modified layer of carbon nano-disks without carbon nanoparticles as $\mathrm{Zn}$ nucleation sites. In the initial cycle, since there is no $\mathrm{Zn}$ metal on the anode side, it is necessary to charge the anode first. The $\mathrm{Zn}$ involved in the cycle on the anode comes entirely from the electrolyte and cathode, which means that the $\mathrm{Zn}$ on the anode is zero excess. Thus, ZIBs with high energy density can be obtained (Fig. 26d).
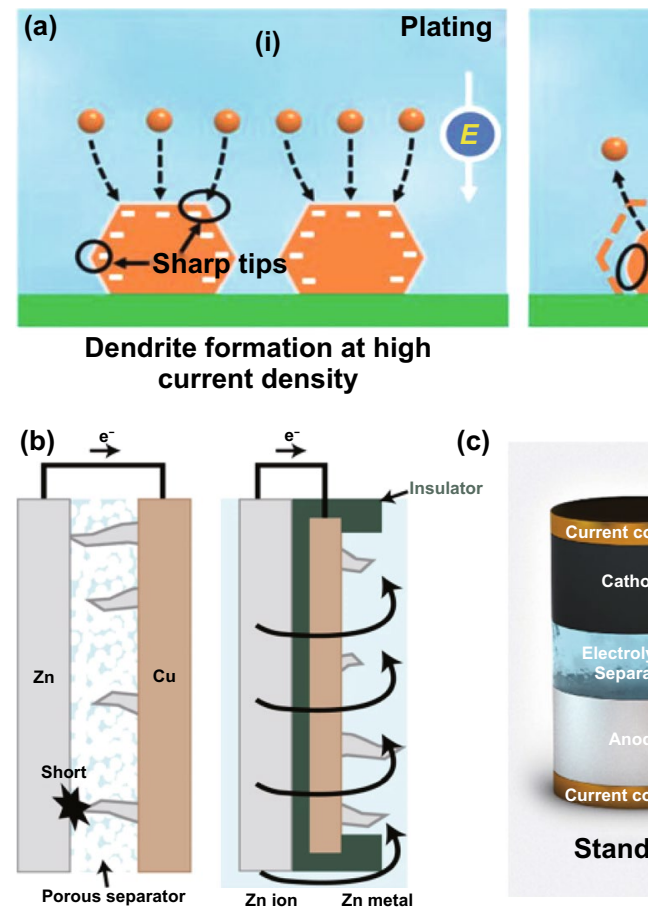

(c)

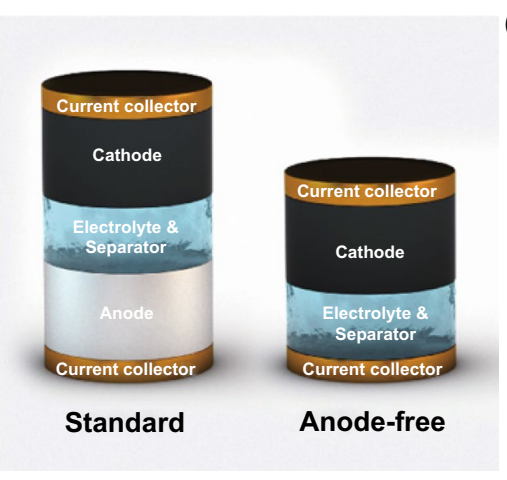

(ii) Stripping

Preferred stripping

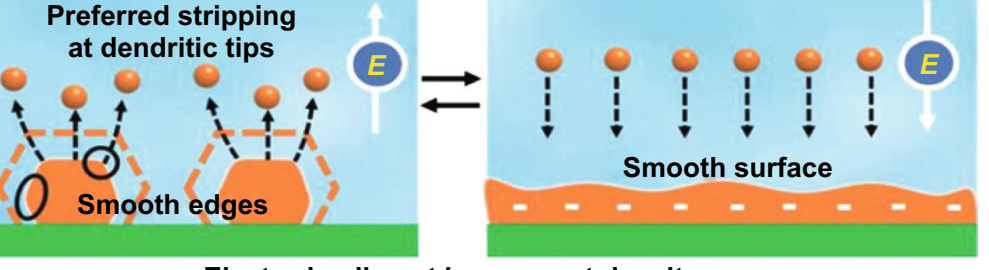

Electro-healing at low current density

$\mathrm{Zn}^{2+} \quad$ Plated Zn $\quad$ Zn electrode Electrolyte

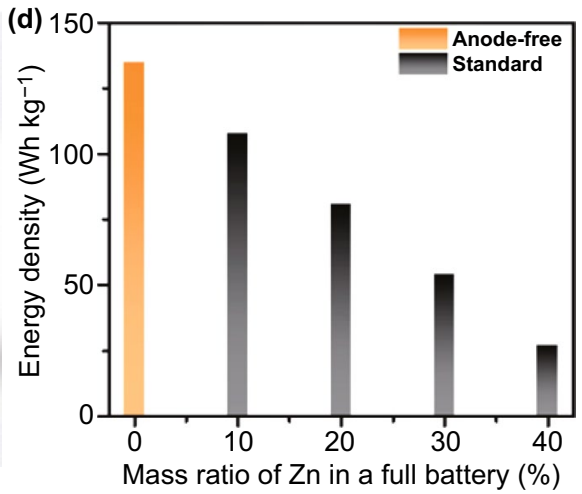

Fig. 26 a Schematic illustration of the electrohealing process, where the sharp tips of dendrites are passivated into smooth edges and finally produce a smooth electrode surface [29]. Copyright 2019, Wiley-VCH. b Schematic representation of (right) conventional frontside- and (left) backside-plating configuration cells [179]. $\mathbf{c}$ Schematic diagram of standard battery and anode-free battery; d Comparison of the energy density of the anode-free and standard batteries [180]. Copyright 2021, American Chemical Society 


\section{Summary and Perspectives}

Generally speaking, ZIBs have achieved rapid development due to their high specific capacity, safety, environmental friendliness, and low cost. As an efficient ESS, the aqueous ZIBs are expected to dominate the future energy storage market. Unfortunately, the problem of anode stability in mild aqueous ZIBs has not been well resolved. It has become an insurmountable stumbling block for the commercialization and large-scale application of mild aqueous ZIBs. Hence, in this review, three critical issues that plague the performance of mild aqueous ZIB anodes are analyzed in detail, including dendrite growth caused by the inherently limited diffusion of $\mathrm{Zn}^{2+}$ ions, hydrogen evolution, and corrosion induced by water splitting. Among them, dendrites and hydrogen evolutions are the most direct damage to battery performance and life, especially for those devices with large capacity. The anode interface is prone to rapid deterioration, thus resulting in battery short circuits or bulging. Different from the above two intuitive effects, the corrosion pits and by-products displayed at the corrosion site may not directly damage the battery but gradually reduce the battery performance since excessive $\mathrm{Zn}$ metal has a certain tolerance to anode corrosion. Importantly, these anode issues are closely interconnected and mutually reinforcing. Although there is a certain theoretical basis for the formation mechanism and influencing factors, the insightful understandings are far from enough, and controversies on some basic issues are still unable to reach consensus. For example, a large current density is more likely to cause $\mathrm{Zn}$ dendrites than a small one. Still, some researchers insist that a large current density is more conducive to dense $\mathrm{Zn}$ deposition and inhibits $\mathrm{Zn}$ dendrites. Subsequently, in the anode optimization part, the current relevant strategies to improve the anode performance were summarized combined with recent related reports. From the theoretical analysis perspective, each strategy's modification mechanism is explained in detail, involving interface modification, structural anode, alloying anode, intercalation anode, liquid electrolyte, non-liquid electrolyte, separator design, and other strategies. To better track the performance progress of ZIBs, the performance list of ZIBs related to $\mathrm{Zn}$ anode research are also summarized (Table $\mathrm{S} 1$ in the Supporting Information).

Through detailed analysis of the latest developments, it can be concluded that although the anode interface stability problem has made great progress, there is still much room for improvement. Therefore, some suggestions are proposed here:

(1) Deepen the research of fundamental mechanisms. Currently, the explanation of mechanisms that expound the influence of various strategies on the $\mathrm{Zn}$ deposition behavior in mild solutions is mainly based on ectopic analysis and theoretical simulations without enough convincing evidence. The interface reaction between the anode and the electrolyte remains ambiguous, and more attention is paid to the deposition process, but the stripping process is frequently ignored. Hence, it is necessary to conduct in-depth and systematic research on the $\mathrm{Zn}$ deposition and stripping mechanism in mild solutions from the molecular and atomic levels. Correspondingly, more comprehensive characterization techniques are required to explore the fundamental mechanisms, especially the direct observation that can obtain convincing first-hand data simply, precisely, and quickly. For example, in situ visualization characterization techniques (such as X-ray phase-contrast imaging, in situ SEM, in situ XRD, and in situ optical microscopy) are constantly evolving, which can be combined with phase-field simulation to analyze complex electrochemical behaviors. Also, significantly, the theoretical calculation (DFT calculation and other computational models) should be developed to predict and verify $\mathrm{Zn}$ deposition/stripping tendency on the anode and provide clear theoretical support for nucleation and growth at the interface.

(2) Formulate and improve quantify and test evaluation standards. Attributable to the failure of some critical parameters to be reasonably quantified, such as $\mathrm{Zn}$ reversibility. The current evaluation standard of anode reversibility is based on its lifespan and stability. Nevertheless, it ignores the irreversible process that excess $\mathrm{Zn}$ can make up for the capacity loss caused by $\mathrm{Zn}$ death or corrosion, which is at the cost of reducing the battery's energy density. Besides, most reports ignore the detailed quantification of $\mathrm{H}_{2}$ evolution regardless of mild electrolytes that are more likely to cause HER than alkaline electrolytes, which increases the difficulty of analyzing the mechanism of capacity loss and battery failure. Besides, almost all reports claimed that they had constructed a perfect $\mathrm{Zn}$ anode and obtained enhanced battery performance accordingly. But their results are based on different test protocols such as the amount of electrolyte, test device, test temperature, current density, and depth of discharge (DOD). These test conditions are challenging to be unified, so that 
the experimental data results lack comparability to a certain extent. Therefore, utilizing multiple methods to form and improve quantify and test evaluation standards is beneficial to developing $\mathrm{Zn}$ anodes.

(3) Combine multiple strategies. Given that the anode interface problems are interconnected, a combination of strategies to solve various issues simultaneously can make up for the limited improvement in the anode. For example, some electrolyte additives can not only coordinate with $\mathrm{Zn}^{2+}$ ions to destroy the solvation structure, but can also be adsorbed on the metal surface to shield the direct contact of water and limit $\mathrm{Zn}$ nucleation and growth area. Combining structured anodes with additives or introducing additives into the gel electrolyte can achieve a stable anode interface. In addition, by introducing metal or metal oxide particles into the modified layer or structured anode, the composite anode can be constructed to strengthen the regulation of $\mathrm{Zn}$ deposition.

(4) Develop multifunctional separators. Since separators are connected to all the components of ZIBs, suitable separators can benefit the performance of both cathode and anode at the same time. However, separators commonly used in laboratories (glass fiber or cellulose separators) are challenging to maintain the long-term stability of anodes because they have poor mechanical strength and uneven ion transport channels. At present, there is little research on separators. Given that anode and separator are closely connected in the battery configuration, some anode interface modification strategies can be applied to the separator, such as compounding separators with some organic molecules with polar groups to obtain zincophilicity or constructing negative surface charges to inhibit anion transport and guide uniform cation migration.

(5) Focus on comprehensive cost and benefits. Commercial batteries emphasize cost and energy density. Especially for the former, $\mathrm{Zn}$ content should be reasonably constrained to ensure good cycling stability. However, to deliberately pursue the excellent electrochemical performance of the battery, some aqueous ZIBs contain excessive $\mathrm{Zn}$ (most $\mathrm{Zn}$ does not participate in the electrochemical reaction) or use high-concentration electrolyte strategies, which will dramatically increase the cost and fundamentally reduce the battery energy density. Applying anodes with a high specific surface area, such as $\mathrm{Zn}$ powder, is a promising method to increase $\mathrm{Zn}$ utilization and $\mathrm{Zn}$ content. Moreover, compared with other energy storage devices, low cost, high safety, and environmental friendliness are the basic characteristics of aqueous $\mathrm{ZIBs,} \mathrm{essential} \mathrm{requirements} \mathrm{for}$ long-term development. But considerable research places too much emphasis on dendrite-free and side reaction anodes but ignores these factors. Instead, they adopt expensive and harmful raw materials or complex technical routes that are difficult to produce on a large scale. Based on this status quo, it is recommended that the cost and benefit are comprehensively evaluated in designing reversible $\mathrm{Zn}$ anodes.

Acknowledgements This work was supported by the National Natural Science Foundation of China (No. 52071171), Liaoning Revitalization Talents Program-Pan Deng Scholars (XLYC1802005), Liaoning BaiQianWan Talents Program (LNBQW2018B0048), Natural Science Fund of Liaoning Province for Excellent Young Scholars (2019-YQ-04), Key Project of Scientific Research of the Education Department of Liaoning Province (LZD201902), Foundation for Young Scholars of Liaoning University (a252102001), Australian Research Council (ARC) Future Fellowship (FT210100298), CSIRO Energy Centre, Kick-Start Project and the Victorian Government's support through the provision of a grant from veski-Study Melbourne Research Partnerships (SMRP) project.

Funding Open access funding provided by Shanghai Jiao Tong University.

Open Access This article is licensed under a Creative Commons Attribution 4.0 International License, which permits use, sharing, adaptation, distribution and reproduction in any medium or format, as long as you give appropriate credit to the original author(s) and the source, provide a link to the Creative Commons licence, and indicate if changes were made. The images or other third party material in this article are included in the article's Creative Commons licence, unless indicated otherwise in a credit line to the material. If material is not included in the article's Creative Commons licence and your intended use is not permitted by statutory regulation or exceeds the permitted use, you will need to obtain permission directly from the copyright holder. To view a copy of this licence, visit http://creativecommons.org/licenses/by/4.0/.

Supplementary Information The online version contains supplementary material available at https://doi.org/10.1007/ s40820-021-00782-5.

\section{References}

1. B. Obama, The irreversible momentum of clean energy. Science 355(6321), 126-129 (2017). https://doi.org/10.1126/ science.aam6284

2. P.E. Brockway, A. Owen, L.I. Brand-Correa, L. Hardt, Estimation of global final-stage energy-return-on-investment for fossil fuels with comparison to renewable energy sources. 
Nat. Energy 4, 612-621 (2019). https://doi.org/10.1038/ s41560-019-0425-z

3. D.J. Davidson, Exnovating for a renewable energy transition. Nat. Energy 4, 254-256 (2019). https://doi.org/10.1038/ s41560-019-0369-3

4. S. Carley, D.M. Konisky, The justice and equity implications of the clean energy transition. Nat. Energy 5, 569-577 (2020). https://doi.org/10.1038/s41560-020-0641-6

5. N. Kittner, F. Lill, D.M. Kammen, Energy storage deployment and innovation for the clean energy transition. Nat. Energy 2, 17125 (2017). https://doi.org/10.1038/nenergy. 2017.125

6. W.P. Schill, Electricity storage and the renewable energy transition. Joule 4(10), 2059-2064 (2020). https://doi.org/ 10.1016/j.joule.2020.07.022

7. P.J. Heptonstall, R.J.K. Gross, A systematic review of the costs and impacts of integrating variable renewables into power grids. Nat. Energy 6, 72-83 (2021). https://doi.org/ 10.1038/s41560-020-00695-4

8. B. Steffen, F. Egli, M. Pahle, T.S. Schmidt, Navigating the clean energy transition in the COVID-19 crisis. Joule 4(6), 1137-1141 (2020). https://doi.org/10.1016/j.joule.2020.04. 011

9. T.M. Gür, Review of electrical energy storage technologies, materials and systems: challenges and prospects for largescale grid storage. Energy Environ. Sci. 11(10), 2696-2767 (2018). https://doi.org/10.1039/c8ee01419a

10. Y. Zheng, Y. Yao, J. Ou, M. Li, D. Luo et al., A review of composite solid-state electrolytes for lithium batteries: fundamentals, key materials and advanced structures. Chem. Soc. Rev. 49(23), 8790-8839 (2020). https://doi.org/10. 1039/d0cs00305k

11. F. Wu, J. Maier, Y. Yu, Guidelines and trends for nextgeneration rechargeable lithium and lithium-ion batteries. Chem. Soc. Rev. 49(5), 1569-1614 (2020). https://doi.org/ $10.1039 / \mathrm{c} 7 \mathrm{cs} 00863 \mathrm{e}$

12. T.B. Schon, B.T. McAllister, P.F. Li, D.S. Seferos, The rise of organic electrode materials for energy storage. Chem. Soc. Rev. 45(22), 6345-6404 (2016). https://doi.org/10. 1039/c6cs00173d

13. Y. Chen, Y. Kang, Y. Zhao, L. Wang, J. Liu et al., A review of lithium-ion battery safety concerns: the issues, strategies, and testing standards. J. Energy Chem. 59, 83-99 (2021). https://doi.org/10.1016/j.jechem.2020.10.017

14. B. Liu, Y. Jia, C. Yuan, L. Wang, X. Gao et al., Safety issues and mechanisms of lithium-ion battery cell upon mechanical abusive loading: a review. Energy Storage Mater. 24, 85-112 (2020). https://doi.org/10.1016/j.ensm. 2019.06.036

15. H. Kim, J. Hong, K.Y. Park, H. Kim, S.W. Kim et al., Aqueous rechargeable $\mathrm{Li}$ and $\mathrm{Na}$ ion batteries. Chem. Rev. 114(23), 11788-11827 (2014). https://doi.org/10.1021/cr500 $232 \mathrm{y}$

16. H. Ao, Y. Zhao, J. Zhou, W. Cai, X. Zhang et al., Rechargeable aqueous hybrid ion batteries: developments and prospects.
J. Mater. Chem. A 7, 18708-18734 (2019). https://doi.org/10. 1039/c9ta06433h

17. D. Chao, W. Zhou, F. Xie, C. Ye, H. Li et al., Roadmap for advanced aqueous batteries: from design of materials to applications. Sci. Adv. (2020). https://doi.org/10.1126/sciadv. aba4098

18. J. Wang, Y. Yang, Y. Zhang, Y. Li, R. Sun et al., Strategies towards the challenges of zinc metal anode in rechargeable aqueous zinc ion batteries. Energy Stor. Mater. 35, 19-46 (2021). https://doi.org/10.1016/j.ensm.2020.10.027

19. C. Xu, B. Li, H. Du, F. Kang, Energetic zinc ion chemistry: the rechargeable zinc ion battery. Angew. Chem. Int. Ed. 51(4), 933-935 (2012). https://doi.org/10.1002/anie.20110 6307

20. Z. Yi, G. Chen, F. Hou, L. Wang, J. Liang, Strategies for the stabilization of $\mathrm{Zn}$ metal anodes for $\mathrm{Zn}$-ion batteries. Adv. Energy Mater. 11(1), 2003065 (2021). https://doi.org/ 10.1002/aenm.202003065

21. T. Yamamoto, T. Shoji, Rechargeable $\mathrm{Zn}|\mathrm{ZnSO} 4| \mathrm{MnO}_{2}-$ type cells. Inorganica Chim. Acta 117(2), L27-L28 (1986). https://doi.org/10.1016/S0020-1693(00)82175-1

22. Q. Li, X. Rui, D. Chen, Y. Feng, N. Xiao et al., A highcapacity ammonium vanadate cathode for zinc-ion battery. Nano-Micro Lett. 12, 67 (2020). https://doi.org/10.1007/ s40820-020-0401-y

23. S. Ding, M. Zhang, R. Qin, J. Fang, H. Ren et al., Oxygen-deficient $\beta-\mathrm{MnO}_{2} @$ graphene oxide cathode for high-rate and long-life aqueous zinc ion batteries. NanoMicro Lett. 13, 173 (2021). https://doi.org/10.1007/ s40820-021-00691-7

24. C. Han, W. Li, H.K. Liu, S. Dou, J. Wang, Principals and strategies for constructing a highly reversible zinc metal anode in aqueous batteries. Nano Energy 74, 104880 (2020). https://doi.org/10.1016/j.nanoen.2020.104880

25. C. Xie, Y. Li, Q. Wang, D. Sun, Y. Tang et al., Issues and solutions toward zinc anode in aqueous zinc-ion batteries: a mini review. Carbon Energy 2, 540-560 (2020). https://doi. org/10.1002/cey2.67

26. R. Zhang, X.R. Chen, X. Chen, X.B. Cheng, X.Q. Zhang et al., Lithiophilic sites in doped graphene guide uniform lithium nucleation for dendrite-free lithium metal anodes. Angew. Chem. Int. Ed. 129(27), 7872-7876 (2017). https:// doi.org/10.1002/ange.201702099

27. W. Du, E.H. Ang, Y. Yang, Y. Zhang, M. Ye et al., Challenges in the material and structural design of zinc anode towards high-performance aqueous zinc-ion batteries. Energy Environ. Sci. 13(10), 3330-3360 (2020). https://doi.org/10.1039/ d0ee02079f

28. Q. Zhang, J. Luan, Y. Tang, X. Ji, H. Wang, Interfacial design of dendrite-free zinc anodes for aqueous zinc-ion batteries. Angew. Chem. Int. Ed. 59(32), 13180-13191 (2020). https:// doi.org/10.1002/anie.202000162

29. Q. Yang, G. Liang, Y. Guo, Z. Liu, B. Yan et al., Do zinc dendrites exist in neutral zinc batteries: a developed electrohealing strategy to in situ rescue in-service batteries. Adv. Mater. 
31(43), 1903778 (2019). https://doi.org/10.1002/adma.20190 3778

30. R. Zhang, X.B. Cheng, C.Z. Zhao, H.J. Peng, J.L. Shi et al., Conductive nanostructured scaffolds render low local current density to inhibit lithium dendrite growth. Adv. Mater. 28(11), 2155-2162 (2016). https://doi.org/10.1002/adma. 201504117

31. Y. Liu, X. Xu, M. Sadd, O.O. Kapitanova, V.A. Krivchenko et al., Insight into the critical role of exchange current density on electrodeposition behavior of lithium metal. Adv. Sci. 8(5), 2003301 (2021). https://doi.org/10.1002/advs.20200 3301

32. Y. Zuo, K. Wang, P. Pei, M. Wei, X. Liu et al., Zinc dendrite growth and inhibition strategies. Mater. Today Energy 20, 100692 (2021). https://doi.org/10.1016/j.mtener.2021.100692

33. P. He, J. Huang, Detrimental effects of surface imperfections and unpolished edges on the cycling stability of a zinc foil anode. ACS Energy Lett. 6(5), 1990-1995 (2021). https://doi. org/10.1021/acsenergylett.1c00638

34. F. Xie, H. Li, X. Wang, X. Zhi, D. Chao et al., Mechanism for zincophilic sites on zinc-metal anode hosts in aqueous batteries. Adv. Energy Mater. 11, 2003419 (2021). https:// doi.org/10.1002/aenm.202003419

35. L.N. Bengoa, S. Bruno, H.A. Lazzarino, P.R. Seré, W.A. Egli, Study of dendritic growth of zinc crystals on the edges of steel sheet. J. Appl. Electrochem. 44, 1261-1269 (2014). https://doi.org/10.1007/s10800-014-0722-y

36. K. Yan, J. Wang, S. Zhao, D. Zhou, B. Sun et al., Temperature-dependent nucleation and growth of dendrite-free lithium metal anodes. Angew. Chem. Int. Ed. 131(33), 1148611490 (2019). https://doi.org/10.1002/ange.201905251

37. L.N. Bengoa, P. Pary, P.R. Seré, M.S. Conconi, W.A. Egli, Dendritic zinc growth in acid electrolyte: effect of the $\mathrm{pH}$. J. Mater. Eng. Perform. 27, 1103-1108 (2018). https://doi.org/ 10.1007/s11665-018-3139-7

38. M.K. Aslam, Y. Niu, T. Hussain, H. Tabassum, W. Tang et al., How to avoid dendrite formation in metal batteries: innovative strategies for dendrite suppression. Nano Energy 86, 106142 (2021). https://doi.org/10.1016/j.nanoen.2021. 106142

39. Z. Hong, Z. Ahmad, V. Viswanathan, Design principles for dendrite suppression with porous polymer/aqueous solution hybrid electrolyte for $\mathrm{Zn}$ metal anodes. ACS Energy Lett. 5(8), 2466-2474 (2020). https://doi.org/10.1021/acsenergyl ett.0c01235

40. J. Hao, X. Li, X. Zeng, D. Li, J. Mao et al., Deeply understanding the $\mathrm{Zn}$ anode behaviour and corresponding improvement strategies in different aqueous $\mathrm{Zn}$-based batteries. Energy Environ. Sci. 13(11), 3917-3949 (2020). https://doi. org/10.1039/d0ee02162h

41. J. Hao, X. Li, S. Zhang, F. Yang, X. Zeng et al., Designing dendrite-free zinc anodes for advanced aqueous zinc batteries. Adv. Funct. Mater. 30(30), 2001263 (2020). https://doi. org/10.1002/adfm.202001263

42. J. Zhao, J. Zhang, W. Yang, B. Chen, Z. Zhao et al., "Waterin-deep eutectic solvent" electrolytes enable zinc metal anodes for rechargeable aqueous batteries. Nano Energy $\mathbf{5 7}$, 625-634 (2019). https://doi.org/10.1016/j.nanoen.2018.12. 086

43. Z. Zhao, J. Zhao, Z. Hu, J. Li, J. Li et al., Long-life and deeply rechargeable aqueous $\mathrm{Zn}$ anodes enabled by a multifunctional brightener-inspired interphase. Energy Environ. Sci. 12(6), 1938-1949 (2019). https://doi.org/10.1039/c9ee00596j

44. X. Guo, Z. Zhang, J. Li, N. Luo, G.L. Chai et al., Alleviation of dendrite formation on zinc anodes via electrolyte additives. ACS Energy Lett. 6, 395-403 (2021). https://doi.org/10.1021/ acsenergylett.0c02371

45. C. Li, X. Xie, S. Liang, J. Zhou, Issues and future perspective on zinc metal anode for rechargeable aqueous zinc-ion batteries. Energy Environ. Mater. 3, 146-159 (2020). https:// doi.org/10.1002/eem2.12067

46. Z. Cao, P. Zhuang, X. Zhang, M. Ye, J. Shen et al., Strategies for dendrite-free anode in aqueous rechargeable zinc ion batteries. Adv. Energy Mater. 10, 2001599 (2020). https://doi. org/10.1002/aenm.202001599

47. A. Bayaguud, Y. Fu, C. Zhu, Interfacial parasitic reactions of zinc anodes in zinc ion batteries: underestimated corrosion and hydrogen evolution reactions and their suppression strategies. J. Energy Chem. 64, 246-262 (2022). https://doi. org/10.1016/j.jechem.2021.04.016

48. L. Ma, Q. Li, Y. Ying, F. Ma, S. Chen et al., Toward practical high-areal-capacity aqueous zinc-metal batteries: quantifying hydrogen evolution and a solid-ion conductor for stable zinc anodes. Adv. Mater. 33(12), 2007406 (2021). https://doi.org/ 10.1002/adma.202007406

49. M. Zhu, J. Hu, Q. Lu, H. Dong, D.D. Karnaushenko et al., A patternable and in situ formed polymeric zinc blanket for a reversible zinc anode in a skin-mountable microbattery. Adv. Mater. 33(8), 2007497 (2021). https://doi.org/10.1002/adma. 202007497

50. F. Xie, H. Li, X. Wang, X. Zhi, D. Chao et al., Mechanism for zincophilic sites on zinc-metal anode hosts in aqueous batteries. Adv. Energy Mater. 11(9), 2003419 (2021). https:// doi.org/10.1002/aenm.202003419

51. S. Zhang, J. Hao, D. Luo, P. Zhang, B. Zhang et al., Dualfunction electrolyte additive for highly reversible $\mathrm{Zn}$ anode. Adv. Energy Mater. 11(37), 2102010 (2021). https://doi.org/ 10.1002/aenm. 202102010

52. C. Liu, X. Xie, B. Lu, J. Zhou, S. Liang, Electrolyte strategies toward better zinc-ion batteries. ACS Energy Lett. 6(3), 1015-1033 (2021). https://doi.org/10.1021/acsenergylett. 0c02684

53. Z. Lu, Y. Wu, L. Ding, Y. Wei, H. Wang, A lamellar MXene $\left(\mathrm{Ti}_{3} \mathrm{C}_{2} \mathrm{~T}_{\mathrm{x}}\right) / \mathrm{PSS}$ composite membrane for fast and selective lithium-ion separation. Angew. Chem. Int. Ed. 133(41), 22439-22443 (2021). https://doi.org/10.1002/ange.20210 8801

54. Y. Cui, Q. Zhao, X. Wu, X. Chen, J. Yang et al., An interface-bridged organic-inorganic layer that suppresses dendrite formation and side reactions for ultra-long-life aqueous zinc metal anodes. Angew. Chem. Int. Ed. 59(38), 16594-16601 (2020). https://doi.org/10.1002/anie.202005472 
55. Q. Yang, Y. Guo, B. Yan, C. Wang, Z. Liu et al., Hydrogen-substituted graphdiyne ion tunnels directing concentration redistribution for commercial-grade dendrite-free zinc anodes. Adv. Mater. 32(25), 2001755 (2020). https://doi.org/ 10.1002/adma.202001755

56. L. Kang, M. Cui, F. Jiang, Y. Gao, H. Luo et al., Nanoporous $\mathrm{CaCO}_{3}$ coatings enabled uniform $\mathrm{Zn}$ stripping/plating for long-life zinc rechargeable aqueous batteries. Adv. Energy Mater. 8, 1801090 (2018). https://doi.org/10.1002/aenm. 201801090

57. C. Deng, X. Xie, J. Han, Y. Tang, J. Gao et al., A sievefunctional and uniform-porous kaolin layer toward stable zinc metal anode. Adv. Funct. Mater. 30(21), 2000599 (2020). https://doi.org/10.1002/adfm.202000599

58. L. Hong, X. Wu, C. Ma, W. Huang, Y. Zhou et al., Boosting the $\mathrm{Zn}$-ion transfer kinetics to stabilize the $\mathrm{Zn}$ metal interface for high-performance rechargeable $\mathrm{Zn}$-ion batteries. J. Mater. Chem. A 9(31), 16814-16823 (2021). https://doi.org/ 10.1039/d1ta03967a

59. H. Yan, S. Li, Y. Nan, S. Yang, B. Li, Ultrafast zinc-ion-conductor interface toward high-rate and stable zinc metal batteries. Adv. Energy Mater. 11(18), 2100186 (2021). https:// doi.org/10.1002/aenm.202100186

60. Y. Yang, C. Liu, Z. Lv, H. Yang, X. Cheng et al., Redistributing $\mathrm{Zn}$-ion flux by interlayer ion channels in $\mathrm{Mg}$-Al layered double hydroxide-based artificial solid electrolyte interface for ultra-stable and dendrite-free $\mathrm{Zn}$ metal anodes. Energy Stor. Mater. 41, 230-239 (2021). https://doi.org/10.1016/j. ensm.2021.06.002

61. Z. Li, W. Deng, C. Li, W. Wang, Z. Zhou et al., Uniformizing the electric field distribution and ion migration during zinc plating/stripping via a binary polymer blend artificial interphase. J. Mater. Chem. A 8(34), 17725-17731 (2020). https://doi.org/10.1039/d0ta05253a

62. Z. Cao, X. Zhu, D. Xu, P. Dong, M.O.L. Chee et al., Eliminating $\mathrm{Zn}$ dendrites by commercial cyanoacrylate adhesive for zinc ion battery. Energy Stor. Mater. 36, 132-138 (2021). https://doi.org/10.1016/j.ensm.2020.12.022

63. P. Chen, X. Yuan, Y. Xia, Y. Zhang, L. Fu et al., An artificial polyacrylonitrile coating layer confining zinc dendrite growth for highly reversible aqueous zinc-based batteries. Adv. Sci. 8(11), 2100309 (2021). https://doi.org/10.1002/advs.20210 0309

64. X. Zhang, J. Li, D. Liu, M. Liu, T. Zhou et al., Ultra-long-life and highly reversible $\mathrm{Zn}$ metal anodes enabled by a desolvation and deanionization interface layer. Energy Environ. Sci. 14(5), 3120-3129 (2021). https://doi.org/10.1039/D0EE0 3898A

65. L.T. Hieu, S. So, I.T. Kim, J. Hur, Zn anode with flexible $\beta$-PVDF coating for aqueous $\mathrm{Zn}$-ion batteries with long cycle life. Chem. Eng. J. 411, 128584 (2021). https://doi.org/10. 1016/j.cej.2021.128584

66. T. Zhang, Y. Tang, S. Guo, X. Cao, A. Pan et al., Fundamentals and perspectives in developing zinc-ion battery electrolytes: a comprehensive review. Energy Environ. Sci. 13(12), 4625-4665 (2020). https://doi.org/10.1039/D0EE02620D
67. Y. Yang, C. Liu, Z. Lv, H. Yang, Y. Zhang et al., Synergistic manipulation of $\mathrm{Zn}^{2+}$ ion flux and desolvation effect enabled by anodic growth of a $3 \mathrm{D} \mathrm{ZnF}_{2}$ matrix for long-lifespan and dendrite-free $\mathrm{Zn}$ metal anodes. Adv. Mater. 33(11), 2007388 (2021). https://doi.org/10.1002/adma.202007388

68. X. Xie, S. Liang, J. Gao, S. Guo, J. Guo et al., Manipulating the ion-transfer kinetics and interface stability for highperformance zinc metal anodes. Energy Environ. Sci. 13(2), 503-510 (2020). https://doi.org/10.1039/c9ee03545a

69. P. Cao, X. Zhou, A. Wei, Q. Meng, H. Ye et al., Fast-charging and ultrahigh-capacity zinc metal anode for high-performance aqueous zinc-ion batteries. Adv. Funct. Mater. 31(20), 2100398 (2021). https://doi.org/10.1002/adfm. 202100398

70. J. Hao, B. Li, X. Li, X. Zeng, S. Zhang et al., An in-depth study of $\mathrm{Zn}$ metal surface chemistry for advanced aqueous Zn-ion batteries. Adv. Mater. 32(34), e2003021 (2020). https://doi.org/10.1002/adma.202003021

71. H. Palneedi, M. Peddigari, G.T. Hwang, D.Y. Jeong, J. Ryu, High-performance dielectric ceramic films for energy storage capacitors: progress and outlook. Adv. Funct. Mater. 28(42), 1803665 (2018). https://doi.org/10.1002/adfm.201803665

72. M.H. Braga, J.E. Oliveira, T. Kai, A.J. Murchison, A.J. Bard et al., Extraordinary dielectric properties at heterojunctions of amorphous ferroelectrics. J. Am. Chem. Soc. 140(51), 17968-17976 (2018). https://doi.org/10.1021/jacs.8b09603

73. K. Wu, J. Yi, X. Liu, Y. Sun, J. Cui et al., Regulating Zn deposition via an artificial solid-electrolyte interface with aligned dipoles for long life $\mathrm{Zn}$ anode. Nano-Micro Lett. 13, 79 (2021). https://doi.org/10.1007/s40820-021-00599-2

74. P. Liang, J. Yi, X. Liu, K. Wu, Z. Wang et al., Highly reversible $\mathrm{Zn}$ anode enabled by controllable formation of nucleation sites for Zn-based batteries. Adv. Funct. Mater. 30(13), 1908528 (2020). https://doi.org/10.1002/adfm.201908528

75. Z. Zhou, Y. Zhang, P. Chen, Y. Wu, H. Yang et al., Graphene oxide-modified zinc anode for rechargeable aqueous batteries. Chem. Eng. Sci. 194, 142-147 (2019). https://doi.org/10. 1016/j.ces.2018.06.048

76. C. Shen, X. Li, N. Li, K. Xie, J. gan Wang et al., Grapheneboosted, high-performance aqueous $\mathrm{Zn}$-ion battery. ACS Appl. Mater. Interfaces 10(30), 25446-25453 (2018)

77. A. Xia, X. Pu, Y. Tao, H. Liu, Y. Wang, Graphene oxide spontaneous reduction and self-assembly on the zinc metal surface enabling a dendrite-free anode for long-life zinc rechargeable aqueous batteries. Appl. Surf. Sci. 481, 852-859 (2019). https://doi.org/10.1016/j.apsusc.2019.03.197

78. Z. Li, L. Wu, S. Dong, T. Xu, S. Li et al., Pencil drawing stable interface for reversible and durable aqueous zinc-ion batteries. Adv. Funct. Mater. 31(4), 2006495 (2021). https:// doi.org/10.1002/adfm.202006495

79. L. Dong, W. Yang, W. Yang, H. Tian, Y. Huang et al., Flexible and conductive scaffold-stabilized zinc metal anodes for ultralong-life zinc-ion batteries and zinc-ion hybrid capacitors. Chem. Eng. J. 384, 123355 (2020). https://doi.org/10. 1016/j.cej.2019.123355 
80. Y. Lee, B. Ma, P. Bai, Concentration polarization and metal dendrite initiation in isolated electrolyte microchannels. Energy Environ. Sci. 13(10), 3504-3513 (2020). https://doi. org/10.1039/D0EE01874K

81. N. Zhang, S. Huang, Z. Yuan, J. Zhu, Z. Zhao et al., Direct self-assembly of MXene on $\mathrm{Zn}$ anodes for dendrite-free aqueous zinc-ion batteries. Angew. Chem. Int. Ed. 60(6), 28612865 (2021). https://doi.org/10.1002/anie.202012322

82. R. Yuksel, O. Buyukcakir, W.K. Seong, R.S. Ruoff, Metalorganic framework integrated anodes for aqueous zinc-ion batteries. Adv. Energy Mater. 10, 1904215 (2020). https:// doi.org/10.1002/aenm.201904215

83. D. Chao, C.R. Zhu, M. Song, P. Liang, X. Zhang et al., A high-rate and stable quasi-solid-state zinc-ion battery with novel 2D layered zinc orthovanadate array. Adv. Mater. 30(32), 1803181 (2018). https://doi.org/10.1002/adma.20180 3181

84. M. Cui, Y. Xiao, L. Kang, W. Du, Y. Gao et al., Quasi-isolated $\mathrm{Au}$ particles as heterogeneous seeds to guide uniform $\mathrm{Zn}$ deposition for aqueous zinc-ion batteries. ACS Appl. Energy Mater. 2, 6490-6496 (2019). https://doi.org/10.1021/ acsaem.9b01063

85. X. Chen, X.R. Chen, T.Z. Hou, B.Q. Li, X.B. Cheng et al., Lithiophilicity chemistry of heteroatom-doped carbon to guide uniform lithium nucleation in lithium metal anodes. Sci. Adv. (2019). https://doi.org/10.1126/sciadv.aau7728

86. Q. Zhang, J. Luan, X. Huang, Q. Wang, D. Sun et al., Revealing the role of crystal orientation of protective layers for stable zinc anode. Nat. Commun. 11, 3961 (2020). https://doi. org/10.1038/s41467-020-17752-x

87. Y. Yin, S. Wang, Q. Zhang, Y. Song, N. Chang et al., Dendrite-free zinc deposition Induced by tin-modified multifunctional 3D host for stable zinc-based flow battery. Adv. Mater. 32(6), 1906803 (2020). https://doi.org/10.1002/adma.20190 6803

88. D. Han, S. Wu, S. Zhang, Y. Deng, C. Cui et al., A corrosion-resistant and dendrite-free zinc metal anode in aqueous systems. Small 16, 2001736 (2020). https://doi.org/10.1002/ smll.202001736

89. Y. Zhang, G. Wang, F. Yu, G. Xu, Z. Li et al., Highly reversible and dendrite-free $\mathrm{Zn}$ electrodeposition enabled by a thin metallic interfacial layer in aqueous batteries. Chem. Eng. J. 416, 128062 (2021). https://doi.org/10.1016/j.cej.2020. 128062

90. W. Guo, Y. Zhang, X. Tong, X. Wang, L. Zhang et al., Multifunctional tin layer enabled long-life and stable anode for aqueous zinc-ion batteries. Mater. Today Energy 20, 100675 (2021). https://doi.org/10.1016/j.mtener.2021.100675

91. S. Li, J. Fu, G. Miao, S. Wang, W. Zhao et al., Toward planar and dendrite-free $\mathrm{Zn}$ electrodepositions by regulating $\mathrm{Sn}$ crystal textured surface. Adv. Mater. 33(21), 2008424 (2021). https://doi.org/10.1002/adma.202008424

92. M. Zhou, S. Guo, J. Li, X. Luo, Z. Liu et al., Surface-preferred crystal plane for a stable and reversible zinc anode. Adv. Mater. 33(21), 2100187 (2021). https://doi.org/10.1002/ adma.202100187
93. X. Jia, C. Liu, Z.G. Neale, J. Yang, G. Cao, Active materials for aqueous zinc ion batteries: synthesis, crystal structure, morphology, and electrochemistry. Chem. Rev. 120(15), 7795-7866 (2020). https://doi.org/10.1021/acs.chemrev. $9 \mathrm{~b} 00628$

94. C. Li, X. Shi, S. Liang, X. Ma, M. Han et al., Spatially homogeneous copper foam as surface dendrite-free host for zinc metal anode. Chem. Eng. J. 379, 122248 (2020). https://doi. org/10.1016/j.cej.2019.122248

95. X. Shi, G. Xu, S. Liang, C. Li, S. Guo et al., Homogeneous deposition of zinc on three-dimensional porous copper foam as a superior zinc metal anode. ACS Sustain. Chem. Eng. 7(21), 17737-17746 (2019). https://doi.org/10.1021/acssu schemeng.9b04085

96. Z. Kang, C. Wu, L. Dong, W. Liu, J. Mou et al., 3D porous copper skeleton supported zinc anode toward high capacity and long cycle life zinc ion batteries. ACS Sustain. Chem. Eng. 7(3), 3364-3371 (2019). https://doi.org/10.1021/acssu schemeng.8b05568

97. Q. Zhang, J. Luan, X. Huang, L. Zhu, Y. Tang et al., Simultaneously regulating the ion distribution and electric field to achieve dendrite-free $\mathrm{Zn}$ anode. Small 16(35), 2000929 (2020). https://doi.org/10.1002/smll.202000929

98. G. Zhang, X. Zhang, H. Liu, J. Li, Y. Chen et al., 3D-printed multi-channel metal lattices enabling localized electric-field redistribution for dendrite-free aqueous $\mathrm{Zn}$ ion batteries. Adv. Energy Mater. 11(19), 2003927 (2021). https://doi.org/10. 1002/aenm.202003927

99. W. Guo, Z. Cong, Z. Guo, C. Chang, X. Liang et al., Dendrite-free $\mathrm{Zn}$ anode with dual channel $3 \mathrm{D}$ porous frameworks for rechargeable Zn batteries. Energy Stor. Mater. 30, 104112 (2020). https://doi.org/10.1016/j.ensm.2020.04.038

100. R. Xiao, Z. Cai, R. Zhan, J. Wang, Y. Ou et al., Localizing concentrated electrolyte in pore geometry for highly reversible aqueous Zn metal batteries. Chem. Eng. J. 420, 129642 (2021). https://doi.org/10.1016/j.cej.2021.129642

101. J. Wang, Z. Cai, R. Xiao, Y. Ou, R. Zhan et al., A chemically polished zinc metal electrode with a ridge-like structure for cycle-stable aqueous batteries. ACS Appl. Mater. Interfaces 12(20), 23028-23034 (2020). https://doi.org/10.1021/acsami. 0c05661

102. S. Zhai, N. Wang, X. Tan, K. Jiang, Z. Quan et al., Interfaceengineered dendrite-free anode and ultraconductive cathode for durable and high-rate fiber $\mathrm{Zn}$ dual-ion microbattery. Adv. Funct. Mater. 31(13), 2008894 (2021). https://doi.org/ 10.1002/adfm.202008894

103. H. Li, Z. Liu, G. Liang, Y. Huang, Y. Huang et al., Waterproof and tailorable elastic rechargeable yarn zinc ion batteries by a cross-linked polyacrylamide electrolyte. ACS Nano 12, 3140-3148 (2018). https://doi.org/10.1021/acsna no. 7 b09003

104. T. Chen, Y. Wang, Y. Yang, F. Huang, M. Zhu et al., Heterometallic seed-mediated zinc deposition on inkjet printed silver nanoparticles toward foldable and heat-resistant zinc batteries. Adv. Funct. Mater. 31(24), 2101607 (2021). https:// doi.org/10.1002/adfm.202101607 
105. L.P. Wang, N.W. Li, T.S. Wang, Y.X. Yin, Y.G. Guo et al., Conductive graphite fiber as a stable host for zinc metal anodes. Electrochim. Acta 244, 172-177 (2017). https://doi. org/10.1016/j.electacta.2017.05.072

106. Y. Zeng, X. Zhang, R. Qin, X. Liu, P. Fang et al., Dendritefree zinc deposition induced by multifunctional CNT frameworks for stable flexible $\mathrm{Zn}$-ion batteries. Adv. Mater. 31, 1903675 (2019). https://doi.org/10.1002/adma.201903675

107. Y. Du, X. Chi, J. Huang, Q. Qiu, Y. Liu, Long lifespan and high-rate $\mathrm{Zn}$ anode boosted by 3D porous structure and conducting network. J. Power Sour. 479, 228808 (2020). https:// doi.org/10.1016/j.jpowsour.2020.228808

108. Q. Li, Y. Wang, F. Mo, D. Wang, G. Liang et al., Calendar life of $\mathrm{Zn}$ batteries based on $\mathrm{Zn}$ anode with $\mathrm{Zn}$ powder/current collector structure. Adv. Energy Mater. 11(14), 2003931 (2021). https://doi.org/10.1002/aenm.202003931

109. J. Zheng, Q. Zhao, T. Tang, J. Yin, C.D. Quilty et al., Reversible epitaxial electrodeposition of metals in battery anodes. Science 366, 645-648 (2019). https://doi.org/10.1126/scien ce.aax6873

110. C. Lu, H. Zhou, Is the electrodeposition of metals really epitaxial in battery anodes? Sci. Bull. 65(18), 1524-1526 (2020). https://doi.org/10.1016/j.scib.2020.05.028

111. Z. Wang, J. Huang, Z. Guo, X. Dong, Y. Liu et al., A metalorganic framework host for highly reversible dendrite-free zinc metal anodes. Joule 3(5), 1289-1300 (2019). https://doi. org/10.1016/j.joule.2019.02.012

112. Y. Tian, Y. An, C. Wei, B. Xi, S. Xiong et al., Flexible and free-standing $\mathrm{Ti}_{3} \mathrm{C}_{2} \mathrm{~T}_{\mathrm{x}}$ MXene@Zn paper for dendrite-free aqueous zinc metal batteries and nonaqueous Lithium metal batteries. ACS Nano 13(10), 11676-11685 (2019). https:// doi.org/10.1021/acsnano.9b05599

113. C. Wei, Y. Tao, Y. An, Y. Tian, Y. Zhang et al., Recent advances of emerging 2D MXene for stable and dendrite-free metal anodes. Adv. Funct. Mater. 30(45), 2004613 (2020). https://doi.org/10.1002/adfm.202004613

114. J. Gu, Q. Zhu, Y. Shi, H. Chen, D. Zhang et al., Single zinc atoms immobilized on MXene $\left(\mathrm{Ti}_{3} \mathrm{C}_{2} \mathrm{Cl}_{\mathrm{x}}\right)$ layers toward dendrite-free lithium metal anodes. ACS Nano 14(1), 891-898 (2020). https://doi.org/10.1021/acsnano.9b08141

115. Y. Tian, Y. An, C. Liu, S. Xiong, J. Feng et al., Reversible zinc-based anodes enabled by zincophilic antimony engineered MXene for stable and dendrite-free aqueous zinc batteries. Energy Stor. Mater. 41, 343-353 (2021). https://doi. org/10.1016/j.ensm.2021.06.019

116. C. Liu, Z. Luo, W. Deng, W. Wei, L. Chen et al., Liquid alloy interlayer for aqueous zinc-ion battery. ACS Energy Lett. 6(2), 675-683 (2021). https://doi.org/10.1021/acsenergylett. 0c02569

117. H. Jia, Z. Wang, M. Dirican, S. Qiu, C.Y. Chan et al., A liquid metal assisted dendrite-free anode for high-performance $\mathrm{Zn}$ ion batteries. J. Mater. Chem. A 9, 5597-5605 (2021). https:// doi.org/10.1039/d0ta11828a

118. X. Fan, H. Yang, X. Wang, J. Han, Y. Wu et al., Enabling stable $\mathrm{Zn}$ anode via a facile alloying strategy and 3D foam structure. Adv. Mater. Interfaces 8(7), 2002184 (2021). https://doi.org/10.1002/admi.202002184

119. Z. Cai, Y. Ou, J. Wang, R. Xiao, L. Fu et al., Chemically resistant $\mathrm{Cu}-\mathrm{Zn} / \mathrm{Zn}$ composite anode for long cycling aqueous batteries. Energy Stor. Mater. 27, 205-211 (2020). https:// doi.org/10.1016/j.ensm.2020.01.032

120. M. Fayette, H.J. Chang, I.A. Rodríguez-Pérez, X. Li, D. Reed, Electrodeposited zinc-based films as anodes for aqueous zinc batteries. ACS Appl. Mater. Interfaces 12(38), 42763-42772 (2020). https://doi.org/10.1021/acsami.0c10956

121. Y. Zhang, J.D. Howe, S. Ben-Yoseph, Y. Wu, N. Liu, Unveiling the origin of alloy-seeded and nondendritic growth of $\mathrm{Zn}$ for rechargeable aqueous Zn batteries. ACS Energy Lett. 6(2), 404-412 (2021). https://doi.org/10.1021/acsenergylett.0c023 43

122. Y. Wang, Y. Chen, W. Liu, X. Ni, P. Qing et al., Uniform and dendrite-free zinc deposition enabled by in situ formed $\mathrm{AgZn}_{3}$ for the zinc metal anode. J. Mater. Chem. A 9(13), 8452-8461 (2021). https://doi.org/10.1039/D0TA12177K

123. Q. Lu, C. Liu, Y. Du, X. Wang, L. Ding et al., Uniform $\mathrm{Zn}$ deposition achieved by Ag coating for improved aqueous zinc-ion batteries. ACS Appl. Mater. Interfaces 13(14), 16869-16875 (2021). https://doi.org/10.1021/acsami.0c229 11

124. S.B. Wang, Q. Ran, R.Q. Yao, H. Shi, Z. Wen et al., Lamellananostructured eutectic zinc-aluminum alloys as reversible and dendrite-free anodes for aqueous rechargeable batteries. Nat. Commun. 11, 1634 (2020). https://doi.org/10.1038/ s41467-020-15478-4

125. H. Tian, Z. Li, G. Feng, Z. Yang, D. Fox et al., Stable, highperformance, dendrite-free, seawater-based aqueous batteries. Nat. Commun. 12, 237 (2021). https://doi.org/10.1038/ s41467-020-20334-6

126. T.C. Li, D. Fang, J. Zhang, M.E. Pam, Z.Y. Leong et al., Recent progress in aqueous zinc-ion batteries: a deep insight into zinc metal anodes. J. Mater. Chem. A 9(10), 6013-6028 (2021). https://doi.org/10.1039/d0ta09111a

127. W.J. Zhang, Lithium insertion/extraction mechanism in alloy anodes for lithium-ion batteries. J. Power Sour. 196(3), 877885 (2011). https://doi.org/10.1016/j.jpowsour.2010.08.114

128. S. Jin, Y. Ye, Y. Niu, Y. Xu, H. Jin et al., Solid-solution-based metal alloy phase for highly reversible lithium metal anode. J. Am. Chem. Soc. 142(19), 8818-8826 (2020). https://doi. org/10.1021/jacs.0c01811

129. T. Wang, C. Li, X. Xie, B. Lu, Z. He et al., Anode materials for aqueous zinc ion batteries: mechanisms, properties, and perspectives. ACS Nano 14(12), 16321-16347 (2020). https://doi.org/10.1021/acsnano.0c07041

130. Y. Tian, Y. An, C. Wei, B. Xi, S. Xiong et al., Recent advances and perspectives of Zn-metal free "rocking-chair"type Zn-ion batteries. Adv. Energy Mater. 11(5), 2002529 (2021). https://doi.org/10.1002/aenm.202002529

131. N. Liu, X. Wu, Y. Zhang, Y. Yin, C. Sun et al., Building high rate capability and ultrastable dendrite-free organic anode for 
rechargeable aqueous zinc batteries. Adv. Sci. 7(14), 2000146 (2020). https://doi.org/10.1002/advs.202000146

132. Y. Yang, J. Xiao, J. Cai, G. Wang, W. Du et al., Mixedvalence copper selenide as an anode for ultralong lifespan rocking-chair $\mathrm{Zn}$-ion batteries: an insight into its intercalation/extraction kinetics and charge storage mechanism. Adv. Funct. Mater. 31(3), 2005092 (2021). https://doi.org/10.1002/ adfm.202005092

133. N. Zhang, F. Cheng, Y. Liu, Q. Zhao, K. Lei et al., Cationdeficient spinel $\mathrm{ZnMn}_{2} \mathrm{O}_{4}$ cathode in $\mathrm{Zn}\left(\mathrm{CF}_{3} \mathrm{SO}_{3}\right)_{2}$ electrolyte for rechargeable aqueous $\mathrm{Zn}$-ion battery. J. Am. Chem. Soc. 138(39), 12894-12901 (2016). https://doi.org/10.1021/jacs. $6 \mathrm{~b} 05958$

134. F. Wang, O. Borodin, T. Gao, X. Fan, W. Sun et al., Highly reversible zinc metal anode for aqueous batteries. Nat. Mater. 17, 543-549 (2018). https://doi.org/10.1038/ s41563-018-0063-Z

135. C. Zhang, J. Holoubek, X. Wu, A. Daniyar, L. Zhu et al., A $\mathrm{ZnCl}_{2}$ water-in-salt electrolyte for a reversible $\mathrm{Zn}$ metal anode. Chem. Commun. 54(100), 14097-14099 (2018). https://doi.org/10.1039/c8cc07730d

136. P. Sun, L. Ma, W. Zhou, M. Qiu, Z. Wang et al., Simultaneous regulation on solvation shell and electrode interface for dendrite-free $\mathrm{Zn}$ ion batteries achieved by a low-cost glucose additive. Angew. Chem. Int. Ed. 60(33), 18247-18255 (2021). https://doi.org/10.1002/anie.202105756

137. Y. Zhang, M. Zhu, K. Wu, F. Yu, G. Wang et al., An indepth insight of a highly reversible and dendrite-free $\mathrm{Zn}$ metal anode in an hybrid electrolyte. J. Mater. Chem. A 9, 4253-4261 (2021). https://doi.org/10.1039/D0TA11668H

138. Z. Hou, H. Tan, Y. Gao, M. Li, Z. Lu et al., Tailoring desolvation kinetics enables stable zinc metal anodes. J. Mater. Chem. A 8(37), 19367-19374 (2020). https://doi.org/10. 1039/d0ta06622b

139. R. Qin, Y. Wang, M. Zhang, Y. Wang, S. Ding et al., Tuning $\mathrm{Zn}^{2+}$ coordination environment to suppress dendrite formation for high-performance Zn-ion batteries. Nano Energy 80, 105478 (2021). https://doi.org/10.1016/j.nanoen.2020. 105478

140. J. Hao, L. Yuan, C. Ye, D. Chao, K. Davey et al., Boosting zinc electrode reversibility in aqueous electrolytes by using low-cost antisolvents. Angew. Chem. Int. Ed. 60(13), 73667375 (2021). https://doi.org/10.1002/anie.202016531

141. A. Mitha, A.Z. Yazdi, M. Ahmed, P. Chen, Surface adsorption of polyethylene glycol to suppress dendrite formation on zinc anodes in rechargeable aqueous batteries. Chem. ElectroChem. 5, 2409-2418 (2018). https://doi.org/10.1002/celc. 201800572

142. Y. Jin, K.S. Han, Y. Shao, M.L. Sushko, J. Xiao et al., Stabilizing zinc anode reactions by polyethylene oxide polymer in mild aqueous electrolytes. Adv. Funct. Mater. 30(43), 2003932 (2020). https://doi.org/10.1002/adfm.202003932

143. M. Yan, C. Xu, Y. Sun, H. Pan, H. Li, Manipulating Zn anode reactions through salt anion involving hydrogen bonding network in aqueous electrolytes with PEO additive. Nano Energy
82, 105739 (2021). https://doi.org/10.1016/j.nanoen.2020. 105739

144. C. Sun, C. Wu, X. Gu, C. Wang, Q. Wang, Interface engineering via $\mathrm{Ti}_{3} \mathrm{C}_{2} \mathrm{~T}_{\mathrm{x}}$ MXene electrolyte additive toward dendritefree zinc deposition. Nano-Micro Lett. 13, 89 (2021). https:// doi.org/10.1007/s40820-021-00612-8

145. H. Lu, X. Zhang, M. Luo, K. Cao, Y. Lu et al., Amino acidinduced interface charge engineering enables highly reversible $\mathrm{Zn}$ anode. Adv. Funct. Mater. 31(45), 2103514 (2021). https://doi.org/10.1002/adfm.202103514

146. F. Ding, W. Xu, G.L. Graff, J. Zhang, M.L. Sushko et al., Dendrite-free lithium deposition via self-healing electrostatic shield mechanism. J. Am. Chem. Soc. 135(11), 4450-4456 (2013). https://doi.org/10.1021/ja312241y

147. S. Guo, L. Qin, T. Zhang, M. Zhou, J. Zhou et al., Fundamentals and perspectives of electrolyte additives for aqueous zinc-ion batteries. Energy Stor. Mater. 34, 545-562 (2021). https://doi.org/10.1016/j.ensm.2020.10.019

148. A. Bayaguud, X. Luo, Y. Fu, C. Zhu, Cationic surfactant-type electrolyte additive enables three-dimensional dendrite-free zinc anode for stable zinc-ion batteries. ACS Energy Lett. 5(9), 3012-3020 (2020). https://doi.org/10.1021/acsenergyl ett.0c01792

149. F. Wan, L. Zhang, X. Dai, X. Wang, Z. Niu et al., Aqueous rechargeable zinc/sodium vanadate batteries with enhanced performance from simultaneous insertion of dual carriers. Nat. Commun. 9, 1656 (2018). https://doi.org/10.1038/ s41467-018-04060-8

150. W. Xu, K. Zhao, W. Huo, Y. Wang, G. Yao et al., Diethyl ether as self-healing electrolyte additive enabled long-life rechargeable aqueous zinc ion batteries. Nano Energy 62, 275-281 (2019). https://doi.org/10.1016/j.nanoen.2019.05. 042

151. D. Li, L. Cao, T. Deng, S. Liu, C. Wang, Design of a solid electrolyte interphase for aqueous $\mathrm{Zn}$ batteries. Angew. Chem. Int. Ed. 60(23), 13035-13041 (2021). https://doi.org/ 10.1002/anie.202103390

152. Y. An, Y. Tian, K. Zhang, Y. Liu, C. Liu et al., Stable aqueous anode-free zinc batteries enabled by interfacial engineering. Adv. Funct. Mater. 31(26), 2101886 (2021). https://doi.org/ 10.1002/adfm.202101886

153. Y. Chu, S. Zhang, S. Wu, Z. Hu, G. Cui et al., In situ built interphase with high interface energy and fast kinetics for high performance Zn metal anodes. Energy Environ. Sci. 14(6), 3609-3620 (2021). https://doi.org/10.1039/D1EE00308A

154. L. Cao, D. Li, T. Pollard, T. Deng, B. Zhang et al., Fluorinated interphase enables reversible aqueous zinc battery chemistries. Nat. Nanotechnol. 16, 902-910 (2021). https:// doi.org/10.1038/s41565-021-00905-4

155. X. Zeng, J. Mao, J. Hao, J. Liu, S. Liu et al., Electrolyte design for in situ construction of highly $\mathrm{Zn}^{2+}$-conductive solid electrolyte interphase to enable high-performance aqueous $\mathrm{Zn}$ ion batteries under practical conditions. Adv. Mater. 33(11), 2007416 (2021). https://doi.org/10.1002/adma.202007416

156. L. Cao, D. Li, F.A. Soto, V. Ponce, B. Zhang et al., Highly reversible aqueous zinc batteries enabled by 
zincophilic-zincophobic interfacial layers and interrupted hydrogen-bond electrolytes. Angew. Chem. Int. Ed. 60(34), 18845-18851 (2021). https://doi.org/10.1002/anie.20210 7378

157. L. Ma, S. Chen, N. Li, Z. Liu, Z. Tang et al., Hydrogen-free and sendrite-free All-solid-state Zn-ion batteries. Adv. Mater. 32(14), 1908121 (2020). https://doi.org/10.1002/adma.201908121

158. Y. Cui, Q. Zhao, X. Wu, Z. Wang, R. Qin et al., Quasi-solid single $\mathrm{Zn}$-ion conductor with high conductivity enabling dendrite-free Zn metal anode. Energy Stor. Mater. 27, 1-8 (2020). https://doi.org/10.1016/j.ensm.2020.01.003

159. Z. Wang, J. Hu, L. Han, Z. Wang, H. Wang et al., A MOFbased single-ion $\mathrm{Zn}^{2+}$ solid electrolyte leading to dendritefree rechargeable Zn batteries. Nano Energy 56, 92-99 (2019). https://doi.org/10.1016/j.nanoen.2018.11.038

160. H. Dong, J. Li, J. Guo, F. Lai, F. Zhao et al., Insights on flexible zinc-ion batteries from lab research to commercialization. Adv. Mater. 33(20), 2007548 (2021). https://doi.org/10. 1002/adma.202007548

161. J. Huang, X. Chi, Y. Du, Q. Qiu, Y. Liu, Ultrastable zinc anodes enabled by anti-dehydration ionic liquid polymer electrolyte for aqueous $\mathrm{Zn}$ batteries. ACS Appl. Mater. Interfaces 13(3), 4008-4016 (2021). https://doi.org/10.1021/acsami. 0c20241

162. Z. Chen, X. Li, D. Wang, Q. Yang, L. Ma et al., Grafted MXene/polymer electrolyte for high performance solid zinc batteries with enhanced shelf life at low/high temperatures. Energy Environ. Sci. 14, 3492-3501 (2021). https://doi.org/ 10.1039/D1EE00409C

163. S. Huang, J. Zhu, J. Tian, Z. Niu, Recent progress in the electrolytes of aqueous zinc-ion batteries. Chem. A Eur. J. 25(64), 14480-14494 (2019). https://doi.org/10.1002/chem. 201902660

164. D. Lee, H.I. Kim, W.Y. Kim, S.K. Cho, K. Baek et al., Waterrepellent ionic liquid skinny gels customized for aqueous $\mathrm{Zn}$-ion battery anodes. Adv. Funct. Mater. 31(36), 2103850 (2021). https://doi.org/10.1002/adfm.202103850

165. J. Cong, X. Shen, Z. Wen, X. Wang, L. Peng et al., Ultrastable and highly reversible aqueous zinc metal anodes with high preferred orientation deposition achieved by a polyanionic hydrogel electrolyte. Energy Stor. Mater. 35, 586-594 (2021). https://doi.org/10.1016/j.ensm.2020.11.041

166. Y. Tang, C. Liu, H. Zhu, X. Xie, J. Gao et al., Ion-confinement effect enabled by gel electrolyte for highly reversible dendrite-free zinc metal anode. Energy Stor. Mater. 27, 109116 (2020). https://doi.org/10.1016/j.ensm.2020.01.023

167. Q. Han, X. Chi, Y. Liu, L. Wang, Y. Du et al., An inorganic salt reinforced $\mathrm{Zn}^{2+}$-conducting solid-state electrolyte for ultra-stable Zn metal batteries. J. Mater. Chem. A 7(39), 22287-22295 (2019). https://doi.org/10.1039/c9ta07218g

168. M. Chen, W. Zhou, A. Wang, A. Huang, J. Chen et al., Antifreezing flexible aqueous $\mathrm{Zn}-\mathrm{MnO}_{2}$ batteries working at -35 ${ }^{\circ} \mathrm{C}$ enabled by a borax-crosslinked polyvinyl alcohol/glycerol gel electrolyte. J. Mater. Chem. A 8(14), 6828-6841 (2020). https://doi.org/10.1039/d0ta01553a

169. M. Chen, J. Chen, W. Zhou, X. Han, Y. Yao et al., Realizing an all-round hydrogel electrolyte toward environmentally adaptive dendrite-free aqueous $\mathrm{Zn}-\mathrm{MnO}_{2}$ batteries. Adv. Mater. 33(9), 2007559 (2021). https://doi.org/10.1002/adma. 202007559

170. J. Liu, J. Long, Z. Shen, X. Jin, T. Han et al., A self-healing flexible quasi-solid zinc-ion battery using all-in-one electrodes. Adv. Sci. 8(8), 2004689 (2021). https://doi.org/10. 1002/advs.202004689

171. W. Deng, Z. Zhou, Y. Li, M. Zhang, X. Yuan et al., Highcapacity layered magnesium vanadate with concentrated gel electrolyte toward high-performance and wide-temperature zinc-ion battery. ACS Nano 14(11), 15776-15785 (2020). https://doi.org/10.1021/acsnano.0c06834

172. J. Gao, X. Xie, S. Liang, B. Lu, J. Zhou, Inorganic colloidal electrolyte for highly robust zinc-ion batteries. Nano-Micro Lett. 13, 69 (2021). https://doi.org/10.1007/ s40820-021-00595-6

173. B. Wu, Y. Wu, Z. Lu, J. Zhang, N. Han et al., A cation selective separator induced cathode protective layer and regulated zinc deposition for zinc ion batteries. J. Mater. Chem. A 9(8), 4734-4743 (2021). https://doi.org/10.1039/d0ta11841a

174. C. Li, Z. Sun, T. Yang, L. Yu, N. Wei et al., Directly grown vertical graphene carpets as janus separators toward stabilized Zn metal anodes. Adv. Mater. 32(33), 2003425 (2020). https://doi.org/10.1002/adma.202003425

175. Z. Wang, L. Dong, W. Huang, H. Jia, Q. Zhao et al., Simultaneously regulating uniform $\mathrm{Zn}^{2+}$ flux and electron conduction by MOF/rGO interlayers for high-performance $\mathrm{Zn}$ anodes. Nano-Micro Lett. 13, 73 (2021). https://doi.org/10.1007/ s40820-021-00594-7

176. Z. Hou, Y. Gao, H. Tan, B. Zhang, Realizing high-power and high-capacity zinc/sodium metal anodes through interfacial chemistry regulation. Nat. Commun. 12, 3083 (2021). https:// doi.org/10.1038/s41467-021-23352-0

177. J. Cao, D. Zhang, C. Gu, X. Zhang, M. Okhawilai et al., Modulating $\mathrm{Zn}$ deposition via ceramic-cellulose separator with interfacial polarization effect for durable zinc anode. Nano Energy 89, 106322 (2021). https://doi.org/10.1016/j.nanoen. 2021.106322

178. J. Cao, D. Zhang, C. Gu, X. Wang, S. Wang et al., Manipulating crystallographic orientation of zinc deposition for dendrite-free zinc ion batteries. Adv. Energy Mater. 11(29), 2101299 (2021). https://doi.org/10.1002/aenm.202101299

179. S. Higashi, S.W. Lee, J.S. Lee, K. Takechi, Y. Cui, Avoiding short circuits from zinc metal dendrites in anode by backsideplating configuration. Nat. Commun. 7, 11801 (2016). https:// doi.org/10.1038/ncomms 11801

180. Y. Zhu, Y. Cui, H.N. Alshareef, An anode-free $\mathrm{Zn}-\mathrm{MnO}_{2}$ battery. Nano Lett. 21(3), 1446-1453 (2021). https://doi.org/ 10.1021/acs.nanolett.0c04519 